\title{
Cancellous bone and theropod dinosaur locomotion. Part III - Inferring posture and locomotor biomechanics in extinct theropods, and its evolution on the line to birds
}

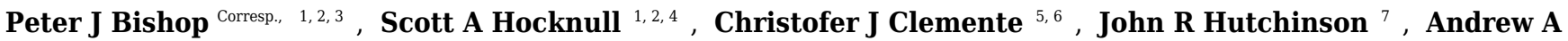 \\ Farke $^{8}$, Rod S Barrett ${ }^{2,3}$, David G Lloyd ${ }^{2,3}$ \\ ${ }^{1}$ Geosciences Program, Queensland Museum, Brisbane, Queensland, Australia \\ 2 School of Allied Health Sciences, Griffith University, Gold Coast, Queensland, Australia \\ ${ }^{3}$ Gold Coast Orthopaedic Research, Engineering and Education Alliance, Menzies Health Institute Queensland, Australia \\ 4 School of Biosciences, University of Melbourne, Melbourne, Victoria, Australia \\ 5 School of Science and Engineering, University of the Sunshine Coast, Maroochydore, Queensland, Australia \\ 6 School of Biological Sciences, University of Queensland, Brisbane, Queensland, Australia \\ 7 Structure and Motion Laboratory, Department of Comparative Biomedical Sciences, Royal Veterinary College, Hatfield, Hertfordshire, United Kingdom \\ 8 Raymond M. Alf Museum of Paleontology at The Webb Schools, Claremont, California, United States of America
}

Corresponding Author: Peter J Bishop

Email address: peter.bishop@qm.qld.gov.au

This paper is the last of a three-part series that investigates the architecture of cancellous bone in the main hindlimb bones of theropod dinosaurs, and uses cancellous bone architectural patterns to infer locomotor biomechanics in extinct non-avian species. Cancellous bone is highly sensitive to its prevailing mechanical environment, and may therefore help further understanding of locomotor biomechanics in extinct tetrapod vertebrates such as dinosaurs. Here in Part III, the biomechanical modelling approach derived previously was applied to two species of extinct, non-avian theropods, Daspletosaurus torosus and Troodon formosus . Observed cancellous bone architectural patterns were linked with quasi-static, three-dimensional musculoskeletal and finite element models of the hindlimb of both species, and used to derive characteristic postures that best aligned continuum-level principal stresses with cancellous bone fabric. The posture identified for Daspletosaurus was largely upright, with a subvertical femoral orientation, whilst that identified for Troodon was more crouched, but not to the degree observed in extant birds. In addition to providing new insight on posture and limb articulation, this study also tested previous hypotheses of limb bone loading mechanics and muscular control strategies in non-avian theropods, and how these aspects evolved on the line to birds. The results support the hypothesis that an upright femoral posture is correlated with bending-dominant bone loading and abduction-based muscular support of the hip, whereas a crouched femoral posture is correlated with torsion-dominant bone loading and long-axis rotation-based muscular support. Moreover, the results of this study 
also support the inference that hindlimb posture, bone loading mechanics and muscular support strategies evolved in a gradual fashion along the line to extant birds. 


\section{Cancellous bone and theropod dinosaur locomotion.}

2 Part III - Inferring posture and locomotor

3 biomechanics in extinct theropods, and its evolution

4 on the line to birds

5

6

P.J. Bishop ${ }^{1,2,3, *}$, S.A. Hocknull ${ }^{1,2,4}$, C.J. Clemente ${ }^{5,6}$, J.R. Hutchinson ${ }^{7}$, A.A. Farke ${ }^{8}$, R.S. Barrett $^{2,3}$ and D.G. Lloyd ${ }^{2,3}$.

${ }^{1}$ Geosciences Program, Queensland Museum, Brisbane, Queensland, Australia.

${ }^{2}$ School of Allied Health Sciences, Griffith University, Gold Coast, Queensland, Australia.

${ }^{3}$ Gold Coast Orthopaedic Research, Engineering and Education Alliance, Menzies Health Institute Queensland.

${ }^{4}$ School of Biosciences, University of Melbourne, Melbourne, Victoria, Australia.

${ }^{5}$ School of Science and Engineering, University of the Sunshine Coast, Maroochydore, Queensland, Australia.

${ }^{6}$ School of Biological Sciences, The University of Queensland, Brisbane, Queensland, Australia.

${ }^{7}$ Structure and Motion Laboratory, Department of Comparative Biomedical Sciences, Royal Veterinary College, Hatfield, Hertfordshire, UK.

${ }^{8}$ Raymond M. Alf Museum of Paleontology at The Webb Schools, Claremont, California, USA.

*corresponding author at current address: Structure and Motion Laboratory, Department of Comparative Biomedical Sciences, Royal Veterinary College, Hatfield, Hertfordshire, UK; pbishop@,rvc.ac.uk. 
29 III.1 Abstract

30

31

32

This paper is the last of a three-part series that investigates the architecture of cancellous bone in the main hindlimb bones of theropod dinosaurs, and uses cancellous bone architectural patterns to infer locomotor biomechanics in extinct non-avian species. Cancellous bone is highly sensitive to its prevailing mechanical environment, and may therefore help further understanding of locomotor biomechanics in extinct tetrapod vertebrates such as dinosaurs. Here in Part III, the biomechanical modelling approach derived previously was applied to two species of extinct, nonavian theropods, Daspletosaurus torosus and Troodon formosus. Observed cancellous bone architectural patterns were linked with quasi-static, three-dimensional musculoskeletal and finite element models of the hindlimb of both species, and used to to derive characteristic postures that best aligned continuum-level principal stresses with cancellous bone fabric. The posture identified for Daspletosaurus was largely upright, with a subvertical femoral orientation, whilst that identified for Troodon was more crouched, but not to the degree observed in extant birds. In addition to providing new insight on posture and limb articulation, this study also tested previous hypotheses of limb bone loading mechanics and muscular control strategies in non-avian theropods, and how these aspects evolved on the line to birds. The results support the hypothesis that an upright femoral posture is correlated with bending-dominant bone loading and abductionbased muscular support of the hip, whereas a crouched femoral posture is correlated with torsiondominant bone loading and long-axis rotation-based muscular support. Moreover, the results of this study also support the inference that hindlimb posture, bone loading mechanics and muscular support strategies evolved in a gradual fashion along the line to extant birds. 


\section{III.2 Introduction}

62

The non-avian theropod dinosaurs include some of the most recognisable of extinct animals, and with the carnivorous lifestyle and large body size of many species, they have received much attention concerning various aspects of their palaeobiology (e.g., Alexander 1989; Bakker 1986; Brusatte et al. 2010; Horner \& Lessem 1993; Molnar \& Farlow 1990). Locomotion in particular is a well-studied (and sometimes controversial) topic, not only because of the interest in how a giant, bipedal predator may have functioned, but also because it was likely intimately tied to the evolution of the living decendants of non-avian dinosaurs, the volant birds (Allen et al. 2013; Gatesy 1990; Gatesy 1995; Gatesy 2002; Gatesy \& Middleton 1997; Hutchinson \& Allen 2009). A variety of different approaches and lines of evidence have been previouly used to address questions of locomotor biomechanics in non-avian theropods and its evolution on the line to birds, including fossil footprints (Farlow et al. 2012; Gatesy et al. 1999; Thulborn 1990), external bone shapes and proportions (Carrano 1998; Carrano 2000; Gatesy \& Middleton 1997; Paul 1998), bone scaling (Carrano 2001; Christiansen 1999; Gatesy 1991), midshaft cortical geometry (Alexander 1989; Christiansen 1998; Farlow et al. 1995) and muscle attachments and significance (Carrano \& Hutchinson 2002; Gatesy 1990; Hutchinson 2001a; Hutchinson 2001b). These have been more recently augmented with various computational biomechanical models, that have examined aspects such as speed capabilities (Gatesy et al. 2009; Hutchinson 2004; 2012; Bates et al. 2012; Hutchinson et al. 2005; Hutchinson et al. 2008) and mass properties (Allen et al. 2013; Allen et al. 2009; Bates et al. 2012; Bates et al. 2009a; Bates et al. 2009b; Henderson 1999; Henderson \& Snively 2003; Hutchinson et al. 2011; Hutchinson et al. 2007)

The collective result of this prolonged and intensive research focus has been a much refined understanding of how anatomy influenced non-avian theropod stance and gait, and how these may have evolved on the line to extant birds. For instance, most non-avian species are inferred to have used a largely upright hindlimb posture during normal locomotion, where the hips and knees were flexed only to a minor degree; however, more crownward clades (e.g., paravians) may have used a more crouched posture with greater flexion at the hip and knee (Hutchinson \& Allen 
91 2009). These postural changes are inferred to have occurred in association with changes in other

92 biomechanically important aspects, including an anterior shift in the location of the whole-body centre of mass (COM; Allen et al. 2013), the muscular mechanisms of limb support and propulsion (Gatesy 1990; Gatesy 1995; Gatesy 2002; Hutchinson \& Gatesy 2000) and bone loading mechanics (Carrano 1998). Yet, despite important advances in understanding, there is still potential for further advances to be made, from investigation of hitherto unstudied lines of evidence. One such line of evidence is the architecture of cancellous bone, which is well known from studies of extant animals to be highly sensitive and well adapted to its prevailing mechanical environment (cf. Part I of this series; Bishop et al. in review-b). Study of cancellous bone architectural patterns in non-avian theropods may therefore provide new and unique insight into various aspects of non-avian theropod locomotor biomechanics.

In Part I of this series, stark differences in hindlimb cancellous bone architecture were found between humans and birds, the only obligate bipeds alive today. Many of these differences can be associated with differences in the manner of striding, parasagittal, bipedal locomotion employed by the two groups. In particular, the differences in cancellous bone architecture reflect differences in their upright versus crouched postures and subsequent whole-bone loading mechanics, that is, the prominence of bending and torsion. The different postures employed by humans and birds are also associated with the mechanism of muscular control required to achieve limb support during locomotion. In humans, mediolateral collapse of the stance phase limb is counteracted by hip abduction, conferred predominantly by the gluteal muscles located dorsal to the hip (Pauwels 1980; Wall-Scheffler et al. 2010). However, in birds, anatomical, kinematic and electromyographic evidence suggests that stance limb collapse is counteracted predominantly by medial (internal) long-axis rotation of the subhorizontally oriented femur, conferred by the iliotrochantericus muscles located anterior to the hip (Gatesy 1999b; Hutchinson \& Gatesy 2000). But what of extinct obligate bipeds, such as non-avian theropod dinosaurs?

In more stemward species of non-avian theropod, the architecture of cancellous bone in the main hindlimb bones is similar to that of humans, in terms of both principal fabric directions in the hip and knee and whole-bone architectural patterns. For instance, there exists a double-arcuate pattern in the proximal femur, roughly parallel to the coronal plane; this was not observed in 
122 more crownward non-avian species or extant birds (Part I; Bishop et al. in review-b). In species

123 more closely related to extant birds, cancellous bone architecture tends to be more similar to that 124 observed in birds. For instance, in the diaphysis-ward parts of the femoral metaphysis, primary 125 fabric vectors are disorganized and often oblique to the long-axis of the bone; and in Paravians 126 and extant birds at least, the distal tibiotarsus shows a distinctive and strongly anisotropic pattern 127 of sagitally aligned, often plate-like trabeculae (Part I; Bishop et al. in review-b). Given that 128 cancellous bone architectures in extant obligate bipeds appear to be linked to their different 129 locomotor biomechanics, these observations raise the following questions regarding non-avian 130 theropods:

131 1. Did the different species of non-avian theropods employ different limb postures?

132 2. Did the bones of the different species of non-avian theropods experience different loading 133 regimes?

134 3. Did the different species of non-avian theropods employ different strategies of muscular 135 support in counteracting stance limb collapse?

136 4. If the different species of non-avian theropods did employ different suites of hindlimb 137 locomotor biomechanics, how did these evolve on the line to extant birds?

138 Previously, the integration of anatomical, kinematic, bone strain and electromyographic data in 139 extant species led Carrano (1998) and Hutchinson \& Gatesy (2000) to hypothesize that the 140 aforementioned aspects of bipedal locomotor biomechanics were intimately tied throughout 141 theropod evolution. The incremental change of external osteological features throughout theropod 142 evolution was also taken to indicate that the transformation in these particular biomechanical 143 aspects was a gradual occurrence (Hutchinson 2001a; Hutchinson 2001b; Hutchinson \& Gatesy 144 2000). More broadly however, the exact history of theropod locomotor evolution, in terms of 145 whether it was long and gradual, or more punctuated at certain instances, remains to be fully 146 discerned (Allen et al. 2013; Hutchinson \& Allen 2009).

A new approach that can quantitatively address the aforementioned questions was outlined in Part II of this series (Bishop et al. in review-a). In this 'reverse trajectorial approach', the observed three-dimensional (3-D) architecture of cancellous bone in the main bones of the hindlimb is 151 coupled with musculoskeletal and finite element models of the hindlimb. Under a quasi-static 152 situation, these models are used to derive a single 'characteristic posture', one in which 
153 continuum-level principal stresses best align with cancellous bone fabric. This characteristic

154 posture is a time- and load-averaged posture across all loading regimes, and it is important to 155 recognize that it may or may not be an actual posture used at a particular instance in a particular 156 behaviour.

157

158

159

160

161

162

163

164

165

166

167

168

169

170

171

172

173

174

175

176

177

178

179

180

181

182

In Part II it was shown that when applied to an extant theropod (chicken, Gallus gallus), the new approach was able to retrieve a posture that was quite comparable to that used by birds at around the midstance of normal terrestrial locomotion. It could also provide a reasonable assessment of bone loading in the proximal limb (i.e., femur, proximal tibia and proximal fibula) and muscle control strategies for limb stabilization, although it had markedly lower accuracy in terms of bone loading in the distal limb (tibial shaft and below) and muscle control strategies for limb propulsion. Additionally, it was shown that the results of this approach were largely insensitive to actual muscle size (manifest as force-generating capacity), a key unknown for extinct species. When applied to extinct, non-avian theropods, the approach may therefore be used to investigate posture, bone loading mechanics and muscle recruitment patterns in these species as well. Thus, in this approach the architecture of cancellous bone constitutes an independent data set against which one or more biomechanical hypotheses may be tested.

The present study aimed to quantitatively test the hypotheses of Carrano (1998) and Hutchinson \& Gatesy (2000) concerning the evolution of theropod locomotor mechanics. To do this, it applied the reverse trajectorial approach to two species of non-avian theropod, the phylogenetically basal coelurosaur Daspletosaurus torosus and the phylogenetically derived paravian Troodon formosus, to derive a single characteristic hindlimb posture that best reflects these species' architectural patterns of cancellous bone. These species show markedly different cancellous bone architectures, with that of the former more similar to that of humans and that of the latter bearing stronger resemblance to that of birds (Part I). Understanding limb posture in these and other non-avian theropod species is in and of itself important, but it is also important for understanding other aspects of locomotion. For instance, posture can influence maximum speed capability in bipeds (Gatesy et al. 2009; Hutchinson 2004; Hutchinson \& Allen 2009). In concert with the results already derived from for an extant bird, the chicken (Part II), the results 
183 of this study will also facilitate an examination of how locomotor biomechanics has evolved in

184 theropods on the line to extant birds.

185

186

187

188

189

190

191

192

193

194

195

196

197

198

199

200

201

202

203

204

205

206

207

208

209

210

211

212

213

\section{III.3 Materials and Methods}

The methodology employed in the present study followed that outlined previously in Part II (Bishop et al. in review-a). Essentially, musculoskeletal models of the hindlimb in a static posture were used to provide the force and boundary conditions for finite element modelling of the individual limb bones, from which principal stress trajectories were determined and compared to cancellous bone architectural patterns; the degree of correspondence between stress trajectories and cancellous bone fabric was then used to inform a new test posture. This was repeated until no further improvement in overall correspondence across the femur, tibiotarsus and fibula was able to be gained; at this point the 'solution posture' was achieved. Only those differences from the methodology of Part II, associated with the modelling of the two different species, will be described in the present study. Also, as with the previous study, all assumptions and model parameters were kept in their 'best guess' manifestation throughout the analyses; thus, differences in model results directly reflected differences in limb postures in the extinct species.

All scripts, models and data used are held in the Geosciences Collection of the Queensland Museum, and are available upon request to the Collections Manager. Additionally, a complete copy of the raw data derived from the fossil specimens is accessioned with the respective museums in which the specimens are housed.

\section{III.3.1 Skeletal geometry acquisition}

The models developed in this study were derived through a combination of X-ray computed tomographic (CT) scanning and photogrammetry of multiple fossil specimens; see Table 1 for the 
214 specimens (and institutional abbreviations) and imaging parameters used. The CT scans for each

215 specimen were segmented using the software Mimics 17.0 (Materialize NV, Belgium), via a

216 combination of manual and automatic techniques, to produce initial surface meshes of each bone.

217 For photogrammetry, digital photographs were taken with a Lumix DMC-TZ40 (Panasonic,

218 Japan) and rendered to produce 3-D meshes using the software Agisoft Photoscan 1.0.4 (Agisoft

219 LLC, Russia), RealityCapture 1.0 (Capturing Reality s.r.o., Slovakia), Meshlab 1.3.3

220 (http://meshlab.sourceforge.net/) and CloudCompare 2.5.4 (http://www.cloudcompare.org/).

221

222

223

224

225

226

227

228

229

230

231

232

233

234

235

236

237

238

239

240

241

242

243

244

To maximize rigour, the models for each species were based primarily on single focal individuals that were relatively complete and well-preserved, and for which information on cancellous bone architecture was previously reported (Part I). These were TMP 2001.036.0001 for Daspletosaurus and MOR 748 for Troodon. At the time the research was undertaken, the specimens used to produce the model for Troodon were believed to represent a single species, Troodon formosus. However, recent research has indicated otherwise, and furthermore has cast doubt on the validity of the name Troodon formosus itself (van der Reest \& Currie 2017); the majority of specimens used in this study therefore belong to an unnamed taxon. Nonetheless, the model constructed here is still considered to be an accurate reflection of the anatomy of a large, phylogenetically derived, North American troodontid. Moreover, for the sake of simplicity in the present study, the animal being modelled will herein be referred to as 'Troodon'.

Some bones, or parts thereof, were missing from these focal specimens, and in these cases their geometry was modelled using other specimens of the same or closely related species (Table 1). This was achieved by scaling the geometries of these other specimens appropriately to fit the focal specimens' bones, accomplished using a combination of Mimics and the computer-aided design software Rhinoceros 4.0 (McNeel, USA). Wholesale reconstruction was required for the much of the pubis in Daspletosaurus and much of the ilium in 'Troodon'. In Daspletosaurus, the general shape of the pubis was evident from the focal specimen, but much of the boot, pubic apron and ischiadic head were reconstructed based on comparison to other specimens that were imaged (Table 1), personal observation of other specimens in the TMP and MOR collections, and also the tyrannosaurid literature (e.g., Brochu 2003; Osborn 1917). In 'Troodon', the acetabulum, antitrochanter and pubic and ischiadic peduncles were present in the focal specimen, but the 
245 anterior and posterior iliac blades were reconstructed based on comparison to other troodontids

246 described in the literature (e.g., Gao et al. 2012; Tsuihiji et al. 2014; Xu et al. 2002). The

247 assembly of the individual elements of the pelvis was based on the geometry of individual bones,

248 but also on specimens of other tyrannosaurids or paravians where the pelvic elements were

249 preserved in situ and intact with the sacrum (e.g., Brochu 2003; Gao et al. 2012; Lambe 1917;

250 Norell \& Makovicky 1997; Osborn 1917; Tsuihiji et al. 2014; Xu et al. 2002), as well as personal

251 observation of other specimens in the TMP and MOR collections and displays. For completeness,

252 the vertebral column was represented by a single cylinder fixed with respect to the pelvis. In

253 addition to the pelvis, the distalmost fibular shaft was also reconstructed for 'Troodon'; it was

254 essentially a continuation of the preserved part of the shaft, tapering towards the end, and gently

255 curving laterally as it approaches the distal tibia (cf. Norell \& Makovicky 1999; Ostrom 1969).

256

257 Some of the individual bones used in the above procedure had undergone a variable amount of

258 taphonomic distortion. However, in all cases this appeared to be brittle deformation only, in the

259 form of fracturing and rigid displacement of the fragments relative to one another. In these

260 instances, the bones were retro-deformed in Rhinoceros, under the assumption of brittle

261 deformation (Lautenschlager 2016). This rigid retro-deformation restored the fossil geometry

262 closer to the original geometry by realigning fragments along apposing fracture surfaces, and also

263 taking into consideration the geometry of the bones in other specimens and other species,

264 including comparison to the literature (e.g., Brochu 2003; Tsuihiji et al. 2014). The retro-

265 deformed geometries were then 'smoothed over' in Mimics and 3-Matic 9.0 (Materialize NV,

266 Belgium). Additionally, cracks or abraded edges were filled in and reconstructed in Mimics; only

267 the minimal amount of filling in required was undertaken.

268

269 Once an initial surface mesh had been produced for the complete geometry of each bone for both

270 species, these were smoothed in 3-matic and then refined to produce a more isoparametric mesh

271 in ReMESH 2.1 (Attene \& Falcidieno 2006; http://remesh.sourceforge.net/). Although the tibia,

272 astragalus and calcaneum typically remain as separate ossifications in tyrannosaurids, and the

273 tibia remains separate from the astragalus and calcaneum in troodontids, the meshes of the three

274 bones were fused together in this study to create a single tibiotarsus geometry. This was 
275

276

277

278

279

280

281

282

283

284

285

286

287

288

289

290

291

292

293

294

295

296

297

298

299

300

301

302

303

304

305

undertaken for the sake of simplifying the models, as well as maintaining a greater degree of consistency with the previously developed chicken model of Part II.

\section{III.3.2 Musculoskeletal modelling}

Musculoskeletal models of the right hindlimb of Daspletosaurus and 'Troodon' were constructed in NMSBuilder (Martelli et al. 2011; Valente et al. 2014) for use in OpenSim 3.0.1 (Delp et al. 2007), and are shown in Figs 1 and 2. Both comprised 12 degrees of freedom, as in the chicken model of Part II, and 38 musculotendon actuators.

\section{$\underline{\text { III.3.2.1 Definition of joints }}$}

Joint locations and orientations were defined in a similar fashion to the chicken model. However, the location of the hip joint was left open-ended, so as to investigate the effects of different hip articulations (see Section III.3.5 below). Initially, the centre of the joint in the femur was determined by fitting a sphere to the femoral head in 3-matic, and the centre of the joint in the acetabulum was determined by positioning the centre of femoral head sphere in the centre of the acetabulum (in both lateral and anterior views). Hence, in this initial configuration, the articulation of the femur with the acetabulum was consistent with the configuration used for the chicken model. It was also consistent with the inference drawn in Part I from observations of cancellous bone architecture (Section I.5.2.3), that the articulation was possibly centred about the apex of the femoral head. The articulation of the tibia and fibula was guided by the relative positions of the fibular crest on the tibiotarsus and the flared anteromedial process of the proximal fibula, as well as the facet formed distally by the tibia, astragalus and calcaneum for reception of the fibula. As with the chicken model, the pes was modelled as a rectangular prism, with a width set to the mediolateral width of the distal tarsometatarsus and a length set to the total 
306 length of digit III; the total length of digit III for the 'Troodon' model was based on the data of

307 Russell (1969) for Latenivenatrix mcmasterae, scaled to the individual modelled in the current 308 study.

309

310

311

III.3.2.2 Definition of muscle and ligament anatomy

312

313 The hindlimb myology of Daspletosaurus and 'Troodon' was reconstructed through analysis of 314 the muscle and ligament scarring patterns observed on the fossil bones, framed in the context of 315 the myology and scarring patterns of extant archosaurs (Bates \& Schachner 2012; Bates et al. 316 2012; Carrano \& Hutchinson 2002; Hutchinson 2001a; Hutchinson 2001b; Hutchinson 2002;

317 Hutchinson et al. 2005; Hutchinson et al. 2008). The 33 muscles and four ligaments

318 reconstructed, along with their origins and insertions, are listed in Table 2. As in the chicken

319 model, the collateral ligaments of the knee and ankle were represented by four musculotendon 320 actuators in both the Daspletosaurus and 'Troodon' models. Each muscle was represented by a

321 single musculotendon actuator in the models, with one exception; the iliotibialis 2 (IT2) was

322 represented by two actuators on account of its probable expansive origin on the dorsal ilium

323 (Bates et al. 2012; Hutchinson et al. 2005; Hutchinson et al. 2008). The 3-D courses of the

324 actuators were constrained to follow paths that are comparable to those reported for homologous

325 muscles in extant archosaurs, and also as reconstructed for other non-avian theropod species

326 (Bates \& Schachner 2012; Bates et al. 2012; Hutchinson et al. 2005; Hutchinson et al. 2008).

327

328

329

In reconstructing the muscular and ligamentous components of the models, a number of 330 simplifying assumptions were made. Two muscles, the ambiens (AMB) and fibularis longus (FL) may possibly have sent off secondary tendons to attach more distally in the limb, as can occur in extant archosaurs (Carrano \& Hutchinson 2002; Hutchinson 2002). However, these secondary attachments were assumed to be of little importance for bone loading mechanics as far as the present study is concerned, and so were not modelled. A distal accessory tendon was considered to be absent from the caudofemoralis longus (CFL), as the fourth trochanter of both species lacks a distally directed process or is of small size (Carrano \& Hutchinson 2002; Hutchinson 2001a). It is also possible that there may have been other flexor muscles of digits II-IV in both 
337 Daspletosaurus and 'Troodon', in addition to the flexores digitorum longus (FDL) et brevis

338 (FDB), but currently it is too speculative to infer these (Carrano \& Hutchinson 2002; Hutchinson

339 2002). It was assumed in the present study that if any such digital flexor muscles were present in

340 either species, they would have had a similar disposition to the FDL, and so their action could be

341 represented by the FDL actuator.

342

343

344

345

346

347

III.3.2.3 Definition of segment mass properties

348

349

350

To estimate the mass properties of each limb segment in the Daspletosaurus musculoskeletal model, the segment soft tissue models of Allen et al. (2013) for Tyrannosaurus were modified to 351 fit the pelvic and limb elements of Daspletosaurus, by scaling each soft tissue segment in the $x, y$ and $z$ directions to fit the relevant bone or bones (and in the case of the thigh segment, to also fit the pelvis). This was accomplished in Rhinoceros. Likewise, the segment soft tissue models of Allen et al. (2013) for Velociraptor were modified appropriately to fit the pelvic and limb elements of 'Troodon' in the estimation of mass properties in its model. The application of the soft tissue models developed for other species to the species studied here is justified, due to close phylogenetic relationship and much similarity in the underlying skeletal structure between the species involved. Assuming a bulk density of $1000 \mathrm{~kg} / \mathrm{m}^{3}$ for all body segments, the total mass of the right hindlimb in the Daspletosaurus model was calculated to be $342.7 \mathrm{~kg}$, and that in the 'Troodon' model was $5.65 \mathrm{~kg}$.

To completely define the musculoskeletal model, this also required the calculation of mass properties for the remainder of the body, that is, the pelvis segment of the models. Based on femoral mid-shaft circumferences, equation 7 of Campione et al. (2014) was used to estimate the total body mass for the two models. This resulted in a mass of $2757 \mathrm{~kg}$ for the Daspletosaurus model and $48.5 \mathrm{~kg}$ for the 'Troodon' model, and hence the mass of the pelvis segment in the two models (including the mass of the left hindlimb) was $2414.3 \mathrm{~kg}$ and $42.85 \mathrm{~kg}$, respectively. By 
368

369

370

371

372

373

374

375

376

377

378

379

380

381

382

383

384

385

386

387

388

389

390

391

392

393

394

395

396

397

398

unintended coincidence, in both models the mass of the right hindlimb constituted approximately $12 \%$ of total body weight, which therefore increased consistency between two models. For comparison, the mass of the hindlimb in the chicken model of Part II constituted approximately $10 \%$ of total body weight. Given the data reported by Allen et al. (2013), the combined COM of the whole body, minus the right leg, in their 'average' model of Tyrannosaurus was $0.544 \mathrm{~m}$ anterior to the hip joint. The femur length of the specimen upon which their model was based is $1.265 \mathrm{~m}$, as reported by Hutchinson et al. (2011). Scaling isometrically to the Daspletosaurus model, which has a femur length of $0.984 \mathrm{~m}$, the COM of the pelvis segment was set at $0.423 \mathrm{~m}$ anterior to the hip. Similarly, the combined COM of the whole body, minus the right leg, in the 'average' Velociraptor model of Allen et al. (2013) was $0.090 \mathrm{~m}$ anterior to the hip joint, and the femur length upon which their model was based is $0.163 \mathrm{~m}$. Thus, scaling isometrically to the 'Troodon' model, which has a femur length of $0.304 \mathrm{~m}$, the COM of the pelvis segment was set at $0.168 \mathrm{~m}$ anterior to the hip. The dorsoventral position of the COM of the pelvis segment was assumed to be level with the hip. As noted in Part II, the dorsoventral position of the pelvis segment COM will not influence the results so long as the pelvis segment's orientation was fixed in all simulations, and all simulations were quasi-static in nature.

\section{III.3.2.4 Muscle activity}

Not all of the 34 musculotendon actuators representing muscles were set to be active during the musculoskeletal simulations, in both Daspletosaurus and 'Troodon' (Table 3). The inactive muscles were set using the same criteria employed for the chicken model, and through comparison to published electromyography data for homologous hindlimb muscles in extant archosaurs (Gatesy 1990; Gatesy 1994; Gatesy 1997; Gatesy 1999b; Jacobson \& Hollyday 1982; Marsh et al. 2004; Reilly \& Blob 2003; Roberts et al. 1998). One exception to this was the iliofemoralis externus (IFE), which in both birds and crocodilians is mostly active during the swing phase of locomotion. However, in the evolutionary scenario proposed by Hutchinson \& Gatesy (2000), abductor muscles such as the IFE are expected to have been crucial to maintaining stance limb stability, if the femur was habitually held in the subvertical orientation hypothesized for most, if not all, non-avian theropods (Hutchinson \& Allen 2009). Moreover, the hypothesis of 
399 Hutchinson \& Gatesy (2000) explains the stance phase inactivity of the IFE (or its homologues)

400 in birds and crocodilians as a result of other hip muscles conferring stance limb support, namely, 401 medial long-axis rotators in birds (iliotrochanterici) and adductors in crocodilians (adductores

402 femoris 1 et 2). Thus, to test the hypothesis of Hutchinson \& Gatesy (2000), among others, the 403 IFE was set as being active in both the Daspletosaurus and 'Troodon' simulations. All active 404 musculotendon actuators were assigned the same maximum force capacity, equal to two times 405 body weight, that is, each muscle was capable of exerting up to $54073.9 \mathrm{~N}$ for Daspletosaurus 406 and $951.2 \mathrm{~N}$ for 'Troodon'.

407

As in the chicken simulations of Part II, a reserve actuator was applied to the metatarsophalangeal joint in the musculoskeletal simulations. The maximum output of this actuator in the Daspletosaurus and 'Troodon' simulations was scaled from that set for the chicken $(1,000 \mathrm{Nm})$, in proportion to the total body mass of each model: 1,767,308 Nm for Daspletosaurus and 31,090 Nm for 'Troodon'. This corresponds to a minimum of 27 times the product of body weight and total hindlimb length (sum of interarticular lengths of femur, tibiotarsus and tarsometatarsus). By providing ample control of the metatarsophalangeal joint, this helped reduce excessively high recruitment of the FDL and FDB.

\section{$\underline{\text { III.3.2.5 Initial posture }}$}

419

A general mid-stance posture was used as an initial starting point, which was modified in subsequent modelling attempts, as per the process outlined in Part II of this study. This initial posture was based on general interpretations of tyrannosaurid and troodontid appearance in the literature (technical and popular). Additionally, the hip extension angle was initially set so that the knee joint was near the line of the vertical ground reaction force in the $x-z$ (sagittal) plane,

425 following previous interpretations of theropod hindlimb biomechanics (Gatesy et al. 2009; Hutchinson \& Gatesy 2006). 
431 Finite element simulations of the Daspletosaurus and 'Troodon' models were developed and

432

433

434

435

436

437

438

439

440

441

442

443

444

445

446

447

448

449

450

451

452

453

454

455

456

457

458

459

460 solved in largely the same manner as the previously described chicken simulations of Part II, using ANSYS 17.0 (Ansys, Inc., USA). Two minor differences were that (i) a graduated and finer mesh was used around the cleft of the lesser trochanter of the Daspletosaurus femur, to reduce stress artifacts, and (ii) connection between the tibiotarsus and fibula entities was modelled both proximally and distally. The latter difference reflects that fact that both tyrannosaurs and troodontids possessed a distinct furrow in the distal tibiotarsus for reception of the distal fibula, whereas in birds the distal fibula is greatly reduced. In the Daspletosaurus model, the total number of elements used across the various postures tested ranged from 961,023 to 975,544 in the femur simulation and from 985,071 to $1,005,550$ in the tibiotarsus + fibula simulation. In the 'Troodon' model, the total number of elements used across the various postures tested ranged from 668,033 to 684,547 in the femur simulation and from 583,228 to 598,556 in the tibiotarsus + fibula simulation.

\section{III.3.4 Results analysis}

In Part II, stress trajectories for the chicken model were compared to the observed cancellous bone architecture in birds as a whole (reported in Part I), for reasons explained there. Here, stress trajectories for the Daspletosaurus model were compared to observed cancellous bone architecture in Allosaurus and tyrannosaurid fossils, and stress trajectories for the 'Troodon' model were compared to observed architectural patterns in troodontid fossils. Qualitative comparisons of stress trajectories to fabric directions were made across all three bones: femur, tibiotarsus and fibula. Supplementing these qualitative assessments, quantitative comparison of stresses and architecture was undertaken for the femoral head and medial femoral condyle, followed the procedure outlined for the chicken model in Part II. The direction of minimum principal stress $\left(\boldsymbol{\sigma}_{3}\right)$ was determined as the mean direction of vectors within anatomically scaled and positioned spheres placed within each region of the bone, with the mean principal fabric direction in both regions taken as previously reported in Part I (figs 22, 29). 
461

462

463

464

465

466

467

468

469

470

471

472

473

474

475

476

477

478

479

480

481

482

483

484

485

486

487

488

489

490

491

\section{III.3.5 Varying hip articulation}

Following the identification of a 'solution posture' for the Daspletosaurus model, a brief exploratory exercise was undertaken to address the ambiguity surrounding the articulation of non-avian theropod hips. Unlike birds, many non-avian theropods typically possessed a large incongruence in size between the femoral head and the acetabulum; for example, in the Daspletosaurus focal specimen studied, the diameter of the femoral head is about two-thirds that of the acetabulum (Fig. 3). This has consequently created uncertainty in exactly how the femur articulated with the acetabulum in these extinct species (see also Tsai \& Holliday 2015; Tsai et al. 2018). It has been previously suggested that the main area of articulation on the femur occurred on the roughly cylindrical part of the femoral head, lateral to the apex of the head (e.g., Hotton 1980; Hutchinson \& Allen 2009). However, cancellous bone architectural patterns observed in Allosaurus and tyrannosaurids (Part I) suggest that hip joint loads may have been transmitted through the femoral head mainly from the apex of the head, not from the more lateral parts.

To examine the effect of different hip articulations in the Daspletosaurus model, the extent of femur-acetabulum contact was varied to assess if any improvement in correspondence between principal stress trajectories and cancellous bone architecture was possible beyond that of the solution posture (Fig. 3). Two such variations were made. Firstly, the femur was moved $50 \mathrm{~mm}$ medially with respect to the acetabulum, so that a sizeable proportion of the cylindrical part of the femoral head was in close proximity to the acetabulum (Fig. 3D-F). The rest of the limb was also moved medially along with the femur, including the coordinate systems of distal joints and all musculotendon actuator origins, insertions and via points that were level with or distal to the hip. So as to maintain a similar mediolateral foot placement as the original solution posture, the amount of hip abduction-adduction was altered slightly. In the second variation, the femur and limb distal to it was again moved $50 \mathrm{~mm}$ medially with respect to the acetabulum, but the hip was also abducted by $14^{\circ}$, producing a net $10^{\circ}$ abduction from the neutral posture (Fig. $\left.3 \mathrm{G}-\mathrm{I}\right)$. This reflects the amount of hip abduction that has been supposed for tyrannosaurids in previous modelling studies (e.g., Hutchinson et al., 2005, 2007), on account of the inclined disposition of the femoral head relative to the long-axis of the femur. In order to bring the foot anywhere near the body midline, this abducted posture also necessitated a large $27^{\circ}$ of external long-axis 
492 rotation of the hip, a value comparable to maximal external long-axis rotation in modern birds

493 during straight-line locomotion (Kambic et al. 2015; Rubenson et al. 2007).

494

495

496

\section{III.3.6 Cross-species patterns}

497

498

499

Once solution postures were identified for both the Daspletosaurus and 'Troodon' models, a number of biomechanically relevant parameters were extracted. The same parameters were also extracted from the solution posture identified previously for the chicken model of Part II. By way

501

502

503

504

505

506

507

508

509

510

511

512

513

514

515

516

517

518

519

520

521 of comparison across the three species, these parameters would allow a quantitative assessment of the evolutionary-biomechanical hypotheses of Carrano (1998) and Hutchinson \& Gatesy (2000). Three sets of parameters were extracted:

1. Postural parameters, related to Question 1 posed in the Introduction: the location of the whole-body COM as normalized by total hindlimb length, joint angles for the hip and knee, and the 'degree of crouch', both actual and predicted from empirical data reported by Bishop et al. (2018).

2. Bone loading parameters, related to Question 2 posed in the Introduction: the orientation of principal stresses at the femoral mid-shaft, the ratio of maximum shear stress to bending stresses at the femoral mid-shaft, and the orientation of the neutral surface of bending at the femoral mid-shaft, relative to the mediolateral axis. To enable estimation of these parameters at mid-shaft, a local long-axis in the vicinity of the mid-point of the bone was determined. This was calculated by fitting a cylinder to the shaft in the immediate vicinity of the midpoint, using the in-built cylinder fitting tool in 3-matic; the long-axis of the cylinder defined the local long-axis of the bone, and the plane normal to this axis defined the plane of the mid-shaft cross-section. The orientation of principal stresses was defined as the orientation of the steepest inclined stress vector with respect to the local long-axis; this was calculated separately for both $\boldsymbol{\sigma}_{1}$ and $\boldsymbol{\sigma}_{3}$, and then the mean orientation was taken. In pure bending the orientation would be $0^{\circ}$, that is, parallel to the long-axis, and in pure torsion it would be $45^{\circ}$ (Beer et al. 2012). Additionally, mid-shaft bending stresses were calculated as

$$
\sigma_{\text {bending }}=\frac{\left|\sigma_{\max }\right|+\left|\sigma_{\min }\right|}{2},
$$


522

523

524

525

526

527

528

529

530

531

532

533

534

535

536

537

538

539

540

541

542 Given the small sample size of species examined here $(n=3)$, any assessment of the evolution of

543

544

545

546

547

548

549

550

551

where $\sigma_{\max }$ is the maximum (tensile) stress at mid-shaft and $\sigma_{\min }$ is the minimum (compressive) stress at mid-shaft. This assumes that planar strain conditions were in place (Biewener 1992), which was revealed by inspection of normal stress contours to be approximately true.

3. Muscular support parameters, related to Question 3 posed in the Introduction: the abduction moments of muscles that are predominantly suited to conferring hip abduction (i.e., iliofemoralis externus), and the long-axis rotation moments of muscles that are predominantly suited to conferring hip long-axis rotation (i.e., iliotrochantericus caudalis and puboischiofemorales internus 1 et 2 in non-avian theropods; iliotrochanterici caudalis et medialis in the chicken). To give a size-independent, dimensionless measure of how much 'effort' a muscle exerts to stabilize a joint about a given axis, these moments are normalized by the product of the model's body weight and hip height:

$$
M^{*}=\frac{a \cdot F_{\max } \cdot r_{i}}{m \cdot g \cdot h},
$$

where $a$ is the activation level of the muscle, from 0 (inactive) to 1 (maximally active), $F_{\max }$ is the maximum force capable of being produced (set at two body weights as per Part II), $r_{i}$ is the muscle's moment arm about joint axis $i, m$ is body mass, $g$ is the acceleration due to gravity $\left(9.81 \mathrm{~m} / \mathrm{s}^{2}\right)$ and $h$ is hip height. It is worth noting that this analysis carries the caveat of ignoring biarticular muscles (e.g., iliotibiales) and co-contraction between agonistic and antagonistic muscles.

biomechanically relevant parameters is necessary a coarse one. Since the hindlimb anatomy of Daspletosaurus is close to that inferred for the ancestral state of Coelurosauria, its results may taken to be reasonably representative of the most recent common ancestor of it and 'Troodon'; likewise, since the anatomy of the 'Troodon' model is close to that inferred for the ancestral state of Paraves, its results may taken to be reasonably representative of the most recent common ancestor of it and the chicken. That is, it is here assumed that - in the context of locomotor biomechanics - little important evolution occurred between the ancestral coelurosaur and Daspletosaurus, and likewise little important evolution occurred between the ancestral paravian and 'Troodon'. By mapping results towards the most recent common ancestor of successive 
552 clades, the differences observed between Daspletosaurus, 'Troodon' and the chicken are hence

553 taken to be a surrogate for the actual sequence (if not pattern) of evolution along the avian stem

554 lineage. This does not, however, escape the caveat of allometric effects on dimensional aspects of

555 hindlimb anatomy; the issue of size effects in theropod locomotor evolution will be returned to in

556 the Discussion.

557

558

\section{III.4 Results}

560

561

A total of five different postures for Daspletosaurus, and six postures for 'Troodon', were tested

562

before no further correspondence between principal stress trajectories and cancellous bone

563 architectural patterns was able to be achieved (Figs 4, 5). In the Daspletosaurus model, going from the worst to best postures tested, the angular deviation between the minimum compressive stress $\left(\boldsymbol{\sigma}_{3}\right)$ and the mean direction of the primary fabric orientation $\left(\mathbf{u}_{1}\right)$ in the femoral head decreased from $15.6^{\circ}$ to $7.3^{\circ}$, a $53 \%$ reduction; likewise, the angular deviation between $\boldsymbol{\sigma}_{3}$ and $\mathbf{u}_{1}$ in the medial femoral condyle decreased from $11.7^{\circ}$ to $2.8^{\circ}$, a $76 \%$ reduction. In the 'Troodon' model, going from the worst to best postures tested, the angular deviation between $\boldsymbol{\sigma}_{3}$ and $\mathbf{u}_{1}$ in the femoral head decreased from $23.8^{\circ}$ to $3.9^{\circ}$, an $84 \%$ reduction; likewise, the angular deviation between $\boldsymbol{\sigma}_{3}$ and $\mathbf{u}_{1}$ in the medial femoral condyle decreased from $28.3^{\circ}$ to $24.2^{\circ}$, a $14 \%$ reduction. The final solution posture for Daspletosaurus is illustrated in the centre of Fig. 4, and the solution posture for 'Troodon' is illustrated in the centre of Fig. 5. As with the results for the chicken model (Part II), only minimal correspondence between principal stress trajectories and cancellous bone architecture was able to be achieved in the distal tibiotarsus of either species. Little correspondence was also able to be achieved in the fibular crest of the Daspletosaurus model's tibia. Thus, the remainder of this section will focus on the more proximal parts of the hindlimb.

\section{III.4.1 Daspletosaurus results}

580

581 In the solution posture, the principal stress trajectories in the femur showed a high degree of 582 correspondence with the observed cancellous bone architecture throughout the bone (Figs 6, 7). 
583 Strong correspondence between $\boldsymbol{\sigma}_{3}$ (compressive) and cancellous bone architecture occurred in 584 the femoral head and both medial and lateral femoral condyles. This correspondence included 585 that between the mean direction of $\boldsymbol{\sigma}_{3}$ and $\mathbf{u}_{1}$ in the femoral head (Fig. 6G) and medial femoral 586 condyle (Fig. 7I). Correspondence between the maximum principal stress $\left(\boldsymbol{\sigma}_{1}\right.$, tensile) and 587 cancellous bone architecture occurred in the distal half of the fourth trochanter. Additionally, 588 three instances of a double-arcuate pattern occurred, formed by $\sigma_{1}$ and $\sigma_{3}$, largely in the coronal 589 plane. These correlate to three similar such patterns observed in the cancellous bone architecture 590 of tyrannosaurids: in the femoral head and proximal metaphysis, in the lesser trochanter, and in 591 the anterior and posterior parts of the distal femur proximal to the condyles. The double-arcuate 592 patterns of $\sigma_{1}$ and $\sigma_{3}$ sometimes also occurred in the results for other postures tested, but they 593 were often less developed compared to the solution posture.

594

Strong correspondence between principal stress trajectories and cancellous bone architecture also 596 occurred in the proximal tibia and fibula (Fig. 8). The trajectory of $\boldsymbol{\sigma}_{3}$ corresponded closely with the observed architectural patterns of both the medial and lateral condyles, including a more lateral inclination in the lateral condyle. In the cnemial crest of the tibia, the trajectory of $\boldsymbol{\sigma}_{1}$ largely paralleled the margins of the crest, as observed for cancellous bone fabric. Good correspondence between $\boldsymbol{\sigma}_{3}$ and cancellous bone architectural patterns was also observed in the fibular head, particularly for in the medial aspect of the bone (Fig. 8K,L).

\section{III.4.2 'Troodon' results}

603

604

As with the Daspletosaurus model, in the solution posture identified for 'Troodon', the principal 605 stress trajectories in the femur generally showed strong correspondence to the observed cancellous bone architecture (Figs 9, 10). Correspondence with $\boldsymbol{\sigma}_{3}$ occurred in the femoral head, under the greater trochanter and in both medial and lateral condyles; correspondence with $\boldsymbol{\sigma}_{1}$ occurred in the lesser trochanter. The mean direction of $\boldsymbol{\sigma}_{3}$ in the femoral head showed strong correspondence to the mean direction of $\mathbf{u}_{1}$ (Fig. 9E). In the medial femoral condyle, the directions of $\boldsymbol{\sigma}_{3}$ and $\mathbf{u}_{1}$ are qualitatively similar, but $\boldsymbol{\sigma}_{3}$ was notably more posteriorly inclined (by about $20^{\circ}$ ) than the mean direction of $\mathbf{u}_{1}$ (Fig. 10E), as occurred in the chicken model of Part II.

612 Unlike the results for the Daspletosaurus model, no double-arcuate pattern of $\boldsymbol{\sigma}_{1}$ and $\boldsymbol{\sigma}_{3}$ was 
613 present in 'Troodon'; instead, their trajectories tended to spiral about the bone's long axis, much

614 like the stress results for the chicken model.

615

616 Good correspondence between principal stress trajectories and cancellous bone architecture also 617 occurred in the proximal tibia and fibula (Fig. 11). In the medial and lateral condyles, $\boldsymbol{\sigma}_{3}$ 618 corresponded closely with observed architectural patterns, possessing a gentle posterior 619 inclination, with a slight lateral inclination under the lateral condyle. In the cnemial crest, the 620 trajectory of $\sigma_{1}$ largely paralleled the margins of the distal part of the crest. In the fibular head, 621 the principal stress trajectories showed good overall correspondence to the observed architectural 622 patterns (Fig. 11K-M). Greater correspondence occurred laterally with $\boldsymbol{\sigma}_{1}$, but some 623 correspondence was also present in the medial side with $\boldsymbol{\sigma}_{3}$.

624

625

626

627

628

629

630

631

632

633

634

635

636

637

638

639

640

641

642

643

\section{III.4.3 Hip articulation results}

In both variations in hip articulation tested for the Daspletosaurus model, the resulting principal stress trajectories of the proximal femur showed poorer correspondence with observed cancellous bone architecture than that achieved with the initial solution posture (Fig. 12). In particular, $\boldsymbol{\sigma}_{3}$, was broadly directed towards the more cylindrical part of the femoral head, lateral to the apex, rather than towards the apex itself. Additionally, the anterior inclination of $\sigma_{3}$ in the femoral head was greater in both variations than that in the originally identified solution posture, and was markedly greater than the anterior inclination of the mean direction of $\mathbf{u}_{1}$.

\section{III.4.4 Cross-species comparisons of biomechanical parameters}

In terms of posture, hip extension, hip adduction-abduction, hip long-axis rotation and knee flexion angles all changed in a gradual fashion progressing from Daspletosaurus to 'Troodon' to the chicken (Fig. 13). The same pattern also occurred for the anterior location of the whole-body $\mathrm{COM}$ and the degree of crouch. Furthermore, the degree of crouch of the solution postures matched closely with empirical predictions based on total leg length (Fig. 13C). In terms of bone 
644 loading, all parameters also changed in a gradual fashion progressing from Daspletosaurus to the 645 chicken (Fig. 14 A,B). Thus, in Daspletosaurus, the femur was loaded predominantly in 646 mediolateral bending, whereas in the chicken the femur was loaded predominantly in torsion, 647 with bending predominantly in an anteroposterior direction. In 'Troodon', torsion was more 648 prominent compared to Daspletosaurus, but bending still remained the dominant loading regime. 649 As with the other parameters, muscular support also changed gradually progressing from 650 Daspletosaurus to the chicken Fig. 14C,D). In Daspletosaurus, the normalized hip abductor 651 moment was relatively high and the normalized hip medial rotator moment was relatively low, 652 whereas the situation was reversed in the chicken.

653

654

655

III.5 Discussion

656

657

Having previously demonstrated the validity and potential utility of the 'reverse' application of 658 the trajectorial theory (Part II; Bishop et al. in review-a), the aim of the present study was to 659 apply this approach to two extinct, non-avian theropods, Daspletosaurus torosus and 'Troodon' 660 (Troodontidae sp.), to gain new insight into their hindlimb locomotor biomechanics. In addition 661 to deriving a 'characteristic posture' for both species, quantitative results were produced that 662 have bearing on various questions concerning theropod locomotor biomechanics and its 663 evolution, posed in Section III.2. In particular, the evolutionary-biomechanical hypotheses of 664 Carrano (1998) and Hutchinson \& Gatesy (2000) were able to be quantitatively tested in a novel 665 way.

666

667

668

\section{III.5.1 Postures}

669

670

In the 'characteristic posture' identified for both non-avian theropods, there was generally a

672 strong alignment between calculated principal stress trajectories and observed patterns in cancellous bone architecture, across the femur, proximal tibia and proximal fibula. It is important to note that this should not be presumed to be the posture used by these extinct species at any

674 particular point in the stance phase; rather, the posture identified here is a time- and load- 
675 averaged characterization of the kinds of postures experienced on a daily basis. Nevertheless, 676 since the posture previously identified for the chicken corresponds well to the posture of a typical 677 avian hindlimb at around mid-stance in terrestrial locomotion (Part II), the postures derived for 678 Daspletosaurus and 'Troodon' are inferred to reflect the postures of these species at around the 679 mid-stance of normal locomotion. Thus, Daspletosaurus is inferred to have stood and moved 680 with a largely upright posture with a subvertical femoral orientation, whilst the limb posture of 681 'Troodon' is inferred to have been more crouched, although not to the degree observed in extant 682 birds. It is worth noting that the femoral orientation of the Daspletosaurus posture, in terms of the 683 degree of hip extension, is very similar to that hypothesized for other large, phylogenetically 684 basal tetanuran species by previous workers such as Tyrannosaurus (Gatesy et al. 2009; 685 Hutchinson 2004; Hutchinson et al. 2005), Allosaurus and Acrocanthosaurus (Bates et al. 2012). 686 The inferences drawn in those studies were based on the posture that allowed for high locomotor 687 forces to be sustained (Gatesy et al. 2009; Hutchinson 2004), or that achieved a maximal total 688 moment arm of the hip extensor muscles (Bates et al. 2012; Hutchinson et al. 2005). The 689 rationale of the latter set of studies is in some respects similar to the approach of the present study 690 (which used static optimization in the musculoskeletal modelling stage), in that both approaches 691 are dependent on the moment arms of individual muscles (see Part II, section II.5.1).

692

693

694

695

696

697

698

699

700

701

702

703

704

705

\section{III.5.2 Hip articulation in non-avian theropods}

The results of the exploratory analysis of hip articulations in the Daspletosaurus model supported the inference made in Part I of this series: in non-avian theropods such as Allosaurus and tyrannosaurids, the immediate articulation between the femur and acetabulum may have been centred about the apex of the femoral head. Other articulations, involving greater contribution from the cylindrical part of the femoral head lateral to the apex, did not result in as strong correspondence between principal stresses and cancellous bone architecture. This is not to say that these other articulations were not used during daily activity, rather that they may have been used less frequently. Indeed, as the entire proximal surface of the non-avian theropod femur 
706 typically bears a characteristic texture indicative of a hyaline cartilage covering (smooth on the 707 scale of millimetres, but wrinkled on the scale of centimetres; Tsai \& Holliday 2015; Tsai et al.

708 2018), this suggests that articulation between the lateral proximal femur and the incipient 709 antitrochanter on the ilium would have occurred on occasion, but the relatively frequency of this 710 remains unknown (see also Kambic et al. 2014; Kambic et al. 2015). This interpretation of hip 711 articulation is also consonant with anatomical considerations of the non-avian theropod pelvis 712 and sacrum. Specifically, a more lateral articulation of the (non-abducted) femur with the 713 acetabulum places the femoral head more medially with respect to the pelvis, which could bring 714 it into contact with the centra of the sacral vertebrae (e.g., Gilmore 1920; Osborn 1917; Rauhut \& 715 Carrano 2016).

716

717 Combined with the results of the exploratory analysis, the solution posture identified for the 718 Daspletosaurus model can help move toward resolving the question of how theropods with 719 proximomedially inclined femoral heads, such as tyrannosaurids and carcharodontosaurids, kept 720 their feet positioned close to the body midline, as indicated by fossil trackways (e.g., McCrea et 721 al. 2014). Previously, working on the assumption that the cylindrical part of the femoral head 722 articulated with the acetabulum, researchers had found that the femur inevitably becomes 723 markedly abducted from the body midline. Without further speculation about joint articulations 724 or the nature of the intervening soft tissues (cartilage, menisci) more distally in the limb, this 725 leads to an unnaturally wide foot placement (e.g., Bates et al. 2012; Hutchinson et al. 2005; 726 Hutchinson et al. 2007). Indeed, in the second variation of hip articulation tested for the

727

728

729

730

731

732

733

734

735

736

Daspletosaurus model, mediolateral step width was almost $47 \%$ of hip height (Fig. 3I), more than three times the typical step width observed in theropods (Bishop et al. 2017). With the hip articulation occurring at the apex of the femoral head, however, this allows for significant joint movement in other directions besides abduction-adduction. In particular, the solution posture identified for the Daspletosaurus model had a modest amount of external long-axis rotation, but little abduction of the femur; in fact, the femur was adducted slightly. Moreover, the asymmetry of the distal femoral condyles leads to a gently skewed orientation of the knee flexion-extension axis in the coronal plane, such that the distal crus is angled in towards the body midline (see Part II and Figs 1E and 2E). The combination of these features allows the pes to be positioned close to the midline, yet the upper limb be kept clear of the pelvis. 
Despite the potential that this new interpretation may have for understanding how non-avian

739

740

741

742

743

744

745

746

747

748

749

750

751

752

753

754

755

756

757

758

759

760

761

762

763

764

765

theropod hips may have articulated, it is worth emphasizing that it is based on a single posture, which at best can only be regarded as a snap shot of the limb during the stance phase of locomotion. A great deal more work is required if an understanding of dynamic joint articulations throughout the stride is to be achieved. One potential avenue is by using forward dynamic simulations (e.g., Sellers et al. 2017) to generate a variety of postures throughout the stance that may be used to inform musculoskeletal and finite element models. This would require more complex modelling of some joints than is currently done (e.g., three degrees of freedom for the hip), and would in turn require substantially greater computational power.

\section{III.5.3 Theropod locomotor evolution}

A second major objective of the current study was to test evolutionary-biomechanical hypotheses concerning posture, bone loading mechanics and muscular control strategies in theropods. In doing so, insight would be gained as to how such aspects of theropod locomotion may have evolved on the line to birds. As only three species have thus far been investigated, current assessments are necessarily coarse; yet, as these species span a broad part of the theropod family tree, this is sufficient to detect gross phyletic change in the aspects of locomotor biomechanics examined here. Indeed, that the results for the Daspletosaurus model are consistently quite different from those for the chicken model (Figs 12,13) is suggestive of pronounced evolutionary change between Coelurosauria and Neognathae.

The results for the three theropod species modelled here demonstrate that, progressing through theropod phylogeny towards more derived species, the following trends occurred:

1. The whole-body COM moved anteriorly; this was to be expected, given that model mass properties were largely derived from models developed in the study of Allen et al. (2013), who showed the same pattern. 
766 2. Hindlimb posture became more crouched, at least as far as the hip and knee joints are 767 concerned. This is consonant with the findings of previous work (Carrano 1998; Gatesy 768 1990; Gatesy 1991; Gatesy 1995).

7693 . Torsion became more prevalent than bending as the dominant loading regime of the femur.

770 4. The direction of bending of the femur changed from being predominantly mediolateral to 771 being predominantly anteroposterior.

772 5. Hip abduction became overtaken by hip long-axis rotation as the main muscular control 773 mechanism of stance-limb support.

774 For a given parameter, the value for 'Troodon' was intermediate between that for Daspletosaurus 775 and that for the chicken. This supports the hypothesis of a gradual evolutionary change in 776 locomotor biomechanics along the line to birds, but more taxa from different parts of theropod 777 phylogeny would need to be modelled to definitively rule out punctuated change at any point 778 along the stem lineage. Regardless of the mode of evolution of these parameters, the above 779 results do suggest that hindlimb posture, bone loading mechanics and muscular support strategies 780 were tightly associated with each other, supporting the hypotheses of Carrano (1998) and 781 Hutchinson \& Gatesy (2000). With the framework established in this series of studies, future 782 development of models for other species, from different theropod clades, will help further test 783 and clarify this interpretation.

784

The above trends identified in the present study are consilient with trends in other biomechanically relevant aspects, as noted by previous studies. These other trends include:

1. Modifications of pelvic and hindlimb osteology and musculature (Carrano 2000; Hutchinson 2001a; Hutchinson 2001b; Hutchinson 2002).

2. Decrease in tail length and prominence of caudofemoralis musculature (Gatesy 1990; Gatesy 1995; Gatesy 2002; Pittman et al. 2013).

3. A shift from caudofemoralis-mediated, hip-based limb retraction to 'hamstring'-mediated, knee-based limb retraction during gait (Gatesy 1990; Gatesy 1995; Gatesy 2002).

4. Changes in gross limb proportions, in particular a decrease in relative femur length, which in turn leads to an apparent increase in femoral diaphyseal robusticity (Carrano 1998; Gatesy \& Middleton 1997). 
796 5. The acquisition of a more continuous locomotor repertoire, where walking and running are 797 not discrete gaits (Bishop et al. 2017).

798 The timing of some of these changes remains uncertain (see also Hutchinson 2006), but it appears 799 that all were underway prior to the origin of Paraves (i.e., birds and their closest maniraptoran 800 relatives such as 'Troodon'), and that many, if not all, took place over a protracted period of time. 801

802 803 804 805 806 807 808

Most of the above changes also occurred in tandem with a progressive (Lee et al. 2014) or multistep (Benson et al. in press) reduction in body size along the theropod stem lineage. A decrease in body size - either along the theropod stem lineage, or by directly comparing Daspletosaurus, 'Troodon' and the chicken - might be expected in and of itself to bring about changes in posture, since posture correlates with body size in extant parasagittal tetrapods (Biewener 1989; Biewener 1990; Bishop et al. 2018; Gatesy \& Biewener 1991). However, since many other aspects of theropod anatomy and locomotor biomechanics also change in tandem with body size along the theropod stem lineage, it is presently not possible to disentangle the relative importance of body size (or any other single feature) on posture. That many aspects of theropod locomotor anatomy and biomechanics appear to have co-evolved over a protracted period of time, along with additional features such as forelimb enlargement (Allen et al. 2013; Dececchi \& Larsson 2013) and elaboration of forelimb integument (Xu et al. 2014; Zelenitsky et al. 2012), is an interesting phenomenon that warrants further investigation.

The results of this study may also have more general implications for understanding locomotor biomechanics (and its evolution) in tetrapod species that employ a largely parasagittal stance and gait. Previous in vivo strain gauge studies of parasagittal mammals that use a more crouched femoral posture have shown that the femur experiences a sizeable amount of torsional loading, in addition to bending (Butcher et al. 2011; Keller \& Spengler 1989). Additionally, finite element simulations of sit-to-stand and stand-to-sit behaviour in humans, behaviours that require limb support during crouched femoral orientations, have revealed a marked increase in torsional loading of the femur compared to normal locomotion (Villette 2016). In concert with the results of this study, these observations suggest that there is a continuum in musculoskeletal mechanics spanning from crouched to upright postures, of which birds and humans are 'end members'. In upright postures, hip abduction is the dominant mode of limb support, which results in bending 
827 being the dominant mode of loading of the femur. However, as the femur becomes more

828 crouched, the efficacy of hip abduction in providing limb support decreases, whilst that of hip

829 long-axis rotation increases; this in turn loads the femur in a greater degree of torsion (see also

830 Butcher et al. 2011).

831

832

833

III.5.4 Methodological considerations

834

835

A number of methodological considerations should be borne in mind when interpreting the

836 results of the present study. None are considered to be of any major importance for the main interpretations made here, but they do highlight areas where future research efforts could be 838 focused, potentially yielding further insight into theropod hindlimb biomechanics.

839

840

841

III.5.4.1 Correspondence in the distal tibiotarsus

842

843

It is worth re-iterating that little correspondence was evident between principal stresses and cancellous bone architecture in the distal parts of the tibiotarsus or fibula, in any posture tested

845 for all three theropod species modelled. Additionally, the architectural patterns observed in the fibular crest of tyrannosaurid tibiae could not be replicated in the Daspletosaurus model. As discussed in Part II, this could reflect an inadequate modelling formulation, adaptation of these parts of the bones to many varied loading regimes, or a combination of both (or other) factors. For the two extinct species at least, the normal in vivo loads experienced by the distal tibiotarsus may have also been influenced by the derived arctometatarsalian structure of their metatarsus (Holtz 1995; Snively \& Russell 2003; Wilson \& Currie 1985), a prospect requiring further investigation. Nevertheless, the architecture of cancellous bone in the distal tibiotarsus of theropods shows some strikingly different patterns between the various theropod groups. From a phenomenological perspective at least, this is indicative of marked differences in bone loading regimes, and by extension locomotor behaviour. It is therefore worthy of future modelling effort to establish a more mechanistic link between cancellous bone architecture and musculoskeletal loading mechanics in this part of the hindlimb. 
III.5.4.2 Pelvic orientation

861

862 One aspect of theropod posture that was not investigated in this study was the orientation of the 863 pelvis. In all simulations, the pelvis of the three theropod species modelled was oriented

864 865 866 867 868 869 similarly, with the sacral vertebrate oriented approximately horizontally and parallel to the $x$-axis of the global coordinate system. However, it is known that extant birds can employ significant amounts of pitch, roll or yaw during locomotion (Abourachid et al. 2011; Gatesy 1999a; Rubenson et al. 2007). If the pelvis underwent side-to-side rolling during locomotion in nonavian theropods, even by a small amount, this may have served to clear the pelvis and trunk further out of the way of the thigh of the stance leg. The effect of this would have been most obvious in species with well-developed pubic boots, such as large tyrannosaurids and allosauroids. Future investigation could therefore be directed towards incorporating one or more degrees of freedom in the pelvis segment of the models, as well as incorporating additional degrees of freedom in other joints (e.g., knee). Caution would need to be exercised, however, as the number of variable parameters could quickly grow to be very large, which may require a great deal more posture variations be tested before a 'solution posture' is satisfactorily obtained. However, as noted in Part II, the development of an automated optimization approach (in tandem with more extensive quantification of cancellous bone architecture) could allow for more degrees of freedom to be incorporated, and for more posture variations to be tested. This is a worthwhile avenue for future research, one that could make the reverse approach more easily applicable to a wider range of questions on tetrapod locomotor evolution.

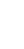

\section{III.5.4.3 Stresses in the medial femoral condyle}

As noted in the results of this study, as well as those of Part II, the mean direction of the minimum principal stress $\left(\boldsymbol{\sigma}_{3}\right)$ in the medial femoral condyle was notably more posteriorly 
889 inclined than the mean direction of the primary fabric orientation of cancellous bone $\left(\mathbf{u}_{1}\right)$, in both

890 the chicken and 'Troodon' models. This was the case regardless of the posture tested. The cause

891 for this discrepancy is probably twofold. Firstly, taking the mean direction of $\mathbf{u}_{1}$ in the medial

892 condyle will average out the 'fan' of individual fabric vectors (see Part I) that is ubiquitous in

893 theropods. Thus, there will be some parts of the condyle for which a greater correspondence

894 between fabric direction and the calculated principal stresses will indeed occur, namely, where

895 the fabric vectors are more posteriorly inclined than the overall orientation.

896

897 Secondly, it is quite possible that the individual $\mathbf{u}_{1}$ vectors throughout the medial condyle may

898 also 'reflect' the maximum principal stress $\left(\boldsymbol{\sigma}_{1}\right)$ in addition to $\boldsymbol{\sigma}_{3}$, and so do not fully align with

899 the calculated directions of either one. Given that motion of the theropod knee is inferred to have

900 predominantly occurred in the flexion-extension plane (but see Kambic et al. 2015), the main

901 loading regimes expected in the femoral condyles would be expected be anteroposteriorly

902 oriented, as also suggested by the 'butterfly pattern' of the secondary fabric direction in the

903 condyles (see Part I). Hence, both $\boldsymbol{\sigma}_{1}$ and $\boldsymbol{\sigma}_{3}$ could be expected to be largely constrained to a

904 parasagittal orientation, which could influence the direction of $\mathbf{u}_{1}$ throughout the medial condyle.

905

906

907 III.6 Conclusion

908

909 By applying the trajectorial theory in reverse, this study sought to identify a single, characteristic

910 posture for two extinct, non-avian theropods that can explain a considerable amount of the

911 architecture of cancellous bone observed in the hindlimb bones of these species. The postures

912 derived for Daspletosaurus torosus and 'Troodon' are inferred to reflect the postures used at

913 around mid-stance during normal terrestrial locomotion, but should not be presumed to have been

914 the postures used. The largely upright posture identified for Daspletosaurus is comparable to the

915 postures previously hypothesized for other large, phylogenetically basal tetanuran species of non-

916 avian theropod. The posture identified for 'Troodon' is more crouched than that of

917 Daspletosaurus, especially in regard to femoral orientation, but not to the degree observed in

918 extant birds. The results of this study also provide an alternative perspective on the manner of

919 articulation of the non-avian theropod hip joint, and suggest a solution to how non-avian 
920 theropods with proximomedially inclined femoral heads maintained narrow mediolateral foot

921 placements.

922

923 In addition to improving understanding of posture in non-avian theropods, this study provides a

924 new approach for how evolutionary-biomechanical hypotheses of locomotion can be explicitly

925 and quantitatively tested. By using a previously underexplored line of evidence, cancellous bone

926 architecture, the results of this study have supported the hypotheses of Carrano (1998) and

927 Hutchinson \& Gatesy (2000). Progressing from coelurosaurs through to extant birds, a number of

928 important changes are inferred to have occurred in concert with one another, involving whole-

929 body COM position, hindlimb posture, bone loading mechanics and muscular control strategies.

930 The pattern of the changes also supports a more gradual fashion of change (as opposed to more

931 punctuated), adding to the growing body of evidence suggesting that the unique locomotor

932 repertoire of extant birds was acquired over a long period of time. However, only three species

933 were modelled here, and so a more rigorous testing of the exact mode and tempo of evolutionary

934 change awaits the modelling of additional species.

935

936 The integrative biomechanical modelling approach developed in Part II provides useful insights

937 into non-avian theropod hindlimb locomotor biomechanics, as well as how this evolved along the

938 line to extant birds. The generality of the approach means that it could be useful for

939 understanding locomotor behaviour, and its evolution, in other extinct vertebrate groups as well.

940 Examples of future research that could apply the approach include: forelimb posture and use in

941 quadrupedal dinosaurs, such as ceratopsians (Fujiwara \& Hutchinson 2012; Johnson \& Ostrom

942 1995); the evolution of powered flight in birds, bats and pterosaurs (Bishop 2008; Heers \& Dial

943 2012; Thewissen \& Babcock 1992; Unwin 2005); the evolution of posture in synapsids on the

944 line to mammals (Blob 2001; Kemp 1982; Lai et al. 2018); and the evolution of terrestrial

945 locomotor capabilities in stem tetrapods (Clack 2012; Pierce et al. 2013). It may also prove to be

946 of use for questions of biomechanics not related to locomotion, such as the posture of sauropod

947 dinosaur necks (Stevens \& Parrish 2005; Taylor et al. 2009).

948

949 III.7 Acknowledgements

950 
951 The staff of the Geosciences Program of the Queensland Museum is thanked for the provision of

952 workspace and access to literature: A. Rozefelds, K. Spring, R. Lawrence, P. Tierney, J.

953 Wilkinson and D. Lewis. Much appreciation is extended to the staff and associated colleagues of

954 the institutions that provided access to the material studied here: D. Henderson, B. Strilisky, G.

955 Housego, R. Russel, T. Courtenay, B. Sanchez and F. Therrien (Royal Tyrell Museum of

956 Palaeontology, Drumheller); R. Irmis, C. Levitt-Bussian, C. Webb and P. Policelli (Natural

957 History Museum of Utah, Salt Lake City); J. Horner, J. Scannella, D. Varricchio, D. Strosnider,

958 C. Woodruff, D. Fowler and T. Carr (Museum of the Rockies, Bozeman). Many of the above

959 people also provided helpful discussion on various aspects of theropod biology, and also helped

960 transport specimens for CT scanning. Those who facilitated or performed the scanning itself are

961 also greatly thanked: S. Purdy and D. Wetter (Canada Diagnostic Centres, Calgary); K. Ugrin and

962 D. Van Why (Bozeman Deaconess Hospital, Bozeman); and S. Merchant, E. Hsu and J. Morgan

963 (HSC Cores Research Facility, University of Utah, Salt Lake City). The thorough and

964 constructive comments on earlier versions of the manuscript, provided by S. Gatesy, T. Ryan, D.

965 Henderson, E. Snively and an anonymous reviewer, are all greatly appreciated, and substantially

966 improved the clarity and content of the research presented here. All scripts and data used are held

967 in the Geosciences Collection of the Queensland Museum, and will be made available upon

968 request to the Collections Manager. Additionally, a complete copy of the fossil CT scan data

969 obtained in the present study is accessioned with the respective institutions in which the

970 specimens are housed.

971

972

\section{III.8 References}

974

975

976

977

978

979

980

981

982

983

984

Abourachid A, Hackert R, Herbin M, Libourel PA, Lambert F, Gioanni H, Provini P, Blazevic P, and Hugel V. 2011. Bird terrestrial locomotion as revealed by 3-D kinematics. Zoology 114:360-368.

Alexander RM. 1989. Dynamics of Dinosaurs and Other Extinct Giants. New York: Columbia University Press.

Allen V, Bates KT, Li Z, and Hutchinson JR. 2013. Linking the evolution of body shape and locomotor biomechanics in bird-line archosaurs. Nature 497:104-107.

Allen V, Paxton H, and Hutchinson JR. 2009. Variation in Center of Mass Estimates for Extant Sauropsids and its Importance for Reconstructing Inertial Properties of Extinct Archosaurs. The Anatomical Record 292:1442-1461. 
985

986

987

988

989

990

991

992

993

994

995

996

997

998

999

1000

1001

1002

1003

1004

1005

1006

1007

1008

1009

1010

1011

1012

1013

1014

1015

1016

1017

1018

1019

1020

1021

1022

1023

1024

1025

1026

1027

1028

1029

1030

Allmendinger RW, Cardozo NC, and Fisher D. 2013. Structural Geology Algorithms: Vectors and Tensors. Cambridge: Cambridge University Press.

Attene M, and Falcidieno B. 2006. ReMESH: An Interactive Environment to Edit and Repair Triangle Meshes. Proceedings of the Eighth International Conference on Shape Modeling and Applications. Matushima. p 271-276.

Bakker RT. 1986. The Dinosaur Heresies. New York: William Morrow \& Company, Inc.

Bates BT, and Schachner ER. 2012. Disparity and convergence in bipedal archosaur locomotion. Journal of the Royal Society Interface 9:1339-1353.

Bates KT, Benson RBJ, and Falkingham PL. 2012. A computational analysis of locomotor anatomy and body mass evolution in Allosauroidea (Dinosauria: Theropoda). Paleobiology 38:486-507.

Bates KT, Falkingham PL, Breithaupt BH, Hodgetts D, Sellers WI, and Manning PL. 2009a. How big was 'Big Al'? Quantifying the effect of soft tissue and osteological unknowns on mass predictions for Allosaurus (Dinosauria: Theropoda). Palaeontologia Electronica 12:14A.

Bates KT, Manning PL, Hodgetts D, and Sellers WI. 2009b. Estimating Mass Properties of Dinosaurs Using Laser Imaging and 3D Computer Modelling. PLoS ONE 4:e4532.

Beer FP, Johnston ER, Jr, DeWolf JT, and Mazurek DF. 2012. Mechanics of Materials. New York: McGraw-Hill.

Benson RBJ, Hunt G, Carrano MT, and Campione NE. in press. Cope's Rule and the adaptive landscape of dinosaur body size evolution. Palaeontology.

Biewener AA. 1989. Scaling Body Support in Mammals: Limb Posture and Muscle Mechanics. Science 245:45-48.

Biewener AA. 1990. Biomechanics of Mammalian Terrestrial Locomotion. Science 250:10971103.

Biewener AA. 1992. In vivo measurement of bone strain and tendon force. In: Biewener AA, ed. Biomechanics - Structures and Systems: A Practical Approach. New York: Oxford University Press.

Bishop KL. 2008. The evolution of flight in bats: narrowing the field of plausible hypotheses. Quarterly Review of Biology 83:153-169.

Bishop PJ, Clemente CJ, Graham DF, Lamas LP, Hutchinson JR, Rubenson J, Hancock JA, Wilson RS, Hocknull SA, Barrett RS, and Lloyd DG. 2018. The Influence of Speed and Size on Avian Terrestrial Locomotor Biomechanics: Predicting Locomotion in Extinct Theropod Dinosaurs. PLoS ONE 13:e0192172.

Bishop PJ, Clemente CJ, Weems RE, Graham DF, Lamas LP, Hutchinson JR, Rubenson J, Wilson RS, Hocknull SA, Barrett RS, and Lloyd DG. 2017. Using step width to compare locomotor biomechanics between extinct, non-avian theropod dinosaurs and modern obligate bipeds. Journal of the Royal Society Interface 14:20170276.

Bishop PJ, Hocknull SA, Clemente CJ, Hutchinson JR, Barrett RS, and Lloyd DG. in review-a. Cancellous bone architecture and theropod dinosaur locomotion. Part II - A new approach to reconstructing posture and locomotor biomechanics in extinct tetrapod vertebrates. PeerJ.

Bishop PJ, Hocknull SA, Clemente CJ, Hutchinson JR, Farke AA, Beck BR, Barrett RS, and Lloyd DG. in review-b. Cancellous bone architecture and theropod dinosaur locomotion. Part I - An examination of cancellous bone architecture in the hindlimb bones of theropods. PeerJ. 
1031

1032

1033

1034

1035

1036

1037

1038

1039

1040

1041

1042

1043

1044

1045

1046

1047

1048

1049

1050

1051

1052

1053

1054

1055

1056

1057

1058

1059

1060

1061

1062

1063

1064

1065

1066

1067

1068

1069

1070

1071

1072

1073

1074

1075

1076

Blob RW. 2001. Evolution of hindlimb posture in nonmammalian therapsids: biomechanical tests of paleontological hypotheses. Paleobiology 27:14-38.

Brochu CA. 2003. Osteology of Tyrannosaurus rex: insights from a nearly complete skeleton and high-resolution computed tomographic analysis of the skull. Society of Vertebrate Paleontology Memoir 7:1-138.

Brusatte SL, Norell MA, Carr TD, Erickson GM, Hutchinson JR, Balanoff AM, Bever GS, Choiniere JN, Makovicky PJ, and Xu X. 2010. Tyrannosaur Paleobiology: New Research on Ancient Exemplar Organisms. Science 329:1481-1485.

Butcher MT, White BJ, Hudzik NB, Gosnell WC, Parrish JHA, and Blob RW. 2011. In vivo strains in the femur of the Virginia opossum (Didelphis viginiana) during terrestrial locomotion: testing hypotheses of evolutionary shifts in mammalian bone loading and design. Journal of Experimental Biology 214:2631-2640.

Campione NE, Evans DC, Brown CM, and Carrano MT. 2014. Body mass estimation in nonavian bipeds using a theoretical conversion to quadruped stylopodial proportions. Methods in Ecology and Evolution 5:913-923.

Cardozo NC, and Allmendinger RW. 2013. Spherical projections with OSXStereonet. Computers \& Geosciences 51:193-205.

Carrano MT. 1998. Locomotion in non-avian dinosaurs: integrating data from hindlimb kinematics, in vivo strains, and bone morphology. Paleobiology 24:450-469.

Carrano MT. 2000. Homoplasy and the evolution of dinosaur locomotion. Paleobiology 26:489512.

Carrano MT. 2001. Implications of limb bone scaling, curvature and eccentricity in mammals and non-avian dinosaurs. Journal of Zoology 254:41-55.

Carrano MT, and Hutchinson JR. 2002. Pelvic and hindlimb musculature of Tyrannosaurus rex (Dinosauria: Theropoda). Journal of Morphology 253:207-228.

Christiansen P. 1998. Strength indicator values of theropod long bones, with comments on limb proportions and cursorial potential. Gaia 15:241-255.

Christiansen P. 1999. Long bone scaling and limb posture in non-avian theropods: evidence for differential allometry. Journal of Vertebrate Paleontology 19:666-680.

Clack JA. 2012. Gaining Ground: The Origin and Evolution of Tetrapods. Bloomington: Indiana University Press.

Dececchi TA, and Larsson HCE. 2013. Body and limb size dissociation at the origin of birds: uncoupling allometric constraints across a macroevolutionary transition. Evolution 67:2741-2752.

Delp SL, Anderson FC, Arnold AS, Loan P, Habib A, John CT, Guendelman E, and Thelen DG. 2007. OpenSim: Open-Source Software to Create and Analyze Dynamic Simulations of Movement. IEEE Transactions of Biomedical Engineering 54:1940-1950.

Farlow JO, Chapman RE, Breithaupt BH, and Matthews N. 2012. The Scientific Study of Dinosaur Footprints. In: Brett-Surman MK, Holtz TR, Jr, and Farlow JO, eds. The Complete Dinosaur. 2 ed. Bloomington: Indiana University Press, 712-759.

Farlow JO, Smith MB, and Robinson JM. 1995. Body mass, bone "strength indicator," and cursorial potential of Tyrannosaurus rex. Journal of Vertebrate Paleontology 15:713725.

Fujiwara S, and Hutchinson JR. 2012. Elbow joint aductor moment arm as an indicator of forelimb posture in extinct quadrupedal tetrapods. Proceedings of the Royal Society of London, Series B 279:2561-2570. 
1077 Gao C, Morschhauser EM, Varricchio DJ, Liu J, and Zhao B. 2012. A Second Soundly Sleeping 1078 Dragon: New Anatomical Details of the Chinese Troodontid Mei long with Implications

Gatesy SM. 1990. Caudofemoral musculature and the evolution of theropod locomotion. Paleobiology 16:170-186.

Gatesy SM. 1991. Hind Limb Scaling in Birds and Other Theropods: Implications for Terrestrial Locomotion. Journal of Morphology 209:83-96.

Gatesy SM. 1994. Neuromuscular Diversity in Archosaur Deep Dorsal Thigh Muscles. Brain, Behavior and Evolution 43:1-14.

Gatesy SM. 1995. Functional evolution of the hindlimb and tail from basal theropods to birds. In: Thomason JJ, ed. Functional Morphology in Vertebrate Paleontology. New York: Cambridge University Press, 219-234.

Gatesy SM. 1997. An Electromyographic Analysis of Hindlimb Function in Alligator During Terrestrial Locomotion. Journal of Morphology 234:197-212.

Gatesy SM. 1999a. Guineafowl Hindlimb Function I: Cineradiographic Analysis and Speed Effects. Journal of Morphology 240:115-125.

Gatesy SM. 1999b. Guineafowl Hindlimb Function II: Electromyographic Analysis and Motor Pattern Evolution. Journal of Morphology 240:127-142.

Gatesy SM. 2002. Locomotor Evolution on the Line to Modern Birds. In: Chiappe LM, and Witmer LM, eds. Mesozoic Birds: Above the Heads of the Dinosaurs. Berkeley: University of California Press, 432-447.

Gatesy SM, Bäker M, and Hutchinson JR. 2009. Constraint-based exclusion of limb poses for reconstructing theropod dinosaur locomotion. Journal of Vertebrate Paleontology 29:535-544.

Gatesy SM, and Biewener AA. 1991. Bipedal locomotion: effects of speed, size and limb posture in birds and humans. Journal of Zoology 224:127-147.

Gatesy SM, and Middleton KM. 1997. Bipedalism, flight, and the evolution of theropod locomotor diversity. Journal of Vertebrate Paleontology 17:308-329.

Gatesy SM, Middleton KM, Jenkins FA, Jr, and Shubin NH. 1999. Three-dimensional preservation of foot movements in Triassic theropod dinosaurs. Nature 399:141-144.

Gilmore CW. 1920. Osteology of the carnivorous Dinosauria in the United States National Museum, with special reference to the genera Antrodemus (Allosaurus) and Ceratosaurus. Bulletin of the United States National Museum 110:1-159.

Heers AM, and Dial KP. 2012. From extant to extinct: locomotor ontogeny and the evolution of avian flight. Trends in Ecology and Evolution 27:296-305.

Henderson DM. 1999. Estimating the masses and centers of mass of extinct animals by 3-D mathematical slicing. Paleobiology 25:88-106.

Henderson DM, and Snively E. 2003. Tyrannosaurus en pointe: allometry minimized rotational inertia of large carnivorous dinosaurs. Biology Letters 271:S57-S60.

Holtz TR, Jr. 1995. The arctometatarsalian pes, an unusual structure of the metatarsus of Cretaceous Theropoda (Dinosauria: Saurischia). Journal of Vertebrate Paleontology 14:480-519.

Horner JR, and Lessem D. 1993. The Complete T. rex. New York: Simon and Schuster, Inc. Hotton NH, III. 1980. An Alternative to Dinosaur Endothermy: The Happy Wanderers. In: Thomas RDK, and Olson EC, eds. A Cold Look at the Warm-Blooded Dinosaurs. Boulder: Westview Press, Inc., 311-350. 
1123

1124

1125

1126

1127

1128

1129

1130

1131

1132

1133

1134

1135

1136

1137

1138

1139

1140

1141

1142

1143

1144

1145

1146

1147

1148

1149

1150

1151

1152

1153

1154

1155

1156

1157

1158

1159

1160

1161

1162

1163

1164

1165

1166

1167

1168

Hutchinson JR. 2001a. The evolution of femoral osteology and soft tissues on the line to extant birds (Neornithes). Zoological Journal of the Linnean Society 131:169-197.

Hutchinson JR. 2001b. The evolution of pelvic osteology and soft tissues on the line to extant birds (Neornithes). Zoological Journal of the Linnean Society 131:123-168.

Hutchinson JR. 2002. The evolution of hindlimb tendons and muscles on the line to crown-group birds. Comparative Biochemistry and Physiology, Part A 133:1051-1086.

Hutchinson JR. 2004. Biomechanical Modeling and Sensitivity Analysis of Bipedal Running Ability. II. Extinct Taxa. Journal of Morphology 262:441-461.

Hutchinson JR. 2006. The evolution of locomotion in archosaurs. Comptes Rendus Palevol 5:519-530.

Hutchinson JR, and Allen V. 2009. The evolutionary continuum of limb function from early theropods to birds. Naturwissenschaften 96:423-448.

Hutchinson JR, Anderson FC, Blemker SS, and Delp SL. 2005. Analysis of hindlimb muscle moment arms in Tyrannosaurus rex using a three-dimensional musculoskeletal computer model: implications for stance, gait, and speed. Paleobiology 31:676-701.

Hutchinson JR, Bates KT, Molnar J, Allen V, and Makovicky PJ. 2011. A Computational Analysis of Limb and Body Dimensions in Tyrannosaurus rex with Implications for Locomotion, Ontogeny and Growth. PLoS ONE 6:e26037.

Hutchinson JR, and Garcia M. 2002. Tyrannosaurus was not a fast runner. Nature 415:10181021.

Hutchinson JR, and Gatesy SM. 2000. Adductors, abductors, and the evolution of archosaur locomotion. Paleobiology 26:734-751.

Hutchinson JR, and Gatesy SM. 2006. Dinosaur locomotion: Beyond the bones. Nature 440:292294.

Hutchinson JR, Miller CE, Fritsch G, and Hildebrandt T. 2008. The Anatomical Foundation for Multidisciplinary Studies of Animal Limb Function: Examples from Dinosaur and Elephant Limb Imaging Studies. In: Endo H, and Frey R, eds. Anatomical Imaging: Towards a New Morphology. Tokyo: Springer, 23-38.

Hutchinson JR, Ng-Thow-Hing V, and Anderson FC. 2007. A 3D interactive method for estimating body segmental parameters in animals: Application to the turning and running performance of Tyrannosaurus rex. Journal of Theoretical Biology 246:660-6800.

Jacobson RD, and Hollyday M. 1982. A Behavioural and Electromyographic Study of Walking in the Chick. Journal of Neurophysiology 48:238-256.

Johnson RE, and Ostrom JH. 1995. The forelimb of Torosaurus and an analysis of the posture and gait of ceratopsian dinosaurs. In: Thomason JJ, ed. Functional Morphology in Vertebrate Paleontology. Cambridge: Cambridge University Press, 205-218.

Kambic RE, Roberts TJ, and Gatesy SM. 2014. Long-axis rotation: a missing degree of freedom in avian bipedal locomotion. Journal of Experimental Biology 217:2770-2782.

Kambic RE, Roberts TJ, and Gatesy SM. 2015. Guineafowl with a twist: asymmetric limb control in steady bipedal locomotion. Journal of Experimental Biology 218:3836-3844.

Keller TS, and Spengler DM. 1989. Regulation of bone stress and strain in the immature and mature rat femur. Journal of Biomechanics 22:1115-1127.

Kemp TS. 1982. Mammal-like Reptiles and the Origin of Mammals. London: Academic Press.

Lai PH, Biewener AA, and Pierce SE. 2018. Three-dimensional mobility and muscle attachments in the pectoral limb of the Triassic cynodont Massetognathus pascuali (Romer, 1967). Journal of Anatomy 232:383-406. 
1169

1170

1171

1172

1173

1174

1175

1176

1177

1178

1179

1180

1181

1182

1183

1184

1185

1186

1187

1188

1189

1190

1191

1192

1193

1194

1195

1196

1197

1198

1199

1200

1201

1202

1203

1204

1205

1206

1207

1208

1209

1210

1211

1212
Lambe LM. 1917. The Cretaceous theropodous dinosaur Gorgosaurus. Memoirs of the Geological Survey of Canada 100:1-84.

Lautenschlager S. 2016. Reconstructing the past: methods and techniques for the digital restoration of fossils. Royal Society Open Science 3:160342.

Lee MSY, Cau A, Naish D, and Dyke GJ. 2014. Sustained miniaturization and anatomical innovation in the dinosaurian ancestors of birds. Science 345:562-566.

Marsh RL, Ellerby DJ, Carr JA, Henry HT, and Buchanan CI. 2004. Partitioning the Energetics of Walking and Running: Swinging the Limbs is Expensive. Science 303:80-83.

Martelli S, Taddei F, Testi D, Delp SL, and Viceconti M. 2011. NMSBuilder: an application to personalize NMS models. Proceedings of the 23rd Congress of the International Society of Biomechanics. Brussels.

McCrea RT, Buckley LG, Farlow JO, Lockley MG, Currie PJ, Matthews NA, and Pemberton SG. 2014. A 'Terror of Tyrannosaurs': The First Trackways of Tyrannosaurids and Evidence of Gregariousness and Pathology in Tyrannosauridae. PLoS ONE 9:e103613.

Molnar RE, and Farlow JO. 1990. Carnosaur Paleobiology. In: Weishampel DB, Dodson P, and Osmólska H, eds. The Dinosauria. 1 ed. Berkeley: University of California Press, 210224.

Norell MA, and Makovicky PJ. 1997. Important Features of the Dromaeosaur Skeleton: Information from a New Specimen. American Museum Novitates 3215:1-28.

Norell MA, and Makovicky PJ. 1999. Important Features of the Dromaeosaurid Skeleton II: Information from Newly Collected Specimens of Velociraptor mongoliensis. American Museum Novitates 3282:1-45.

Osborn HF. 1917. Skeletal adaptations of Ornitholestes, Struthiomimus, Tyrannosaurus. Bulletin of the American Museum of Natural History 35:733-771.

Ostrom JH. 1969. Osteology of Deinonychus antirrhopus, and unusual theropod from the Lower Cretaceous of Montana. Bulletin of the Peabody Museum of Natural History 30:1-165.

Paul GS. 1998. Limb design, function and running performance in ostrich-mimics and tyrannosaurs. Gaia 15:257-270.

Pauwels F. 1980. Biomechanics of the Locomotor Apparatus. Berlin: Springer-Verlag.

Pierce SE, Hutchinson JR, and Clack JA. 2013. Historical Perspectives on the Evolution of Tetrapodomorph Movement. Integrative and Comparative Biology 53:209-223.

Pittman M, Gatesy SM, Upchurch P, Goswani A, and Hutchinson JR. 2013. Shake a Tail Feather: The Evolution of the Theropod Tail into a Stiff Aerodynamic Surface. PLoS ONE 8:e63115.

Rauhut OWM, and Carrano MT. 2016. The theropod dinosaur Elaphrosaurus bambergi Janensch, 1920, from the Late Jurassic of Tendaguru, Tanzania. Zoological Journal of the Linnean Society 178:546-610.

Reilly SM, and Blob RW. 2003. Motor control of locomotor hindlimb posture in the American alligator (Alligator mississippiensis). Journal of Experimental Biology 206:4327-4340.

Roberts TJ, Chen MS, and Taylor CR. 1998. Energetics of bipedal running. II. Limb design and running mechanics. Journal of Experimental Biology 205:2753-2762.

Rubenson J, Lloyd DG, Besier TF, Heliams DB, and Fournier PA. 2007. Running in ostriches (Stuthio camelus): three-dimensional joint axes alignment and joint kinematics. Journal of Experimental Biology 210:2548-2562. 
1213

1214

1215

1216

1217

1218

1219

1220

1221

1222

1223

1224

1225

1226

1227

1228

1229

1230

1231

1232

1233

1234

1235

1236

1237

1238

1239

1240

1241

1242

1243

1244

1245

1246

1247

1248

1249

1250

1251

1252

1253

1254

1255

1256

Sellers WI, and Manning PL. 2007. Estimating dinosaur maximum running speeds using evolutionary robotics. Proceedings of the Royal Society of London, Series B 274:27112716.

Sellers WI, Pond SB, Brassey CA, Manning PL, and Bates KT. 2017. Investigating the running abilities of Tyrannosaurus rex using stress-constrained multibody dynamic analysis. PeerJ 5:e3420.

Snively E, and Russell AP. 2003. Kinematic Model of Tyrannosaurid (Dinosauria: Theropoda) Arctometatarsus Function. Journal of Morphology 255:215-227.

Stevens KA, and Parrish JM. 2005. Digital Reconstructions of Sauropod Dinosaurs and Implications for Feeding. In: Curry Rogers KA, and Wilson JA, eds. The Sauropods: Evolution and Paleobiology. Berkeley: University of California Press, 178-200.

Taylor MP, Wedel MJ, and Naish D. 2009. Head and neck posture in sauropod dinosaurs inferred from extant animals. Acta Palaeontologica Polonica 54:213-220.

Thewissen JGM, and Babcock SK. 1992. The Origin of Flight in Bats. BioScience 42:340-345.

Thulborn T. 1990. Dinosaur Tracks. London: Chapman and Hall.

Tsai HP, and Holliday CM. 2015. Articular Soft Tissue Anatomy of the Archosaur Hip Joint: Structural Homology and Functional Implications. Journal of Morphology 276:601-630.

Tsai HP, Middleton KM, Hutchinson JR, and Holliday CM. 2018. Hip joint articular soft tissues of non-dinosaurian Dinosauromorpha and early Dinosauria: evolutionary and biomechanical implications for Saurischia. Journal of Vertebrate Paleontology 38:e1427593.

Tsuihiji T, Barsbold R, Watabe M, Tsogtbaatar K, Chinzorig T, Fujiyama Y, and Suzuki S. 2014. An exquisitely preserved troodontid theropod with new information on the palatal structure from the Upper Cretaceous of Mongolia. Naturwissenschaften 101:131-142.

Unwin DM. 2005. The Pterosaurs: From Deep Time. New York: Pi Press.

Valente G, Pitto L, Testi D, Seth A, Delp SL, Stagni R, Viceconti M, and Taddei F. 2014. Are Subject-Specific Musculoskeletal Models Robust to the Uncertainties in Parameter Identification? PLoS ONE 9:e112625.

van der Reest AJ, and Currie PJ. 2017. Troodontids (Theropoda) from the Dinosaur Park Formation, Alberta, with a description of a unique new taxon: implications for deinonychosaur diversity in North America. Canadian Journal of Earth Sciences 54:919935.

Villette CC. 2016. Structural Meso and Microscale Finite Element Based Approaches for the prediction of Bone Architecture and Fracture PhD. Imperial College London.

Wall-Scheffler CM, Chumanov E, Steudel-Numbers K, and Heiderscheit B. 2010. Electromyography Activity Across Gait and Incline: The Impact of Muscular Activity on Human Morphology. American Journal of Physical Anthropology 143:601-611.

Wilson MC, and Currie PJ. 1985. Stenonychosaurus inequalis (Saurischia: Theropoda) from the Judith River (Oldman) Formation of Alberta: new findings on metatarsal structure. Canadian Journal of Earth Sciences 22:1813-1817.

Xu X, Norell MA, Wang X, Makovicky PJ, and Wu X. 2002. A basal troodontid from the Early Cretaceous of China. Nature 415:780-784.

Xu X, Zhou Z, Dudley R, Mackem S, Chuong C-M, Erickson GM, and Varricchio DJ. 2014. An integrative approach to understanding bird origins. Science 346:1253293. 
1257

1258

1259

1260

1261

\section{III.9 Figure captions}

\section{3}

1264

1265

1266

1267

1268

1269

1270

1271

1272

1273

1274

1275

1276

1277

1278

1279

1280

1281

1282

1283

1284

1285

1286

Zelenitsky DK, Therrien F, Erickson GM, DeBuhr CL, Kobayashi Y, Eberth DA, and Hadfield F. 2012. Feathered Non-Avian Dinosaurs from North America Provide Insight into Wing Origins. Science 338:510-514.

Figure 1. The musculoskeletal model of the Daspletosaurus hindlimb developed in this study. This is shown in the 'neutral posture' for all joints, that is, when all joint angles are zero. (A-C) Geometries of the musculotendon actuators in relation to the bones, in lateral (A), anterior (B) and oblique anterolateral (C) views. (D-F) Location and orientation of joint coordinate systems (red, green and blue axes), the centres of mass for each segment (grey and white balls) and the soft tissue volumes used to calculate mass properties; these are shown in the same views as A-C. Also reported in D are the masses for each segment; the pelvis segment represents the body as well as the contralateral limb. In D-F, the flexion-extension axis of each joint is the blue axis. For scale, the length of each arrow in the triad of the global coordinate system is $500 \mathrm{~mm}$.

Figure 2. The musculoskeletal model of the 'Troodon' hindlimb developed in this study. This is shown in the neutral posture for all joints. A-C, geometries of the musculotendon actuators in relation to the bones, in lateral (A), anterior (B) and oblique anterolateral (C) views. (D-F) Location and orientation of joint coordinate systems (red, green and blue axes), the centres of mass for each segment (grey and white balls) and the soft tissue volumes used to calculate mass properties; these are shown in the same views as A-C. Also reported in D are the masses for each segment; the pelvis segment represents the body as well as the contralateral limb. In D-F, the flexion-extension axis of each joint is the blue axis. For scale, the length of each arrow in the triad of the global coordinate system is $200 \mathrm{~mm}$. 
1287 Figure 3. Varying the articulation of the hip joint in the Daspletosaurus model. (A-C) The 1288 original 'solution posture' identified for the Daspletosaurus model. (D-F) The first variation in 1289 hip articulation, where the femur (and limb distal to it) is moved medially by $50 \mathrm{~mm}$. (G-I) The 1290 second variation in hip articulation, where the femur (and limb distal to it) is moved medially by $129150 \mathrm{~mm}$, also with a sizeable amount of hip abduction and external long-axis rotation. A, D and G 1292 are in oblique anterolateral view; B, E and $\mathrm{H}$ are in close-ups of the hip articulation in anterior 1293 view; C, F and I show the whole hindlimb in anterior view, to illustrate the effect of differing hip 1294 articulations on gross limb position. Intervening soft tissues used in the finite element simulations are shown in turquoise; for clarity, the ilium and pubis are shown translucent in B, E and H. Also illustrated in B are the relative diameters of the femoral head (solid lines) and the acetabulum (dashed lines).

Figure 4. The postures tested for in Daspletosaurus. Around the periphery are the different postures tested, shown in lateral view, with the final solution posture in the centre box, shown in lateral, dorsal and anterior views; the whole-body COM location is also shown for the solution posture in lateral view. Joint angles for each posture are given in blue font; hip joint angles are given in the order of flexion-extension, abduction-adduction and long-axis rotation. Hip extension angle is expressed relative to the horizontal, whereas knee and ankle angles are expressed relative to the femur and tibiotarsus (respectively). For the other hip angles, positive values indicate abduction and external rotation, whereas negative values indicate adduction and internal rotation. The metatarsophalangeal joint angle is expressed relative to the neutral posture. The angular deviation between $\boldsymbol{\sigma}_{3}$ and $\mathbf{u}_{1}$ for each posture is also given in red font (reported as femoral head, then medial femoral condyle). The solution posture resulted in the greatest degree of overall correspondence between principal stress trajectories and observed cancellous bone architectural patterns in birds, as assessed by qualitative comparisons across the femur, tibiotarsus and fibula, as well as quantitative results for the femoral head and medial femoral condyle. 
1317 Figure 5. The postures tested for in 'Troodon'. Around the periphery are the different postures 1318 tested, shown in lateral view, with the final solution posture in the centre box, shown in lateral, 1319 dorsal and anterior views; the whole-body COM location is also shown for the solution posture in 1320 lateral view. Joint angles for each posture are given in blue font, following the same conventions 1321 as Fig. 4. The angular deviation between $\boldsymbol{\sigma}_{3}$ and $\mathbf{u}_{1}$ for each posture is also given in red font 1322 (reported as femoral head, then medial femoral condyle). The solution posture resulted in the 1323 greatest degree of overall correspondence between principal stress trajectories and observed 1324 cancellous bone architectural patterns in birds, as assessed by qualitative comparisons across the femur, tibiotarsus and fibula, as well as quantitative results for the femoral head and medial femoral condyle.

Figure 6. Principal stress trajectories for the proximal femur in the solution posture of Daspletosaurus, compared with observed cancellous bone fabric. For easier visual comparison, the stress trajectories were 'downsampled' in a custom MATLAB script, by interpolating the raw stress results at each finite element node to a regular grid. (A) Vector field of $\boldsymbol{\sigma}_{1}$ (red) and $\boldsymbol{\sigma}_{3}$ (blue) in a 3-D slice through the proximal femur, parallel to the coronal plane and through the middle of the femoral head, in anterior view. Note how the trajectory of $\boldsymbol{\sigma}_{3}$ projects towards the apex of the femoral head (green braces). (B) Observed cancellous bone architecture in the proximal femur of Allosaurus and tyrannosaurids (cf. Part I), in the same view as A. (C) Vector field of $\sigma_{1}$ and $\sigma_{3}$ in a 3-D slice through the lesser trochanter, parallel to the plane of the trochanter, in anterolateral view. (D) Observed cancellous bone architecture in the lesser trochanter of Allosaurus and tyrannosaurids (cf. Part I), in the same view as C. (E) Vector field of $\boldsymbol{\sigma}_{3}$ in the femoral head, shown as a 3-D slice parallel to the sagittal plane and through the apex of the head, in medial view. (F) Observed cancellous bone architecture in the femoral head of Allosaurus and tyrannosaurids (cf. Part I), in the same view as E. (G) Comparison of the mean direction of $\boldsymbol{\sigma}_{3}$ in the femoral head (blue) and the estimated mean direction of $\mathbf{u}_{1}$ for Allosaurus and tyrannosaurids (red), plotted on an equal-angle stereoplot with northern hemisphere projection (using StereoNet 9.5; Allmendinger et al. 2013; Cardozo \& Allmendinger 2013). Inset shows location of region for which the mean direction of $\boldsymbol{\sigma}_{3}$ was calculated. 
1349 Figure 7. Principal stress trajectories for the distal femur and fourth trochanter in the solution

1350

1351

1352

1353

1354

1355

1356

1357

1358

1359

1360

1361

1362

1363

1364

1365

1366

1367

1368

1369

1370

1371

1372

1373

1374

1375

1376

1377

1378

posture of Daspletosaurus, compared with observed cancellous bone fabric. (A) Vector field of

$\boldsymbol{\sigma}_{1}(\mathrm{red})$ and $\boldsymbol{\sigma}_{3}$ (blue) in a 3-D slice, parallel to the coronal plane and through the anterior aspect of the distal metaphysis, in anterior view. (B) Observed cancellous bone architecture in the distal metaphysis of Allosaurus and tyrannosaurids (cf. Part I), in the same view as A. (C) Vector field of $\sigma_{1}$ in the fourth trochanter, in medial view. (D) Observed cancellous bone architecture in the fourth trochanter of Allosaurus and tyrannosaurids (cf. Part I), in the same view as C. (E) Vector field of $\sigma_{3}$ in the lateral condyle, shown as a 3-D slice parallel to the sagittal plane and through the middle of the condyle. (F) Observed cancellous bone architecture in the lateral condyle of Allosaurus and tyrannosaurids (cf. Part I), in the same view as E. (G) Vector field of $\boldsymbol{\sigma}_{3}$ in the medial condyle, shown as a 3-D slice parallel to the sagittal plane and through the middle of the condyle. (H) Observed cancellous bone architecture in the medial condyle of Allosaurus and tyrannosaurids (cf. Part I), in the same view as G. (I) Comparison of the mean direction of $\boldsymbol{\sigma}_{3}$ in the medial condyle (blue) and the estimated mean direction of $\mathbf{u}_{1}$ for Allosaurus and tyrannosaurids (red), plotted on an equal-angle stereoplot with southern hemisphere projection. Inset shows location of region for which the mean direction of $\boldsymbol{\sigma}_{3}$ was calculated.

Figure 8. Principal stress trajectories for the tibia and fibula in the solution posture for Daspletosaurus, compared with observed cancellous bone fabric. (A) Vector field of $\boldsymbol{\sigma}_{3}$ in the medial tibial condyle, shown as a 3-D slice through the middle of the condyle and parallel to the sagittal plane, in medial view. (B) Observed cancellous bone architecture in the medial tibial condyle of Allosaurus and tyrannosaurids (cf. Part I), in the same view as A. (C) Vector field of $\boldsymbol{\sigma}_{3}$ in the medial and lateral tibial condyles, shown as 3-D slices through the middle of the condyles and parallel to the coronal plane, in posterior view. (D) Observed cancellous bone architecture in the medial and lateral tibial condyles of Allosaurus and tyrannosaurids (cf. Part I), in the same view as C. (E) Vector field of $\sigma_{3}$ in the lateral tibial condyle, shown as a 3-D slice through the middle of the condyle and parallel to the sagittal plane, in lateral view. (F) Observed cancellous bone architecture in the lateral tibial condyle of Allosaurus and tyrannosaurids (cf. Part I), in the same view as E. (G) Vector field of $\boldsymbol{\sigma}_{1}$ in the cnemial crest, shown as a 3-D slice 
1379 parallel to the coronal plane, in anterior view. $(\mathrm{H})$ Observed cancellous bone architecture in 1380 cnemial crest of Allosaurus and tyrannosaurids (cf. Part I), sectioned in the plane of the crest, 1381 shown in the same view as G; blue section lines illustrate primary architectural direction. (I) 1382 Vector field of $\sigma_{1}$ in the cnemial crest, shown as a 3-D slice parallel to the sagittal plane, in 1383 medial view. (J) Observed cancellous bone architecture in cnemial crest of Allosaurus and 1384 tyrannosaurids (cf. Part I), sectioned in the plane of the crest, shown in the same view as I. (K) Vector field of $\boldsymbol{\sigma}_{3}$ in the medial aspect of the fibular head, in medial view. (L) Observed cancellous bone architecture in the fibular head of Allosaurus and tyrannosaurids (cf. Part I), in 1387 the same view as $\mathrm{K}$.

1388

1389

1390

1391

1392

Figure 9. Principal stress trajectories for the proximal femur in the solution posture of 'Troodon', compared with observed cancellous bone fabric. (A, B) Vector field of $\boldsymbol{\sigma}_{3}$ in the femoral head, shown as 3-D slices parallel to the coronal plane (A, in anterior view) and sagittal plane (B, in 1393 medial view). (C, D) Observed vector field of $\mathbf{u}_{1}$ in the femoral head, in the same views as $A$ and B, respectively (cf. Part I). (E) Comparison of the mean direction of $\boldsymbol{\sigma}_{3}$ in the femoral head (blue) and the mean direction of $\mathbf{u}_{1}$ (red), plotted on an equal-angle stereoplot with northern hemisphere projection. Inset shows location of region for which the mean direction of $\boldsymbol{\sigma}_{3}$ was calculated. (F, G) Vector field of $\boldsymbol{\sigma}_{3}$ under the greater trochanter, shown as 3-D slices parallel to the coronal plane $(F$, in posterior view) and sagittal plane (G, in lateral view). (H, I) Observed vector field of $\mathbf{u}_{1}$ under the greater trochanter, shown in the same views as F and G, respectively (cf. Part I). (J) Vector field of $\boldsymbol{\sigma}_{1}$ in the lesser trochanter, shown in oblique anterolateral view. (K) Observed vector field of $\mathbf{u}_{1}$ in the lesser trochanter, shown in the same view as $\mathrm{J}$ for both specimens studied (cf. Part I).

Figure 10. Principal stress trajectories for the distal femoral condyles in the solution posture of 1406 'Troodon', compared with observed cancellous bone fabric. (A) Vector field of $\sigma_{3}$ in the lateral condyle, shown as a 3-D slice parallel to the sagittal plane. (B) Observed vector field of $\mathbf{u}_{1}$ in the lateral condyle, shown in the same view as A (cf. Part I). (C) Vector field of $\sigma_{3}$ in the medial condyle, shown as a 3-D slice parallel to the sagittal plane. (D) Observed vector field of $\mathbf{u}_{1}$ in the 
1410 medial condyle, shown in the same view as C (cf. Part I). (E) Comparison of the mean direction 1411 of $\boldsymbol{\sigma}_{3}$ in the medial condyle (blue) and the mean direction of $\mathbf{u}_{1}$ (red), plotted on an equal-angle 1412 stereoplot with southern hemisphere projection. This shows that in the solution posture the mean 1413 direction of $\boldsymbol{\sigma}_{3}$ was of the same general azimuth as the mean direction of $\mathbf{u}_{1}$, but was markedly 1414 more posteriorly inclined. Inset shows location of region for which the mean direction of $\boldsymbol{\sigma}_{3}$ was 1415 calculated.

Figure 11. Principal stress trajectories for the tibia and fibula in the solution posture for 'Troodon', compared with observed cancellous bone fabric. (A) Vector field of $\sigma_{3}$ in the medial tibial condyle, shown as a 3-D slice through the middle of the condyle and parallel to the sagittal plane, in medial view. (B) Observed vector field of $\mathbf{u}_{1}$ in the medial tibial condyle, in the same view as A (cf. Part I). (C) Vector field of $\boldsymbol{\sigma}_{3}$ in the medial and lateral tibial condyles, shown as 3D slices through the middle of the condyles and parallel to the coronal plane, in posterior view. (D) Observed vector field of $\mathbf{u}_{1}$ in the medial and lateral tibial condyles, in the same view as $\mathrm{C}$ (cf. Part I). (E) Vector field of $\boldsymbol{\sigma}_{3}$ in the lateral tibial condyle, shown as a 3-D slice through the middle of the condyle and parallel to the sagittal plane, in lateral view. (F) Observed vector field of $\mathbf{u}_{1}$ in the lateral tibial condyle, in the same view as E (cf. Part I). (G) Vector field of $\boldsymbol{\sigma}_{1}$ in the cnemial crest, shown as a 3-D slice parallel to the coronal plane, in anterior view. $(\mathrm{H})$ Observed vector field of $\mathbf{u}_{1}$ in the cnemial crest, in the same view as G (cf. Part I). (I) Vector field of $\boldsymbol{\sigma}_{1}$ in the cnemial crest, shown as a 3-D slice parallel to the sagittal plane, in medial view. (J) Observed vector field of $\mathbf{u}_{1}$ in the cnemial crest, in the same view as I (cf. Part I). (K) Vector field of $\boldsymbol{\sigma}_{1}$ in the lateral fibular head, in lateral view. (L) Vector field of $\boldsymbol{\sigma}_{3}$ in the medial fibular head, in medial view (reversed). (M) Observed vector field of $\mathbf{u}_{1}$ in the fibular head, in the same view as K (cf. Part I).

Figure 12. Principal stress trajectories for the proximal femur of Daspletosaurus in the two variations in hip articulation tested. (A) Vector field of $\sigma_{3}$ in the first variation tested, shown as a 3-D slice parallel to the coronal plane and through the middle of the femoral head. (B) Vector field of $\sigma_{3}$ in the first variation tested, shown as a 3-D slice parallel to the sagittal plane and 
1441 through the apex of the femoral head. (C) Vector field of $\sigma_{3}$ in the second variation tested, shown

1442 as a 3-D slice parallel to the coronal plane and through the middle of the femoral head. (D)

1443 Vector field of $\sigma_{3}$ in the second variation tested, shown as a 3-D slice parallel to the sagittal plane

1444 and through the apex of the femoral head. A and C are in anterior view, B and D are in medial

1445 view. Note in particular how the trajectory of $\sigma_{3}$ projects towards the more cylindrical part of the 1446 femoral head, lateral to the apex (green braces); compare to Fig. 6A,B,E,F. Also note in C how $\sigma_{3}$

1447 has a strong medial component near the apex of the head.

Figure 13. Comparison of parameters related to posture, extracted from the solution postures of the three species modelled: Daspletosaurus ('D'), 'Troodon' ('T') and the chicken ('C'). (A) Schematic illustration of the solution postures obtained for the three species, along with the location of the whole-body centre of mass (black and white disc). (B) Whole-body centre of mass location anterior to the hips, normalized to total leg length. (C) Degree of crouch for each species, both as measured from the solution posture, as well as empirically predicted from the data reported by Bishop et al. (2018). (D) Angles of the hip and knee joints. The hip extension angle is expressed relative to the horizontal, whereas the knee flexion angle is expressed relative to the femur. (E) Long-axis rotation and adduction-abduction of the hip joint. Positive values indicate external rotation and abduction (respectively), whereas negative values indicate internal rotation and adduction (respectively).

Figure 14. Comparison of parameters related to bone loading mechanics and muscular support, extracted from the solution postures of the three species modelled: Daspletosaurus ('D'), 'Troodon' ('T') and the chicken ('C'). (A) Orientation of the neutral surface of bending and the orientation of principal stresses $\left(\boldsymbol{\sigma}_{1}\right.$ and $\left.\boldsymbol{\sigma}_{3}\right)$ relative to the femur long-axis, both measured at midshaft. Insets show the neutral surface with respect to the mid-shaft cross-section, as well as anatomical directions ('A', anterior; 'P', posterior; 'M', medial; 'L', lateral). (B) Ratio of maximum shear to bending stress in the femoral mid-shaft. (C) Normallized moments of hip abductor and medial rotator muscles. The hip abductor for all species is the iliofemoralis externus (activation set to zero in the chicken; see Part II). In Daspletosaurus and 'Troodon', the medial 
1472 rotators are the iliotrochantericus caudalis and puboischiofemorales internus 1 et 2; in the 1473 chicken, they are the iliotrochanterici caudalis et medius. (D) Oblique anterolateral view of the 1474 hip of Daspletosaurus, showing the abductor and medial rotator muscles (colours as in C). 


\section{Table $\mathbf{1}$ (on next page)}

The specimens utilized in building the models of Daspletosaurus torosus and ' Troodon '.

Also listed are the settings used in acquiring CT scans; the geometry of specimens that were not CT scanned was captured via digital photogrammetry.

*Collection number abbreviations: MOR, Museum of the Rockies; TMP, Royal Tyrrell Museum of Palaeontology; UMNH VP; Natural History Museum of Utah. 
Table 1. The specimens utilized in building the models of Daspletosaurus torosus and 'Troodon'. Also listed are the settings used in acquiring CT scans; the geometry of specimens that were not CT scanned was captured via digital photogrammetry.

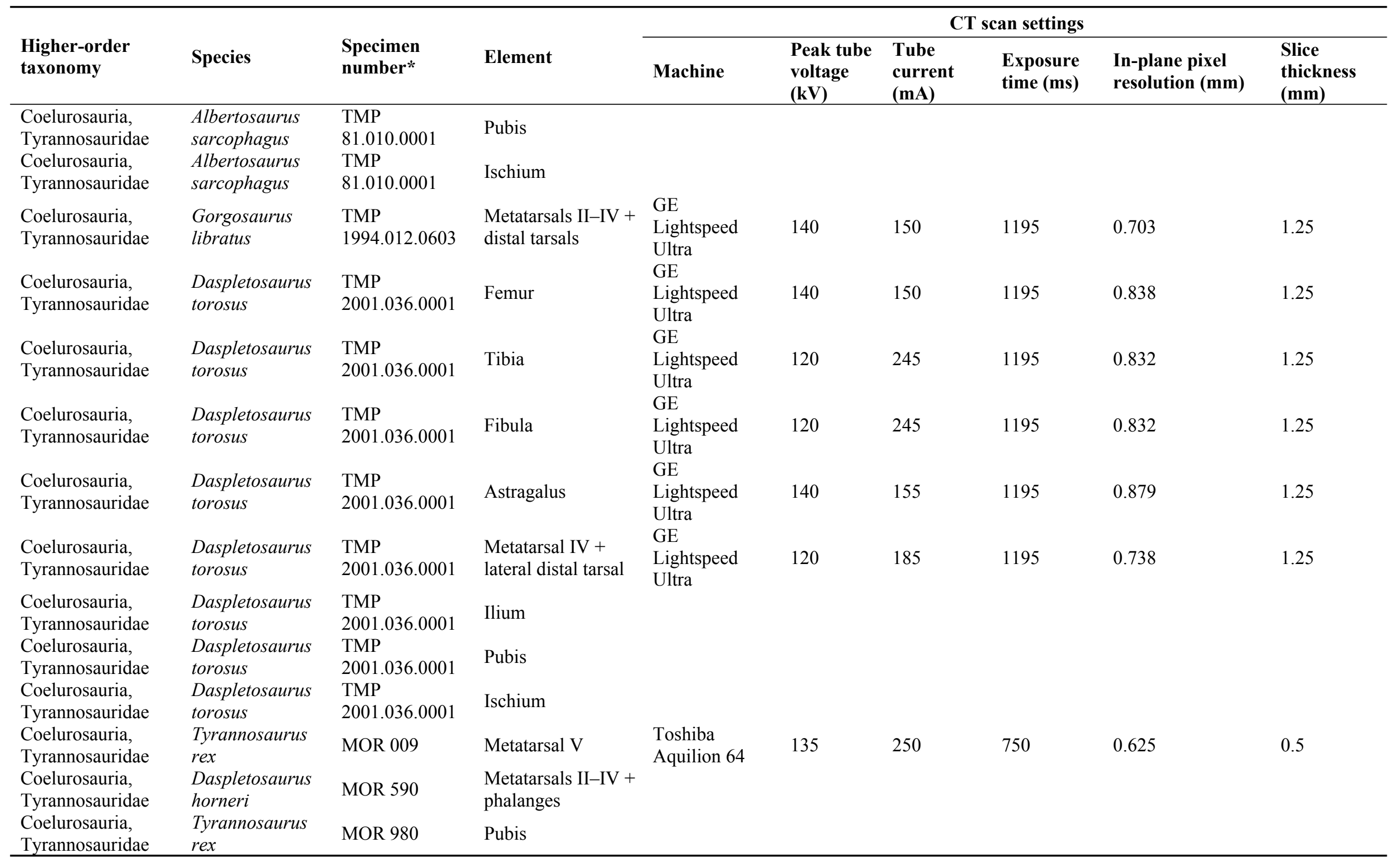


Table 1 (continued).

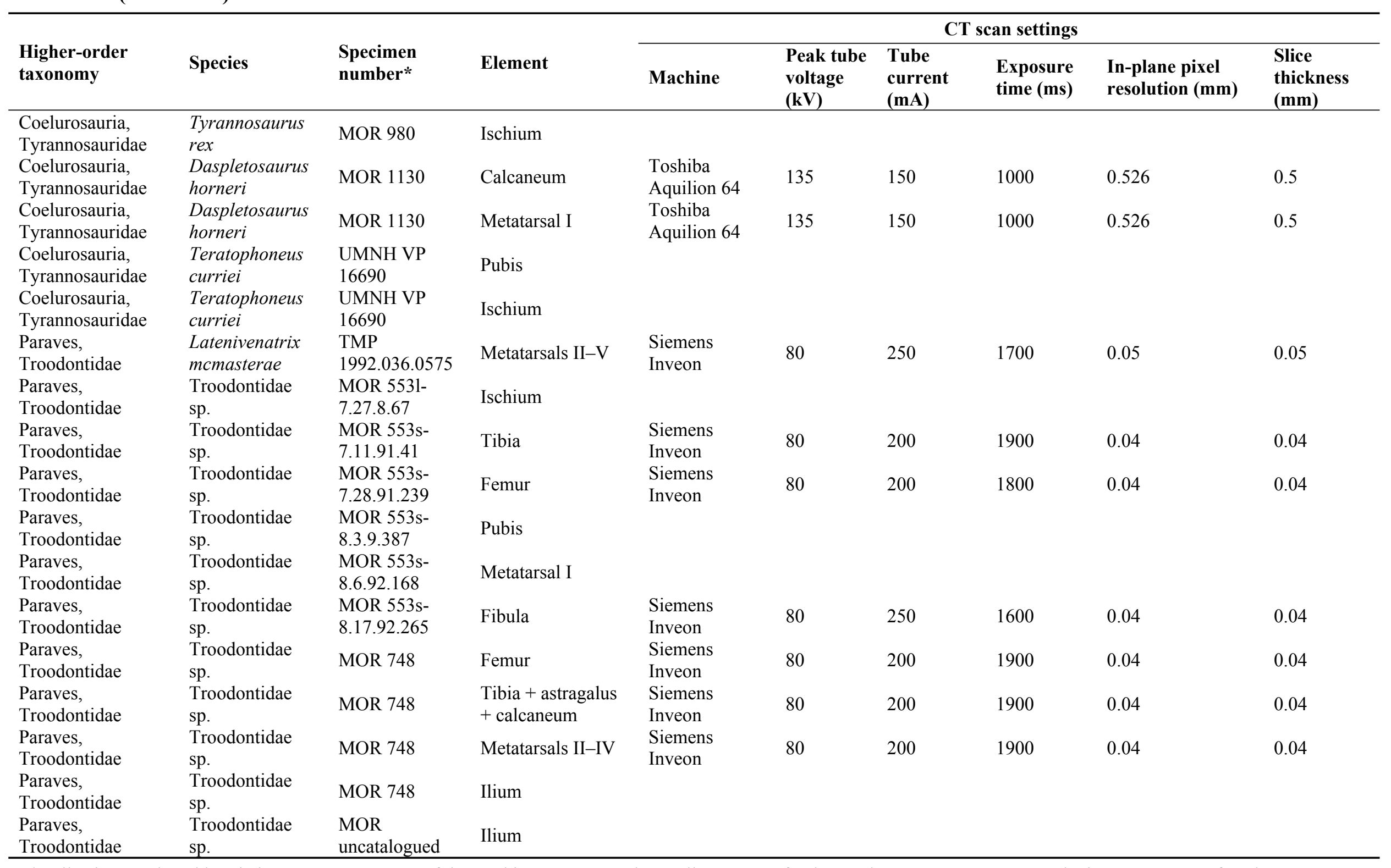

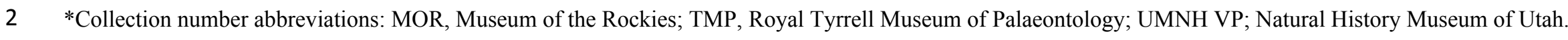




\section{Table 2 (on next page)}

The origins and insertions of each of the muscles and ligaments represented in the Daspletosaurus and' Troodon ' musculoskeletal models.

Specific differences between the two theropods are noted where appropriate. 
Table 2. The origins and insertions of each of the muscles and ligaments represented in the Daspletosaurus and 'Troodon' musculoskeletal models. Specific differences between the two theropods are noted where appropriate.

\begin{tabular}{|c|c|c|c|}
\hline Muscle or ligament & Abbreviation & Origin & Insertion \\
\hline Iliotibialis 1 & IT1 & Anterior rim of lateral ilium & Cnemial crest \\
\hline Iliotibialis 2 & IT2 & Dorsal rim of ilium, lateral surface & Cnemial crest \\
\hline Iliotibialis 3 & IT3 & Dorsal rim of postacetabular ilium & Cnemial crest \\
\hline Ambiens & AMB & Preacetabular process on proximal pubis & Cnemial crest \\
\hline Femorotibialis externus & FMTE & Lateral femoral shaft & Cnemial crest \\
\hline Femorotibialis internus & FMTI & Anteromedial femoral shaft & Cnemial crest \\
\hline Iliofibularis & ILFB & $\begin{array}{l}\text { Lateral postacetabular ilium, between IFE and FTE; posterior to } \\
\text { median vertical ridge of the ilium in Daspletosaurus }\end{array}$ & Fibular tubercle \\
\hline Iliofemoralis externus & IFE & $\begin{array}{l}\text { Lateral ilium, anterodosal to acetabulum; anterior to median } \\
\text { vertical ridge of the ilium in Daspletosaurus }\end{array}$ & Trochanteric shelf of femur \\
\hline $\begin{array}{l}\text { Iliotrochantericus } \\
\text { caudalis }\end{array}$ & ITC & Lateral preacetabular ilium & Lesser trochanter \\
\hline $\begin{array}{l}\text { Puboischiofemoralis } \\
\text { internus } 1\end{array}$ & PIFI1 & $\begin{array}{l}\text { Iliac preacetabular fossa; also descending onto lateral surface of } \\
\text { pubic peduncle in Daspletosaurus }\end{array}$ & Anteromedial aspect of proximal femur \\
\hline $\begin{array}{l}\text { Puboischiofemoralis } \\
\text { internus } 2\end{array}$ & PIFI2 & $\begin{array}{l}\text { Near PIFI1 origin, probably anterior to it (iliac preacetabular } \\
\text { fossa) }\end{array}$ & $\begin{array}{l}\text { Distal to lessor trochanter; on accessory trochanter in } \\
\text { Daspletosaurus }\end{array}$ \\
\hline $\begin{array}{l}\text { Flexor tibialis internus } \\
1\end{array}$ & FTI1 & $\begin{array}{l}\text { Low tubercle on posterolateral ischial shaft in Daspletosaurus; } \\
\text { distal end of ischium in 'Troodon' }\end{array}$ & Medial proximal tibia \\
\hline $\begin{array}{l}\text { Flexor tibialis internus } \\
3\end{array}$ & FTI3 & $\begin{array}{l}\text { Ischial tuberosity on posterolateral proximal ischium in } \\
\text { Daspletosaurus; proximal ischial shcaft in 'Troodon' }\end{array}$ & Medial proximal tibia \\
\hline Flexor tibialis externus & FTE & Lateral postacetabular ilium & Medial proximal tibia \\
\hline Adductor femoris 1 & ADD1 & Lateral surface of obturator process & $\begin{array}{l}\text { Medial posterodistal surface of femoral shaft; large scarred } \\
\text { region in Daspletosaurus }\end{array}$ \\
\hline Adductor femoris 2 & ADD2 & Posterodorsal rim of ischium & $\begin{array}{l}\text { Lateral posterodistal surface of femoral shaft; large scarred } \\
\text { region in Daspletosaurus }\end{array}$ \\
\hline $\begin{array}{l}\text { Puboischiofemoralis } \\
\text { externus } 1\end{array}$ & PIFE1 & Anterior surface of pubic apron & Greater trochanter \\
\hline $\begin{array}{l}\text { Puboischiofemoralis } \\
\text { externus } 2\end{array}$ & PIFE2 & Posterior surface of pubix apron & Greater trochanter \\
\hline $\begin{array}{l}\text { Puboischiofemoralis } \\
\text { externus } 3\end{array}$ & PIFE3 & Lateral ischium, between ADD1 and ADD2 & Greater trochanter \\
\hline Ischiotrochantericus & ISTR & Medial surface of ischium & Lateral proximal femur \\
\hline
\end{tabular}


Table 2 (continued).

\begin{tabular}{|c|c|c|c|}
\hline Muscle or ligament & Abbreviation & Origin & Insertion \\
\hline Caudofemoralis longus & CFL & $\begin{array}{l}\text { Caudal vertebral centra, probably from caudal vertebrae } 1-15 \text { in } \\
\text { Daspletosaurus and caudal vertebrae } 1-10 \text { in 'Troodon' }\end{array}$ & $\begin{array}{l}\text { Medial surface of fourth trochanter in Daspletosaurus, } \\
\text { posteromedial surface of proximal femur in 'Troodon' }\end{array}$ \\
\hline Caudofemoralis brevis & CFB & Brevis fossa of ilium & $\begin{array}{l}\text { Lateral surface of fourth trochanter in Daspletosaurus, } \\
\text { posterolateral surface of proximal femur in 'Troodon' }\end{array}$ \\
\hline Gastrocnemius lateralis & GL & Posterolateral surface of distal femur & Posterior surface of metatarsals II-IV \\
\hline Gastrocnemius medialis & GM & Medial proximal tibia & Posterior surface of metatarsals II-IV \\
\hline $\begin{array}{l}\text { Flexor digitorum } \\
\text { longus }\end{array}$ & FDL & Posterior surface of distal femur & Ventral aspect of digit II-IV phalanges \\
\hline Flexor digitorum brevis & FDB & Posterior surface of metatarsals II-IV & Ventral aspect of digit II-IV phalanges \\
\hline Flexor hallucis longus & FHL & Posterior surface of femur & Ventral aspect of digit I phalanges \\
\hline $\begin{array}{l}\text { Extensor digitorum } \\
\text { longus }\end{array}$ & EDL & $\begin{array}{l}\text { Distal anterolateral femur; possibly also proximal anterior tibia } \\
\text { in Daspletosaurus, and possibly also distal anterolateral femur } \\
\text { in 'Troodon' }\end{array}$ & Dorsal aspect of digit II-IV phalanges \\
\hline $\begin{array}{l}\text { Extensor digitorum } \\
\text { brevis }\end{array}$ & EDB & Anterior surface of metatarsals & Dorsal aspect of digit II-IV phalanges \\
\hline $\begin{array}{l}\text { Extensor hallucis } \\
\text { longus }\end{array}$ & EHL & Distal fibula & Dorsal aspect of digit I ungual \\
\hline Tibialis anterior & TA & Anterior surface of proximal tibia & Anteroproximal metatarsals II-IV \\
\hline Fibularis longus & FL & Anterolateral surface of tibia and/or fibula & Posterolateral ankle region (e.g., metatarsal V) \\
\hline Fibularis brevis & FB & Distal to FL on fibula & Anterolateral ankle region (e.g., metatarsal IV) \\
\hline $\begin{array}{l}\text { Knee medial collateral } \\
\text { ligament }\end{array}$ & KMCL & Depression on medial surface of medial femoral condyle & $\begin{array}{l}\text { Medial proximal tibiotarsus, proximal to FCLP and FCM } \\
\text { insertions }\end{array}$ \\
\hline $\begin{array}{l}\text { Knee lateral collateral } \\
\text { ligament }\end{array}$ & KLCL & Lateral surface of lateral femoral condyle & Lateral fibular head \\
\hline $\begin{array}{l}\text { Ankle medial collateral } \\
\text { ligament }\end{array}$ & AMCL & Depression on medial surface of astragalus & Medial proximal tarsometatarsus \\
\hline $\begin{array}{l}\text { Ankle lateral collateral } \\
\text { ligament }\end{array}$ & ALCL & Depression on lateral surface of calcaneum & Lateral proximal tarsometatarsus \\
\hline
\end{tabular}




\section{Table 3 (on next page)}

Hypothetical activities of the muscle actuators used in the Daspletosaurus and ' Troodon 'simulations.

$\mathrm{X}=$ active (capable of exerting up to two body weights of force), $\mathrm{O}=$ inactive (exerts zero force). 
1

2 Table 3. Hypothetical activities of the muscle actuators used in the Daspletosaurus and

3 'Troodon' simulations. $\mathrm{X}=$ active (capable of exerting up to two body weights of force), $\mathrm{O}$

4 = inactive (exerts zero force).

5

6

7

8

9

10

11

12

13

14

15

16

17

18

19

20

21

22

23

24

25

26

27

28

29

30

31

32

\begin{tabular}{ll}
\hline Muscle & Activity \\
\hline IT1 & $\mathrm{X}$ \\
IT2 & $\mathrm{X}$ \\
IT3 & $\mathrm{X}$ \\
AMB & $\mathrm{X}$ \\
FMTE & $\mathrm{X}$ \\
FMTI & $\mathrm{X}$ \\
ILFB & $\mathrm{X}$ \\
IFE & $\mathrm{X}$ \\
ITC & $\mathrm{X}$ \\
PIFI1 & $\mathrm{X}$ \\
PIFI2 & $\mathrm{X}$ \\
FTI1 & $\mathrm{X}$ \\
FTI3 & $\mathrm{X}$ \\
FTE & $\mathrm{X}$ \\
ADD1 & $\mathrm{X}$ \\
ADD2 & $\mathrm{X}$ \\
PIFE1 & $\mathrm{O}$ \\
PIFE2 & $\mathrm{O}$ \\
PIFE3 & $\mathrm{O}$ \\
ISTR & $\mathrm{X}$ \\
CFL & $\mathrm{X}$ \\
CFB & $\mathrm{X}$ \\
GL & $\mathrm{X}$ \\
GM & $\mathrm{X}$ \\
FDL & $\mathrm{X}$ \\
FDB & $\mathrm{X}$ \\
FHL & $\mathrm{X}$ \\
EDL & $\mathrm{O}$ \\
EDB & $\mathrm{O}$ \\
EHL & $\mathrm{O}$ \\
TA & $\mathrm{O}$ \\
FL & $\mathrm{O}$ \\
FB & $\mathrm{O}$ \\
\hline &
\end{tabular}




\section{Figure 1}

\section{The musculoskeletal model of the Daspletosaurus hindlimb developed in this study.}

This is shown in the 'neutral posture' for all joints, that is, when all joint angles are zero. (A-C) Geometries of the musculotendon actuators in relation to the bones, in lateral $(A)$, anterior $(B)$ and oblique anterolateral (C) views. (D-F) Location and orientation of joint coordinate systems (red, green and blue axes), the centres of mass for each segment (grey and white balls) and the soft tissue volumes used to calculate mass properties; these are shown in the same views as A-C. Also reported in D are the masses for each segment; the pelvis segment represents the body as well as the contralateral limb. In D-F, the flexion-extension axis of each joint is the blue axis. For scale, the length of each arrow in the triad of the global coordinate system is $500 \mathrm{~mm}$. 
A

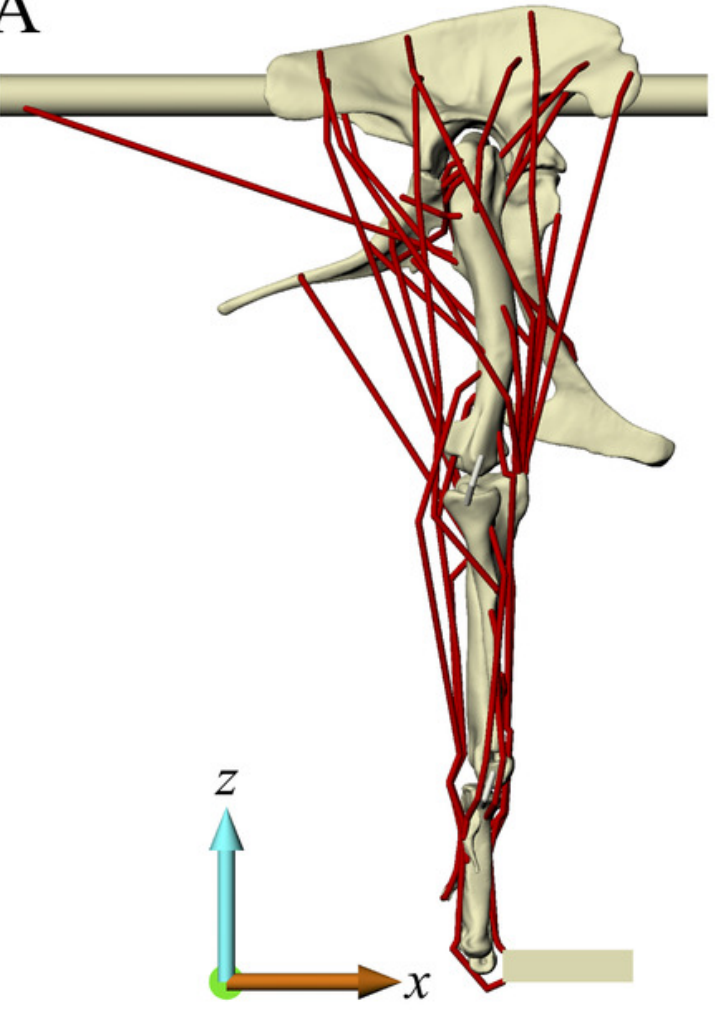

$\mathrm{D}$

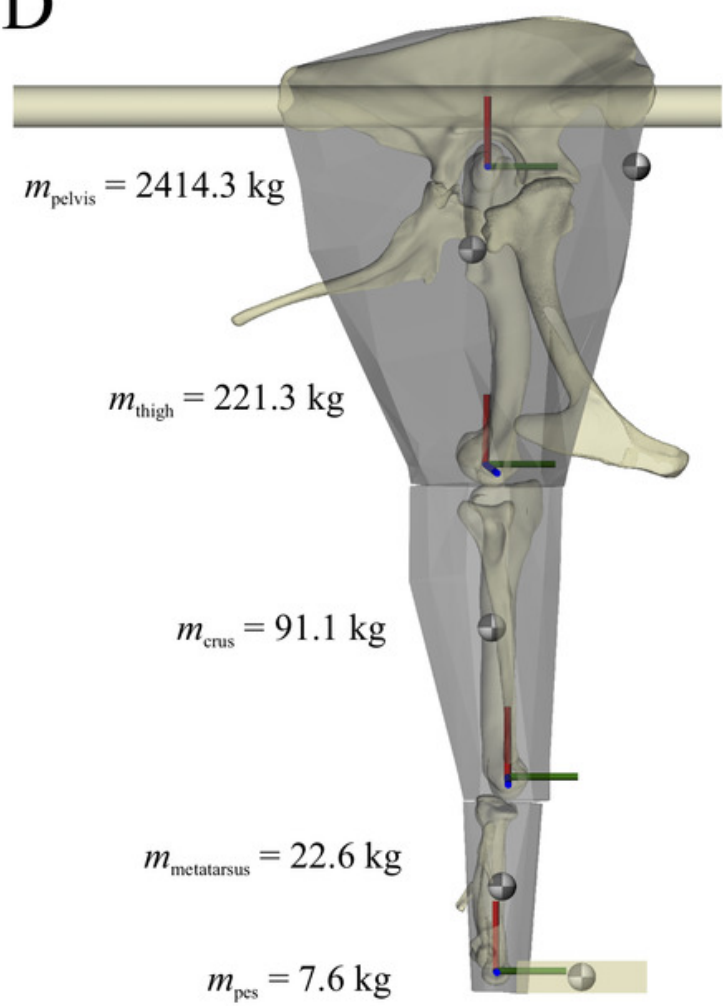

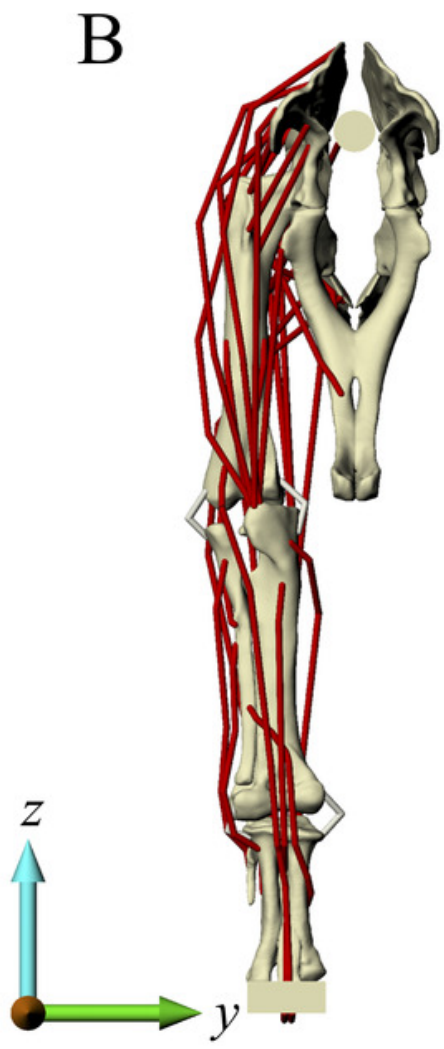

E

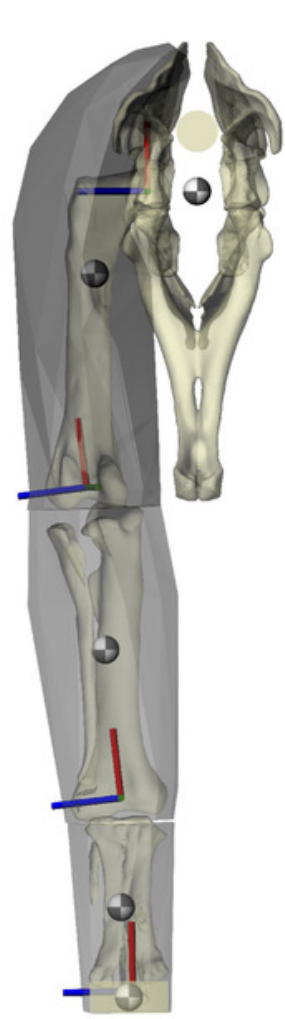

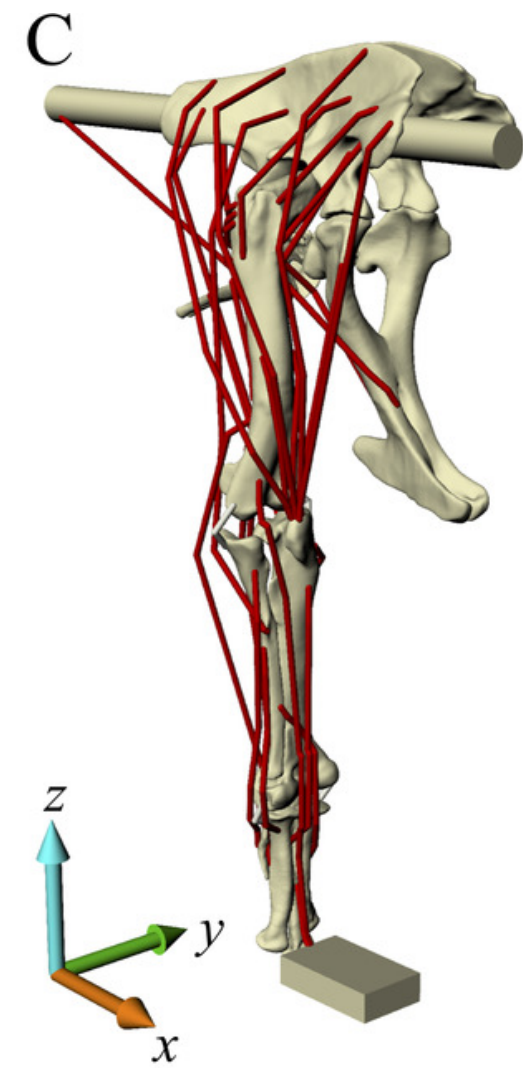

$\mathrm{F}$

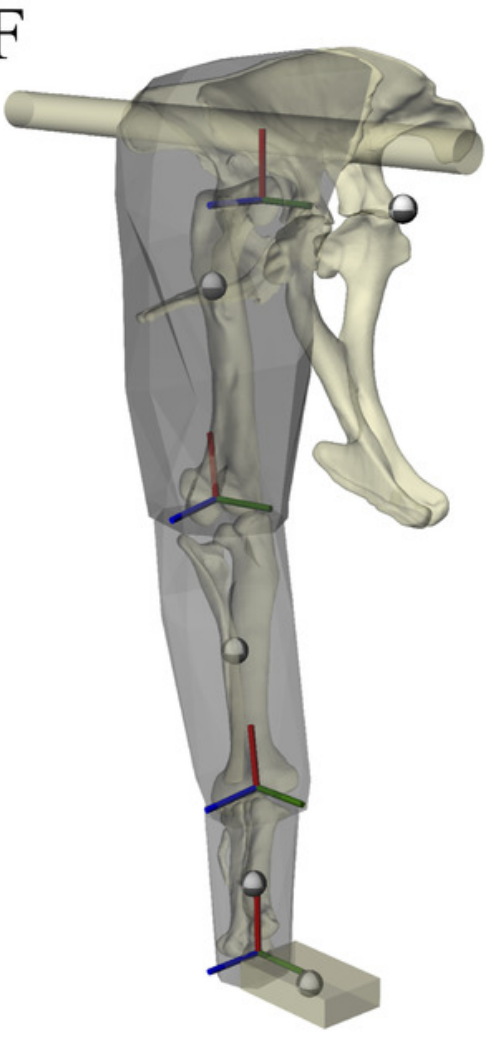




\section{Figure 2}

The musculoskeletal model of the 'Troodon 'hindlimb developed in this study.

This is shown in the neutral posture for all joints. A-C, geometries of the musculotendon actuators in relation to the bones, in lateral (A), anterior (B) and oblique anterolateral (C) views. (D-F) Location and orientation of joint coordinate systems (red, green and blue axes), the centres of mass for each segment (grey and white balls) and the soft tissue volumes used to calculate mass properties; these are shown in the same views as A-C. Also reported in $D$ are the masses for each segment; the pelvis segment represents the body as well as the contralateral limb. In D-F, the flexion-extension axis of each joint is the blue axis. For scale, the length of each arrow in the triad of the global coordinate system is $200 \mathrm{~mm}$. 
A

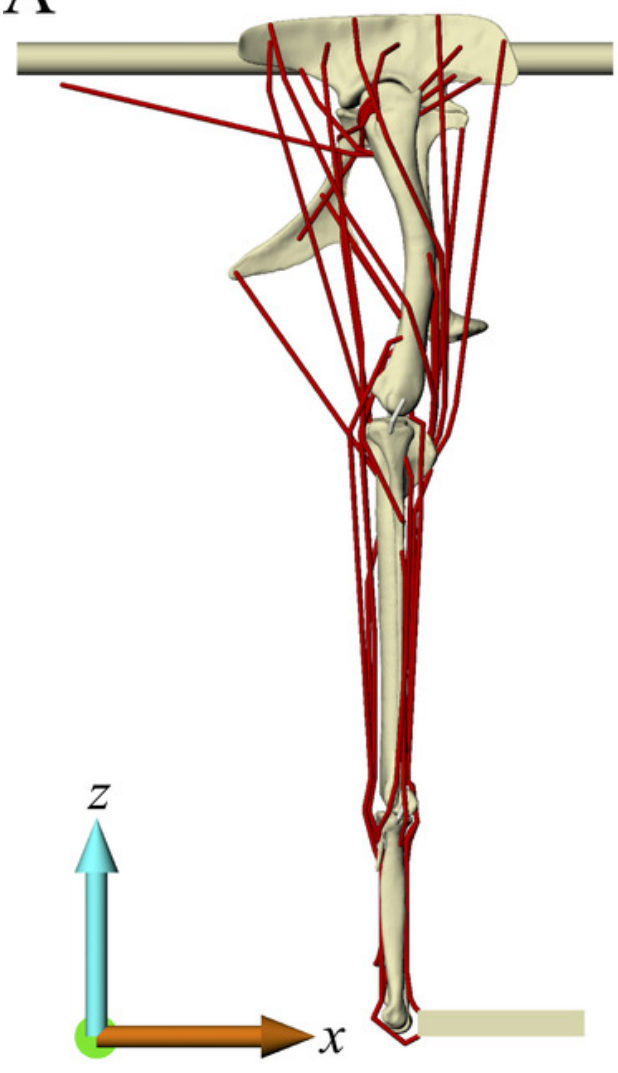

D

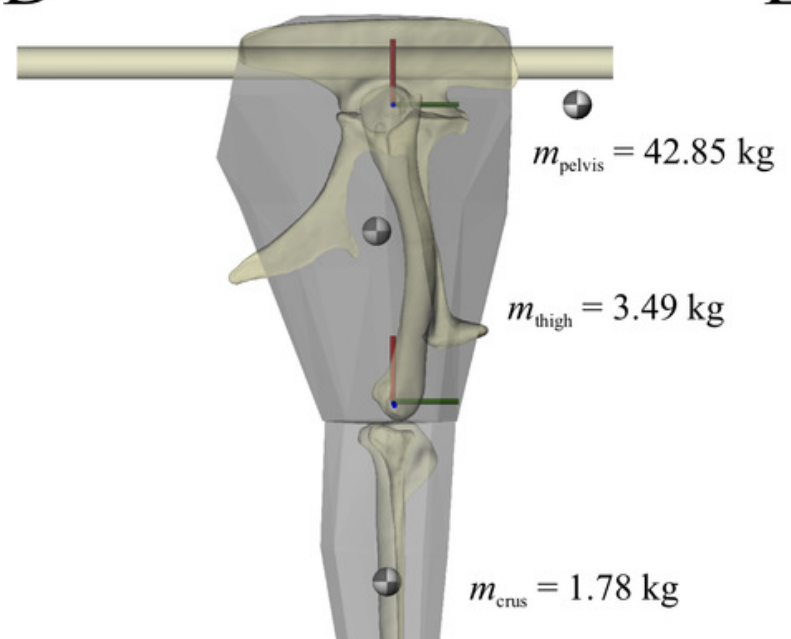

E

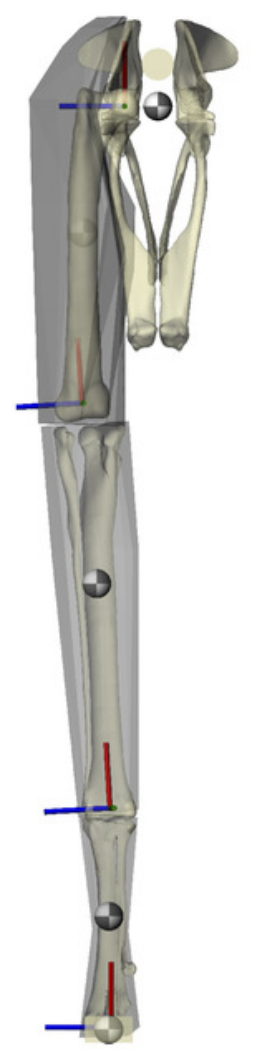

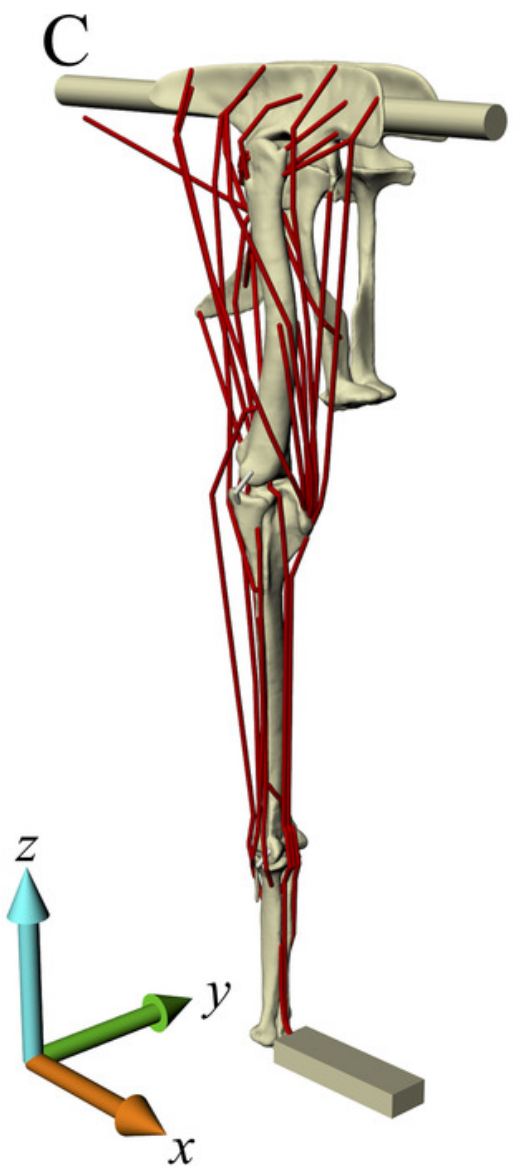

F

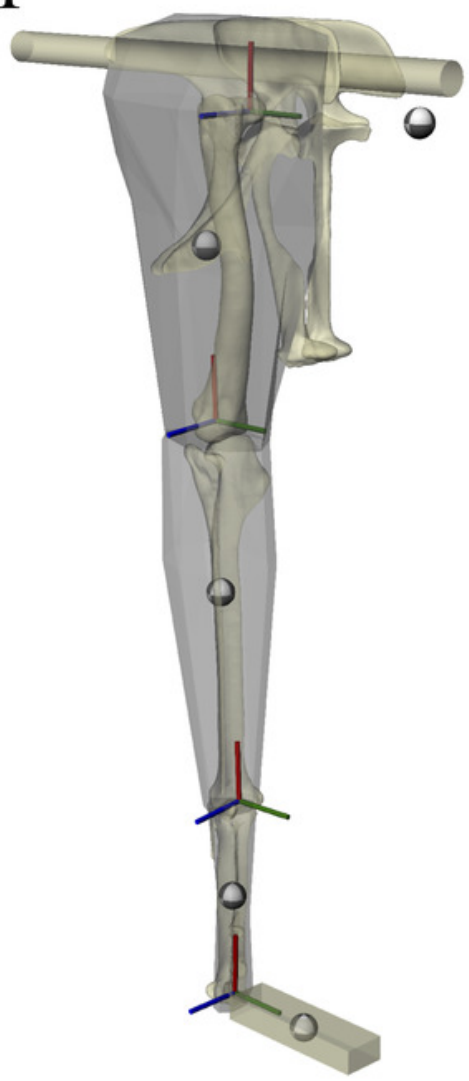




\section{Figure 3}

Varying the articulation of the hip joint in the Daspletosaurus model.

(A-C) The original 'solution posture' identified for the Daspletosaurus model. (D-F) The first variation in hip articulation, where the femur (and limb distal to it) is moved medially by 50 $\mathrm{mm}$. (G-I) The second variation in hip articulation, where the femur (and limb distal to it) is moved medially by $50 \mathrm{~mm}$, also with a sizeable amount of hip abduction and external longaxis rotation. A, D and $\mathrm{G}$ are in oblique anterolateral view; B, E and $\mathrm{H}$ are in close-ups of the hip articulation in anterior view; C, F and I show the whole hindlimb in anterior view, to illustrate the effect of differing hip articulations on gross limb position. Intervening soft tissues used in the finite element simulations are shown in turquoise; for clarity, the ilium and pubis are shown translucent in B, E and H. Also illustrated in B are the relative diameters of the femoral head (solid lines) and the acetabulum (dashed lines). 
A

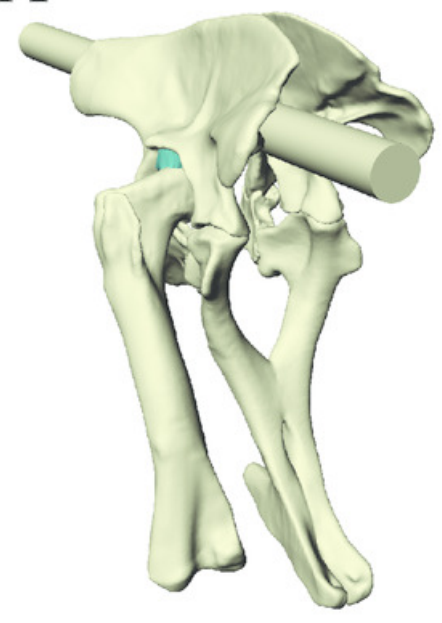

D

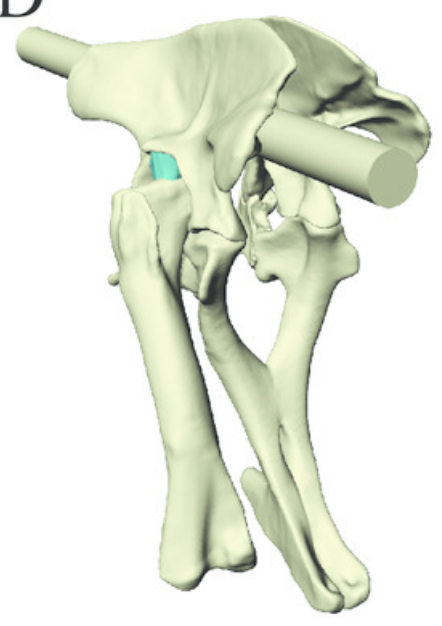

G

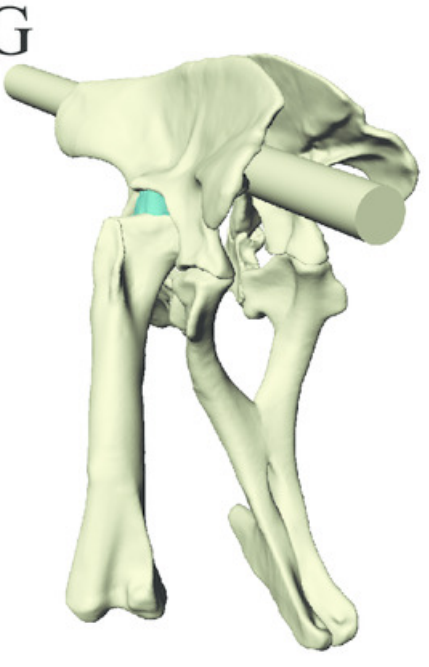

B

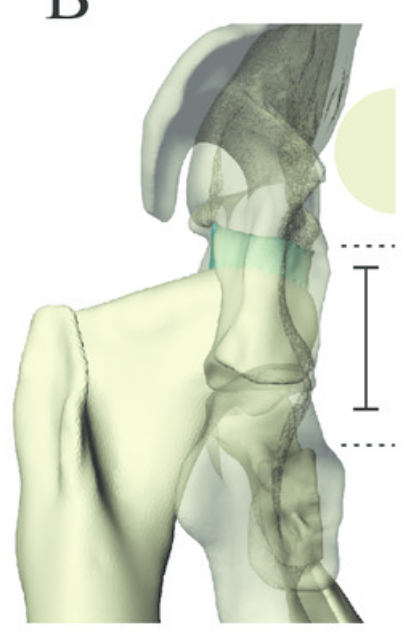

E

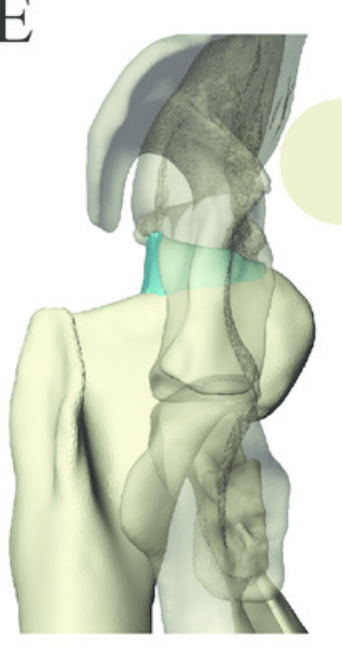

$\mathrm{H}$

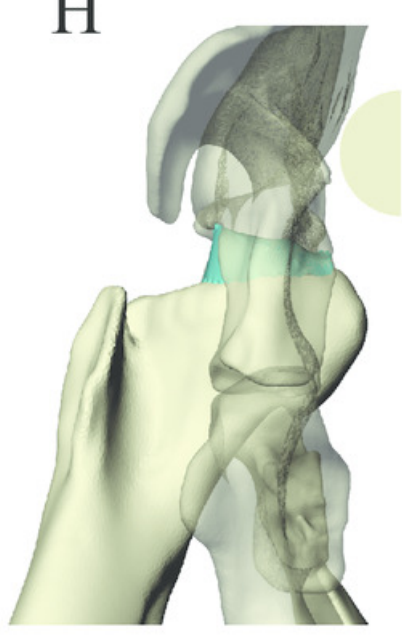

C

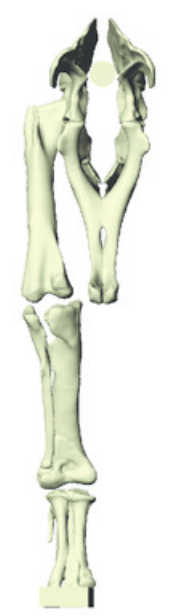

F

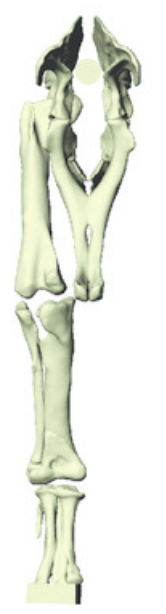

I

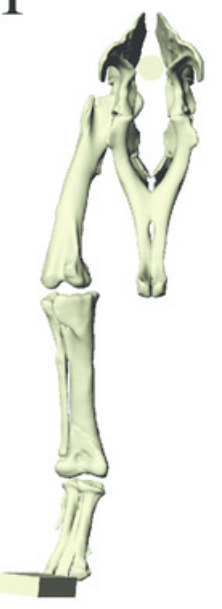




\section{Figure 4}

The postures tested for in Daspletosaurus .

Around the periphery are the different postures tested, shown in lateral view, with the final solution posture in the centre box, shown in lateral, dorsal and anterior views; the wholebody COM location is also shown for the solution posture in lateral view. Joint angles for each posture are given in blue font; hip joint angles are given in the order of flexion-extension, abduction-adduction and long-axis rotation. Hip extension angle is expressed relative to the horizontal, whereas knee and ankle angles are expressed relative to the femur and tibiotarsus (respectively). For the other hip angles, positive values indicate abduction and external rotation, whereas negative values indicate adduction and internal rotation. The metatarsophalangeal joint angle is expressed relative to the neutral posture. The angular deviation between $\boldsymbol{\sigma}_{3}$ and $\mathbf{u}_{1}$ for each posture is also given in red font (reported as femoral head, then medial femoral condyle). The solution posture resulted in the greatest degree of overall correspondence between principal stress trajectories and observed cancellous bone architectural patterns in birds, as assessed by qualitative comparisons across the femur, tibiotarsus and fibula, as well as quantitative results for the femoral head and medial femoral condyle. 


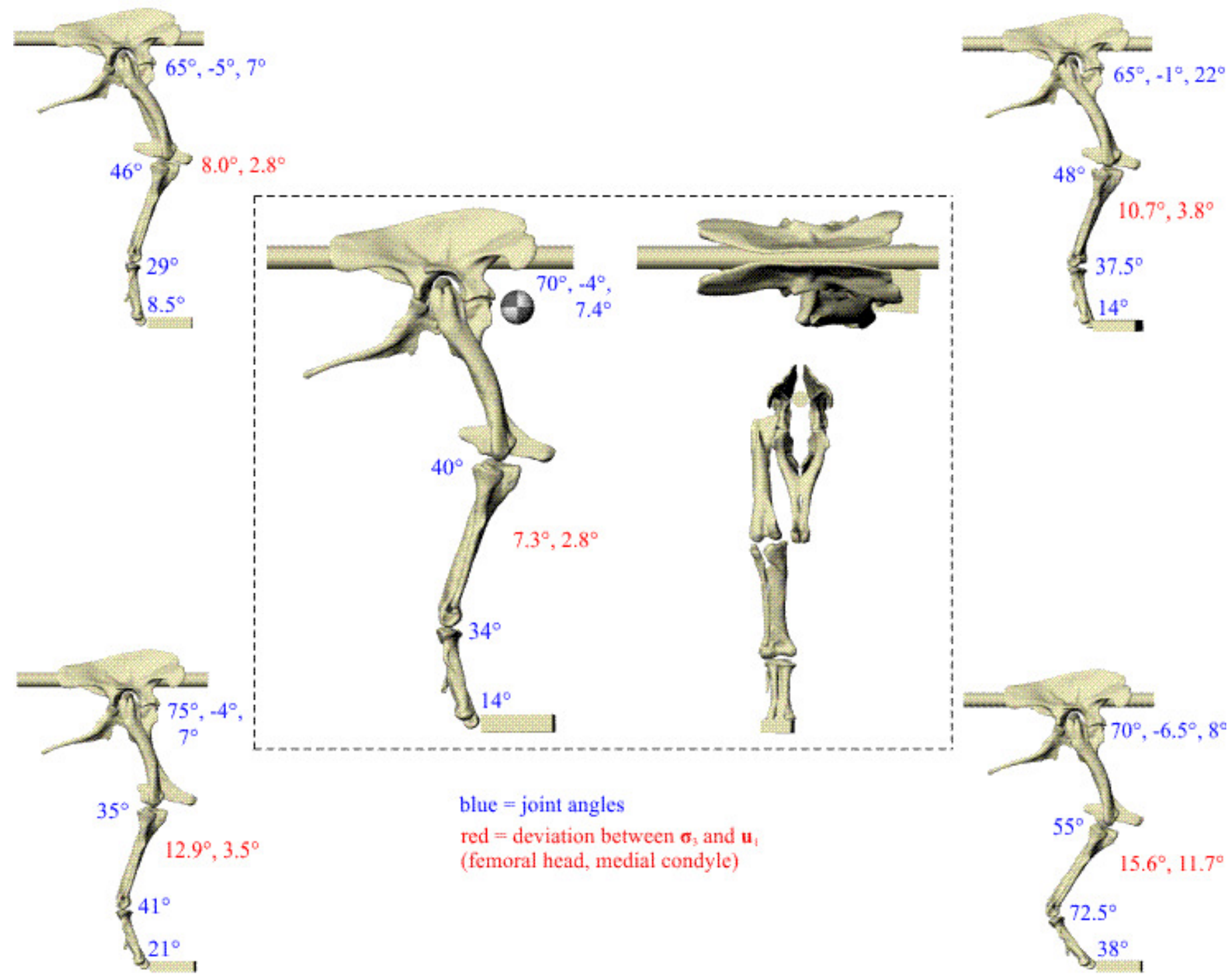




\section{Figure 5}

The postures tested for in 'Troodon'.

Around the periphery are the different postures tested, shown in lateral view, with the final solution posture in the centre box, shown in lateral, dorsal and anterior views; the wholebody COM location is also shown for the solution posture in lateral view. Joint angles for each posture are given in blue font, following the same conventions as Fig. 4. The angular deviation between $\boldsymbol{\sigma}_{3}$ and $\mathbf{u}_{1}$ for each posture is also given in red font (reported as femoral head, then medial femoral condyle). The solution posture resulted in the greatest degree of overall correspondence between principal stress trajectories and observed cancellous bone architectural patterns in birds, as assessed by qualitative comparisons across the femur, tibiotarsus and fibula, as well as quantitative results for the femoral head and medial femoral condyle. 


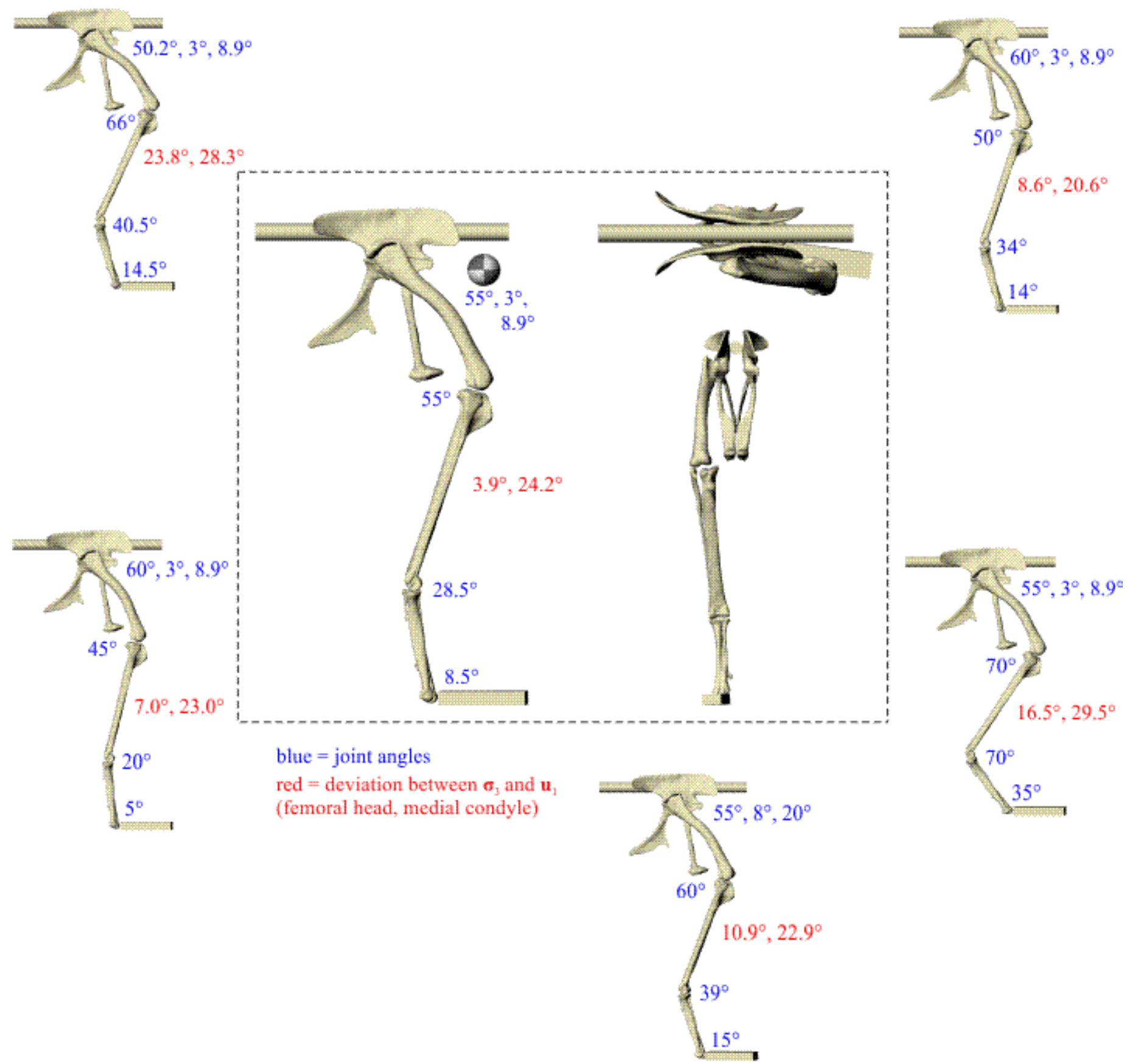




\section{Figure 6}

Principal stress trajectories for the proximal femur in the solution posture of Daspletosaurus, compared with observed cancellous bone fabric.

For easier visual comparison, the stress trajectories were 'downsampled' in a custom MATLAB script, by interpolating the raw stress results at each finite element node to a regular grid. (A) Vector field of $\boldsymbol{\sigma}_{1}$ (red) and $\boldsymbol{\sigma}_{3}$ (blue) in a 3-D slice through the proximal femur, parallel to the coronal plane and through the middle of the femoral head, in anterior view. Note how the trajectory of $\boldsymbol{\sigma}_{3}$ projects towards the apex of the femoral head (green braces). ( B) Observed cancellous bone architecture in the proximal femur of Allosaurus and tyrannosaurids (cf. Part I), in the same view as A. (C) Vector field of $\boldsymbol{\sigma}_{1}$ and $\boldsymbol{\sigma}_{3}$ in a 3-D slice through the lesser trochanter, parallel to the plane of the trochanter, in anterolateral view. (D) Observed cancellous bone architecture in the lesser trochanter of Allosaurus and tyrannosaurids (cf. Part I), in the same view as C. (E) Vector field of $\boldsymbol{\sigma}_{3}$ in the femoral head, shown as a 3-D slice parallel to the sagittal plane and through the apex of the head, in medial view. (F) Observed cancellous bone architecture in the femoral head of Allosaurus and tyrannosaurids (cf. Part I), in the same view as E. ( G) C omparison of the mean direction of $\boldsymbol{\sigma}_{3}$ in the femoral head (blue) and the estimated mean direction of $\mathbf{u}_{1}$ for Allosaurus and tyrannosaurids (red), plotted on an equal-angle stereoplot with northern hemisphere projection (using StereoNet 9.5; Allmendinger et al. 2013; Cardozo \& Allmendinger 2013) . Inset shows location of region for which the mean direction of $\boldsymbol{\sigma}_{3}$ was calculated. 

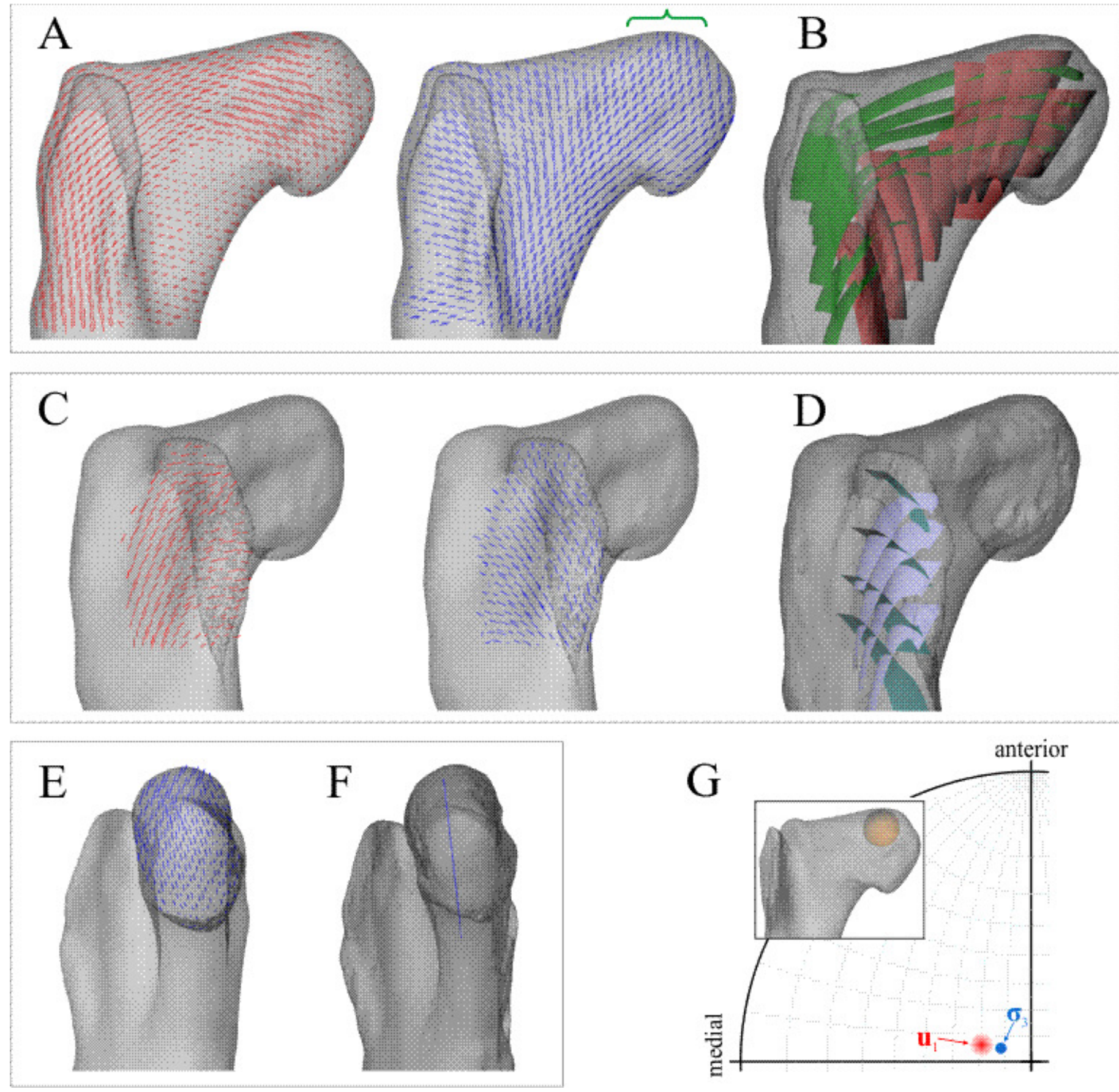


\section{Figure 7}

Principal stress trajectories for the distal femur and fourth trochanter in the solution posture of Daspletosaurus ,compared with observed cancellous bone fabric.

(A) $V$ ector field of $\boldsymbol{\sigma}_{1}$ (red) and $\boldsymbol{\sigma}_{3}$ (blue) in a 3-D slice, parallel to the coronal plane and through the anterior aspect of the distal metaphysis, in anterior view. (B ) Observed cancellous bone architecture in the distal metaphysis of Allosaurus and tyrannosaurids (cf. Part I), in the same view as A. ( C) Vector field of $\boldsymbol{\sigma}_{1}$ in the fourth trochanter, in medial view. (D) O bserved cancellous bone architecture in the fourth trochanter of Allosaurus and tyrannosaurids (cf. Part I), in the same view as C. ( E) Vector field of $\boldsymbol{\sigma}_{3}$ in the lateral condyle, shown as a 3-D slice parallel to the sagittal plane and through the middle of the condyle. (F) O bserved cancellous bone architecture in the lateral condyle of Allosaurus and tyrannosaurids (cf. Part I), in the same view as E. ( G) Vector field of $\boldsymbol{\sigma}_{3}$ in the medial condyle, shown as a 3-D slice parallel to the sagittal plane and through the middle of the condyle. $(\mathrm{H}) \mathrm{O}$ bserved cancellous bone architecture in the medial condyle of Allosaurus and tyrannosaurids (cf. Part I), in the same view as G. ( I) C omparison of the mean direction of $\boldsymbol{\sigma}$ ${ }_{3}$ in the medial condyle (blue) and the estimated mean direction of $\mathbf{u}_{1}$ for Allosaurus and tyrannosaurids (red), plotted on an equal-angle stereoplot with southern hemisphere projection. Inset shows location of region for which the mean direction of $\boldsymbol{\sigma}_{3}$ was calculated. 

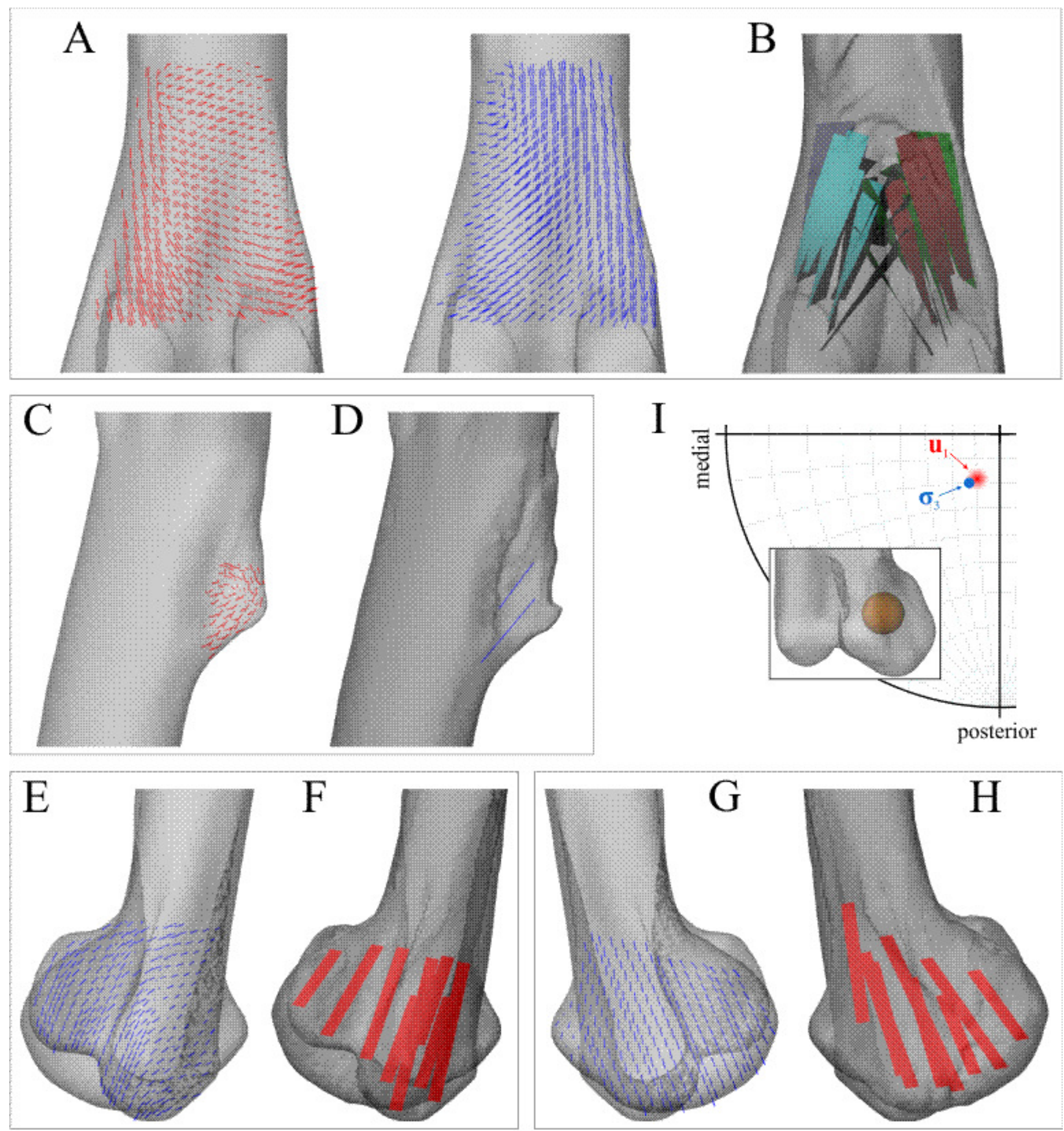


\section{Figure 8}

Principal stress trajectories for the tibia and fibula in the solution posture for Daspletosaurus, compared with observed cancellous bone fabric.

(A) Vector field of $\boldsymbol{\sigma}_{3}$ in the medial tibial condyle, shown as a 3-D slice through the middle of the condyle and parallel to the sagittal plane, in medial view. (B) O bserved cancellous bone architecture in the medial tibial condyle of Allosaurus and tyrannosaurids (cf. Part I), in the same view as A. ( C) Vector field of $\boldsymbol{\sigma}_{3}$ in the medial and lateral tibial condyles, shown as 3-D slices through the middle of the condyles and parallel to the coronal plane, in posterior view. (D) O bserved cancellous bone architecture in the medial and lateral tibial condyles of Allosaurus and tyrannosaurids (cf. Part I), in the same view as C. ( E) Vector field of $\boldsymbol{\sigma}_{3}$ in the lateral tibial condyle, shown as a 3-D slice through the middle of the condyle and parallel to the sagittal plane, in lateral view. (F) Observed cancellous bone architecture in the lateral tibial condyle of Allosaurus and tyrannosaurids (cf. Part I), in the same view as E. ( G) Vector field of $\boldsymbol{\sigma}_{1}$ in the cnemial crest, shown as a 3-D slice parallel to the coronal plane, in anterior view. $(\mathrm{H})$ Observed cancellous bone architecture in cnemial crest of Allosaurus and tyrannosaurids (cf. Part I), sectioned in the plane of the crest, shown in the same view as G; blue section lines illustrate primary architectural direction. ( I) Vector field of $\boldsymbol{\sigma}_{1}$ in the cnemial crest, shown as a 3-D slice parallel to the sagittal plane, in medial view. ( J) Observed cancellous bone architecture in cnemial crest of Allosaurus and tyrannosaurids (cf. Part I), sectioned in the plane of the crest, shown in the same view as I. ( K) Vector field of $\boldsymbol{\sigma}_{3}$ in the medial aspect of the fibular head, in medial view. ( L) Observed cancellous bone architecture in the fibular head of Allosaurus and tyrannosaurids (cf. Part I), in the same view as $\mathrm{K}$. 

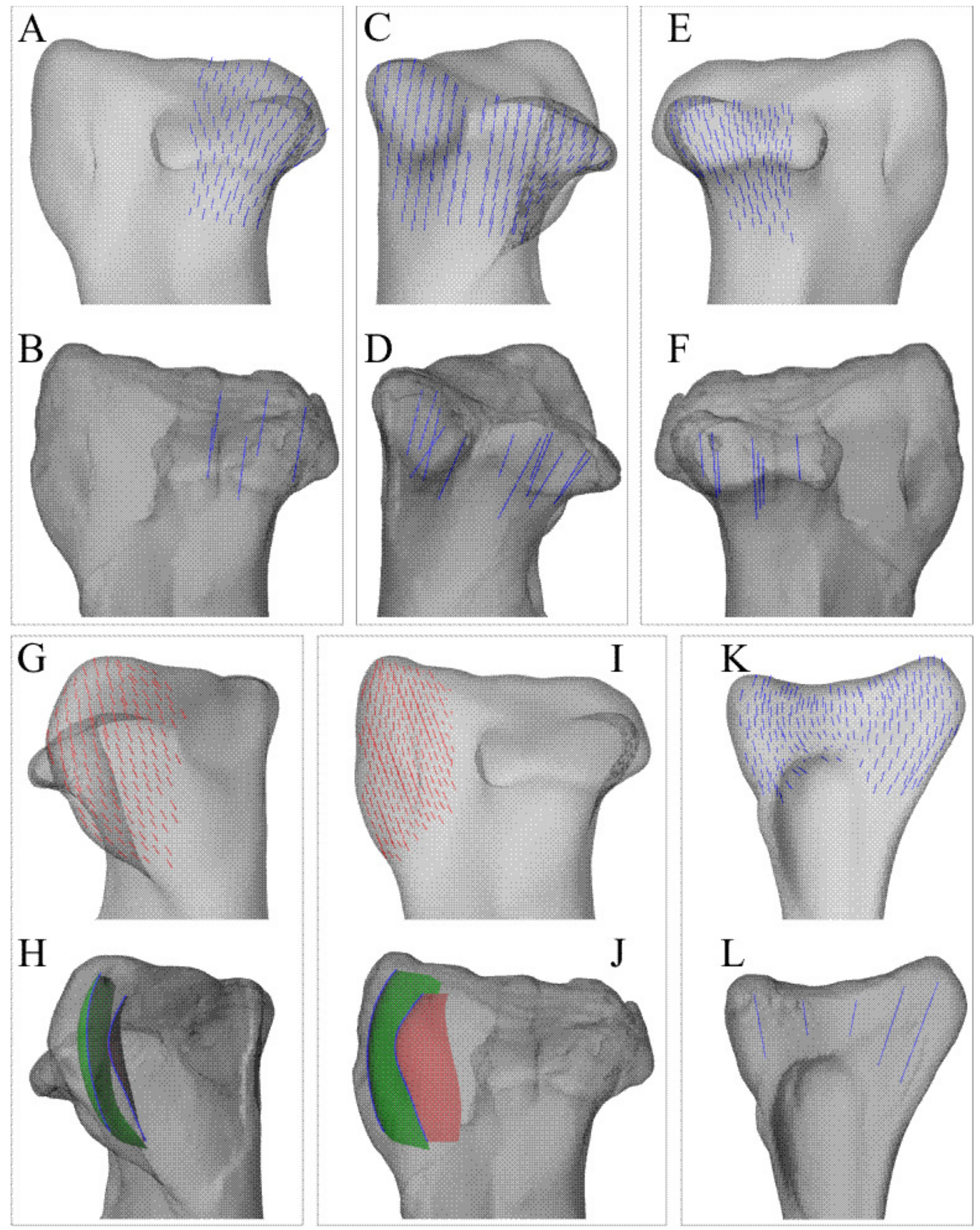


\section{Figure 9}

Principal stress trajectories for the proximal femur in the solution posture of 'Troodon ', compared with observed cancellous bone fabric.

(A, B) Vector field of $\boldsymbol{\sigma}_{3}$ in the femoral head, shown as 3-D slices parallel to the coronal plane ( $A$, in anterior view) and sagittal plane (B, in medial view). (C, D) Observed vector field of $\mathbf{u}_{1}$ in the femoral head, in the same views as A and B, respectively (cf. Part I) . (E) C omparison of the mean direction of $\boldsymbol{\sigma}_{3}$ in the femoral head (blue) and the mean direction of $\mathbf{u}_{1}$ (red), plotted on an equal-angle stereoplot with northern hemisphere projection. Inset shows location of region for which the mean direction of $\boldsymbol{\sigma}_{3}$ was calculated. $(F, G)$ Vector field of $\boldsymbol{\sigma}_{3}$ under the greater trochanter, shown as 3-D slices parallel to the coronal plane ( $F$, in posterior view) and sagittal plane ( $G$, in lateral view). $(H, I)$ Observed vector field of $\mathbf{u}_{1}$ under the greater trochanter, shown in the same views as F and G, respectively (cf. Part I) . (J) Vector field of $\boldsymbol{\sigma}_{1}$ in the lesser trochanter, shown in oblique anterolateral view. (K) Observed vector field of $\mathbf{u}_{1}$ in the lesser trochanter, shown in the same view as $\mathrm{J}$ for both specimens studied (cf. Part I) . 

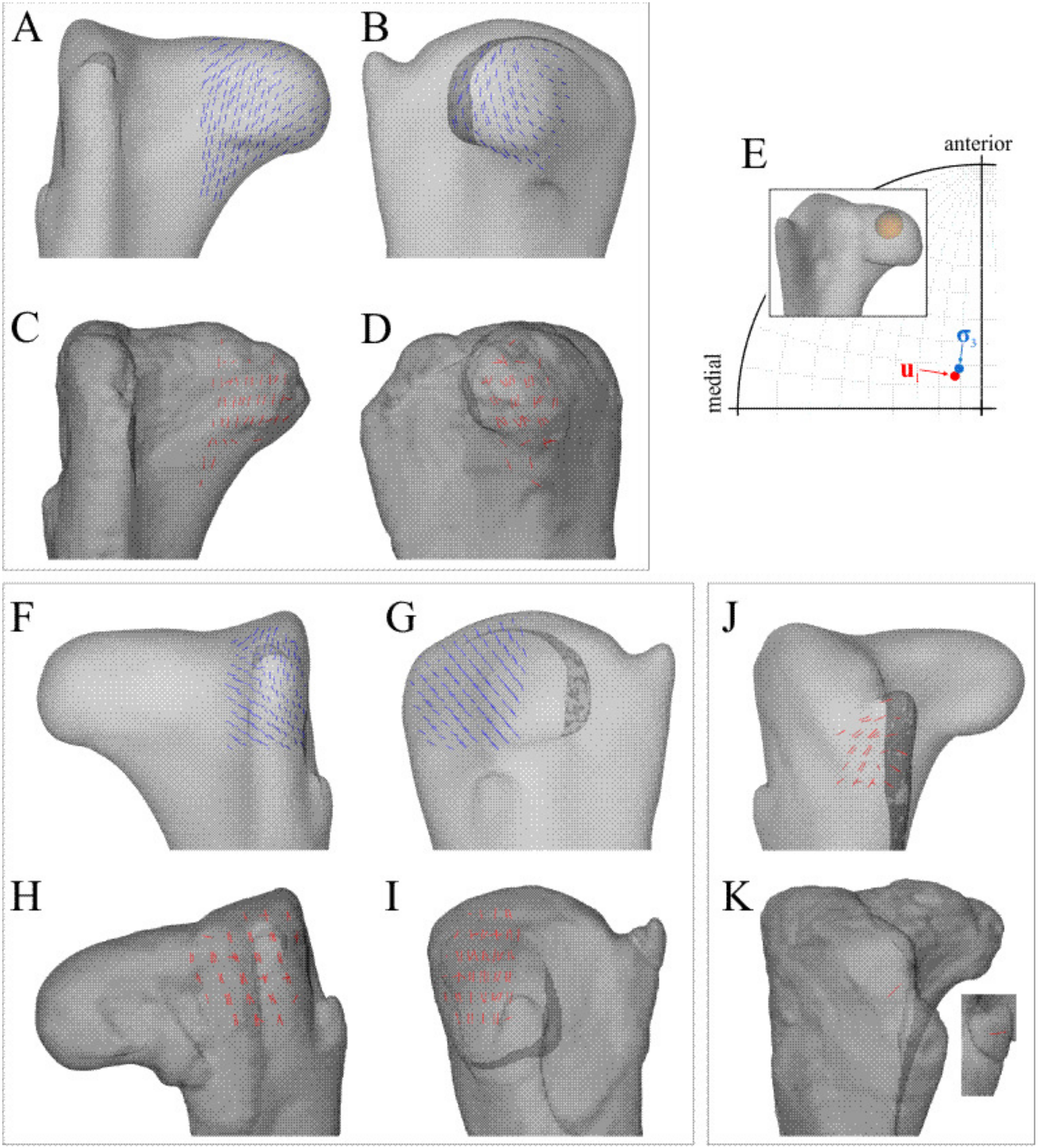


\section{Figure 10}

Principal stress trajectories for the distal femoral condyles in the solution posture of 'Troodon', compared with observed cancellous bone fabric.

(A) Vector field of $\boldsymbol{\sigma}_{3}$ in the lateral condyle, shown as a 3-D slice parallel to the sagittal plane. (B) Observed vector field of $\mathbf{u}_{1}$ in the lateral condyle, shown in the same view as $A$ (cf. Part I) . (C) Vector field of $\boldsymbol{\sigma}_{3}$ in the medial condyle, shown as a 3-D slice parallel to the sagittal plane. (D) Observed vector field of $\mathbf{u}_{1}$ in the medial condyle, shown in the same view as C (cf. Part I) . (E) C omparison of the mean direction of $\boldsymbol{\sigma}_{3}$ in the medial condyle (blue) and the mean direction of $\mathbf{u}_{1}$ (red), plotted on an equal-angle stereoplot with southern hemisphere projection. This shows that in the solution posture the mean direction of $\boldsymbol{\sigma}_{3}$ was of the same general azimuth as the mean direction of $\mathbf{u}_{1}$, but was markedly more posteriorly inclined. Inset shows location of region for which the mean direction of $\boldsymbol{\sigma}_{3}$ was calculated.
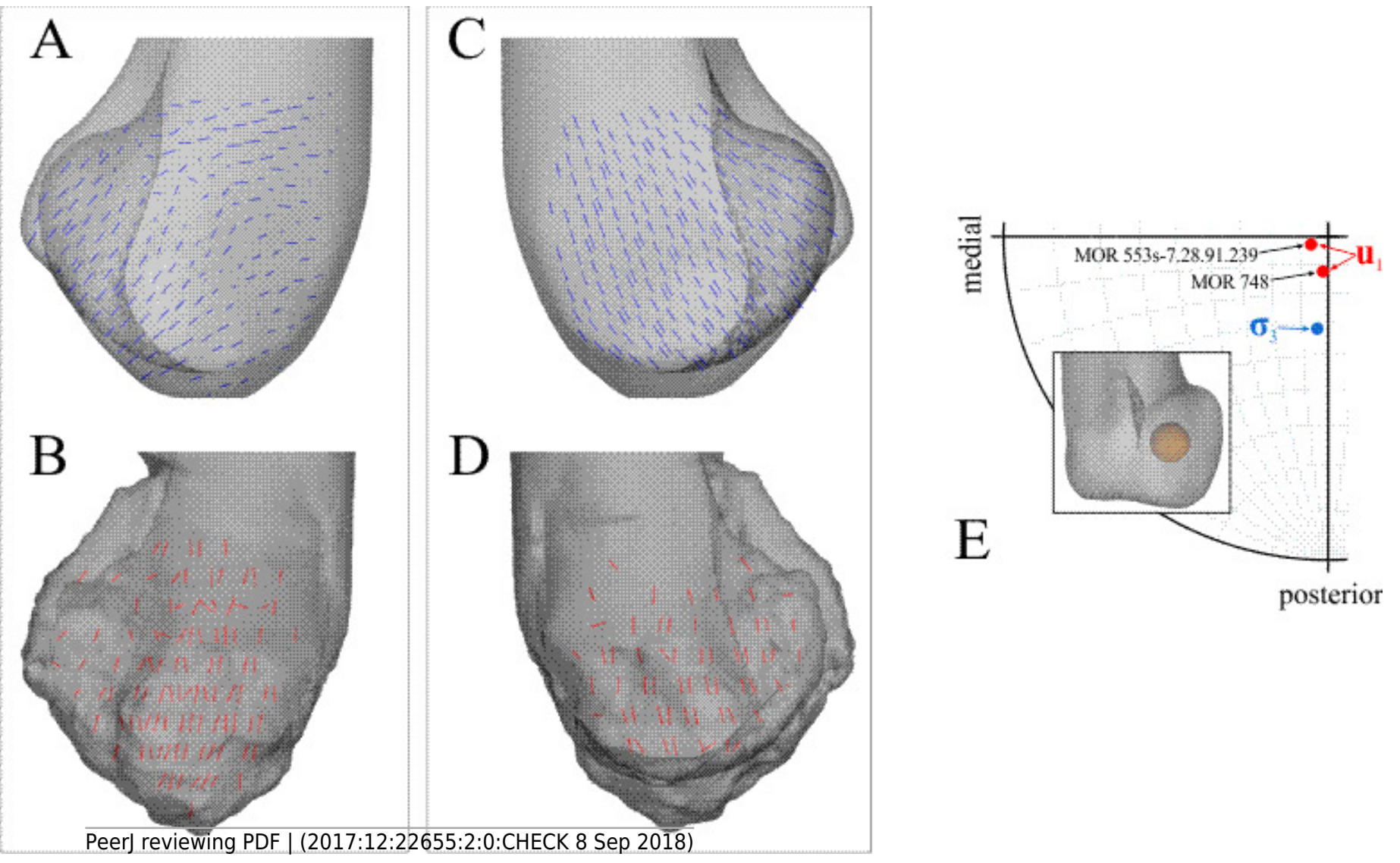


\section{Figure 11}

Principal stress trajectories for the tibia and fibula in the solution posture for 'Troodon', compared with observed cancellous bone fabric.

(A) Vector field of $\boldsymbol{\sigma}_{3}$ in the medial tibial condyle, shown as a 3-D slice through the middle of the condyle and parallel to the sagittal plane, in medial view. (B) Observed vector field of $\mathbf{u}_{1}$ in the medial tibial condyle, in the same view as A (cf. Part I) . (C) Vector field of $\boldsymbol{\sigma}_{3}$ in the medial and lateral tibial condyles, shown as 3-D slices through the middle of the condyles and parallel to the coronal plane, in posterior view. (D) Observed vector field of $\mathbf{u}_{1}$ in the medial and lateral tibial condyles, in the same view as C (cf. Part I) . (E) Vector field of $\boldsymbol{\sigma}_{3}$ in the lateral tibial condyle, shown as a 3-D slice through the middle of the condyle and parallel to the sagittal plane, in lateral view. (F) Observed vector field of $\mathbf{u}_{1}$ in the lateral tibial condyle, in the same view as E (cf. Part I) . (G) Vector field of $\boldsymbol{\sigma}_{1}$ in the cnemial crest, shown as a 3-D slice parallel to the coronal plane, in anterior view. (H) Observed vector field of $\mathbf{u}_{1}$ in the cnemial crest, in the same view as G (cf. Part I) . (I) Vector field of $\boldsymbol{\sigma}_{1}$ in the cnemial crest, shown as a 3-D slice parallel to the sagittal plane, in medial view. (J) Observed vector field of $\mathbf{u}_{1}$ in the cnemial crest, in the same view as I (cf. Part I) . (K) Vector field of $\boldsymbol{\sigma}_{1}$ in the lateral fibular head, in lateral view. (L) Vector field of $\boldsymbol{\sigma}_{3}$ in the medial fibular head, in medial view (reversed). ( M) Observed vector field of $\mathbf{u}_{1}$ in the fibular head, in the same view as $\mathrm{K}$ (cf. Part I) . 

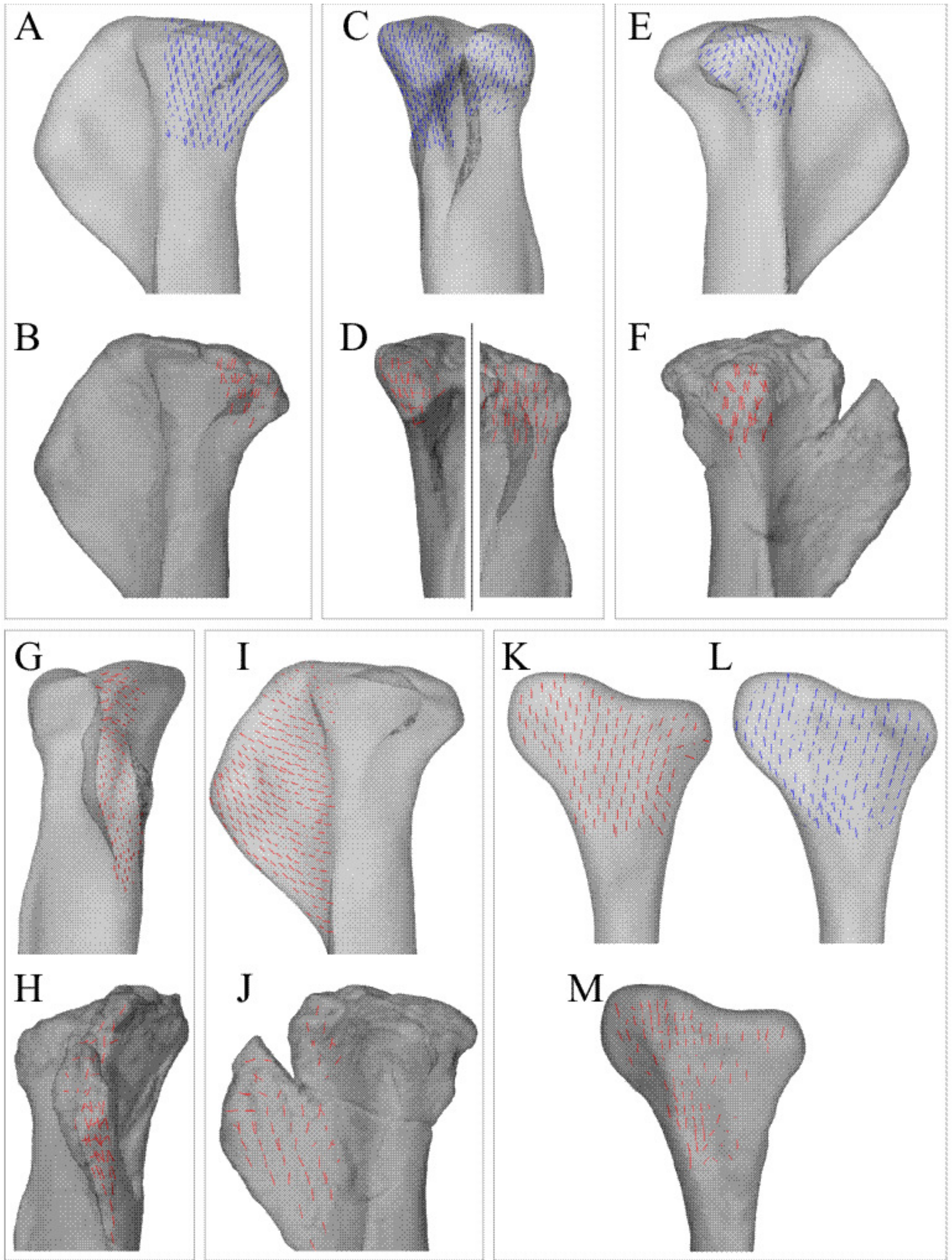

PeerJ reviewing PDF | (2017:12:22655:2:0:CHECK 8 Sep 2018) 


\section{Figure 12}

Principal stress trajectories for the proximal femur of Daspletosaurus in the two variations in hip articulation tested.

(A) $V$ ector field of $\boldsymbol{\sigma}_{3}$ in the first variation tested, shown as a 3-D slice parallel to the coronal plane and through the middle of the femoral head. ( B) $V$ ector field of $\boldsymbol{\sigma}_{3}$ in the first variation tested, shown as a 3-D slice parallel to the sagittal plane and through the apex of the femoral head. (C) $\vee$ ector field of $\boldsymbol{\sigma}_{3}$ in the second variation tested, shown as a 3-D slice parallel to the coronal plane and through the middle of the femoral head. ( D) V ector field of $\boldsymbol{\sigma}_{3}$ in the second variation tested, shown as a 3-D slice parallel to the sagittal plane and through the apex of the femoral head. $A$ and $C$ are in anterior view, $B$ and $D$ are in medial view. Note in particular how the trajectory of $\boldsymbol{\sigma}_{3}$ projects towards the more cylindrical part of the femoral head, lateral to the apex (green braces); compare to Fig. $6 \mathrm{~A}, \mathrm{~B}, \mathrm{E}, \mathrm{F}$. Also note in $\mathrm{C}$ how $\boldsymbol{\sigma}_{3}$ has a strong medial component near the apex of the head. 
A

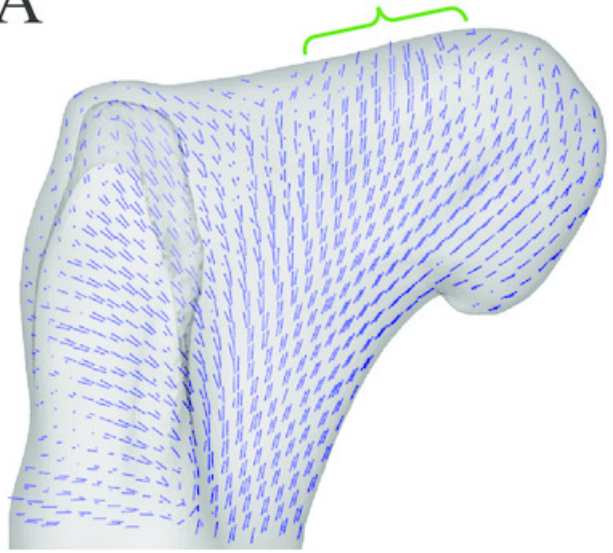

C

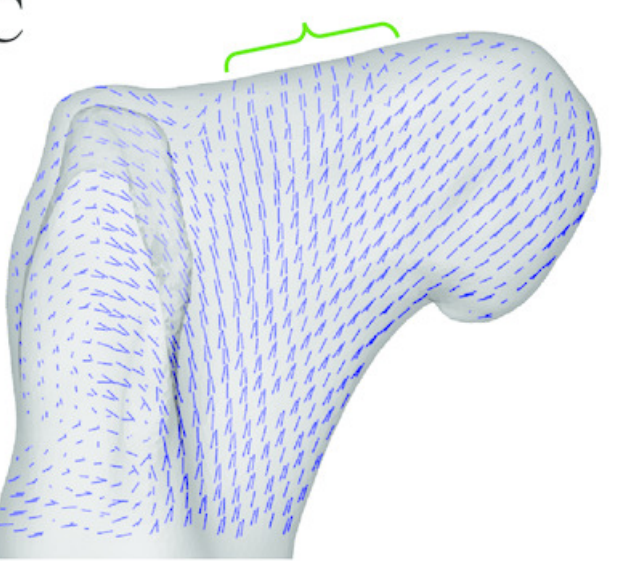

B

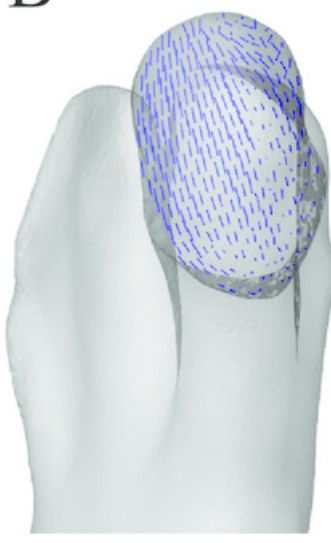

$\mathrm{D}$

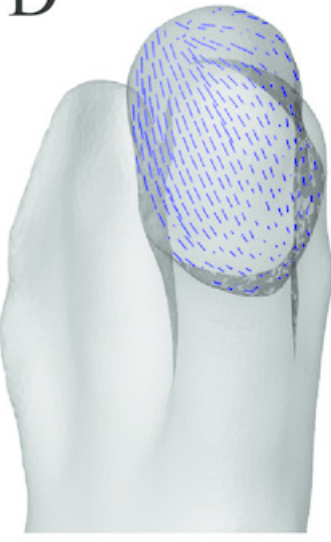




\section{Figure 13}

Comparison of parameters related to posture, extracted from the solution postures of the three species modelled: Daspletosaurus (' $\mathrm{D}$ '), ' Troodon ' (' $\mathrm{T}$ ') and the chicken ('C').

(A) Schematic illustration of the solution postures obtained for the three species, along with the location of the whole-body centre of mass (black and white disc). ( B) W hole-body centre of mass location anterior to the hips, normalized to total leg length. (C) Degree of crouch for each species, both as measured from the solution posture, as well as empirically predicted from the data reported by Bishop et al. (2018) . (D) Angles of the hip and knee joints. The hip extension angle is expressed relative to the horizontal, whereas the knee flexion angle is expressed relative to the femur. (E) Long-axis rotation and adduction-abduction of the hip joint. Positive values indicate external rotation and abduction (respectively), whereas negative values indicate internal rotation and adduction (respectively). 

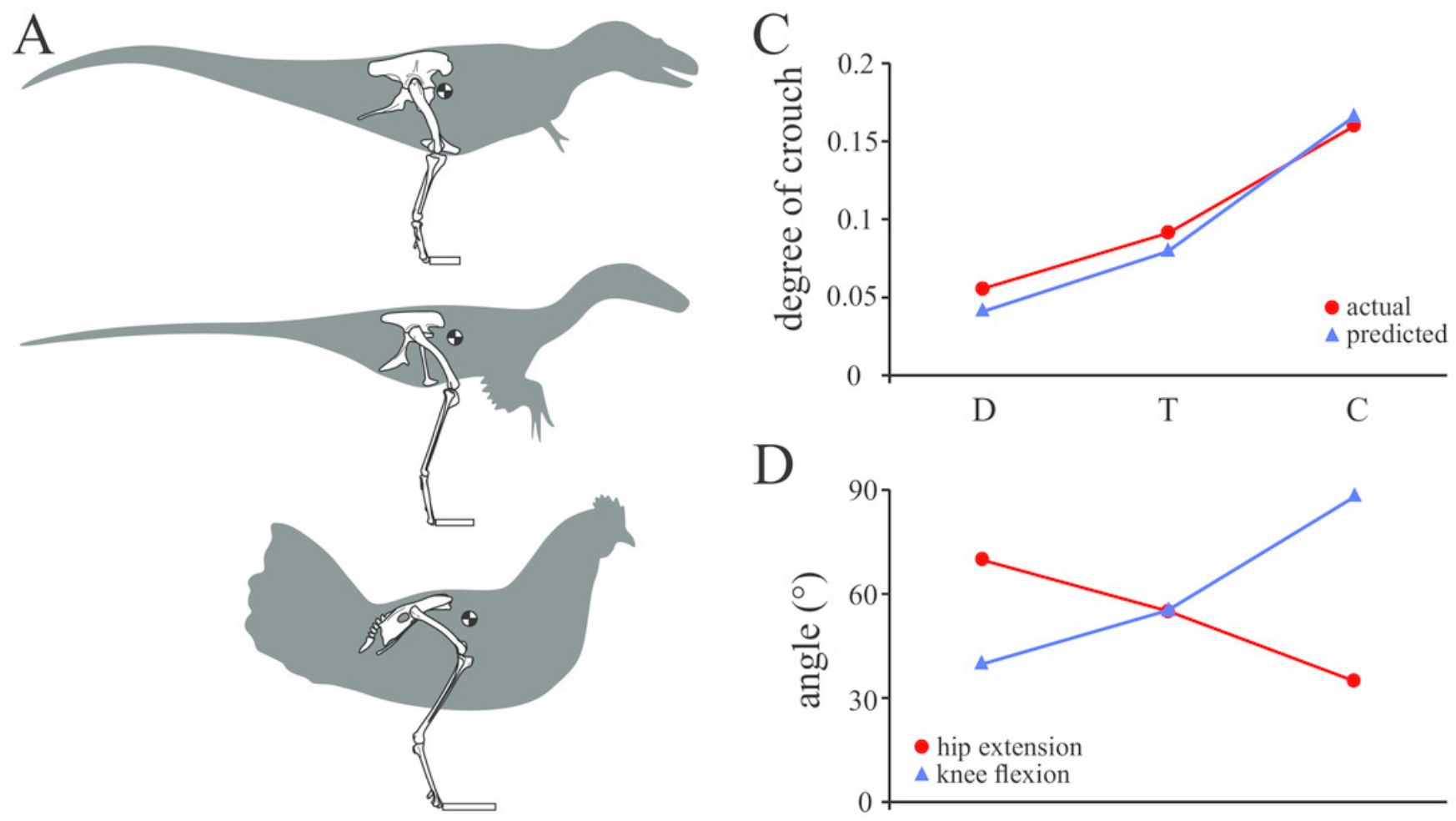

$\mathrm{D}$
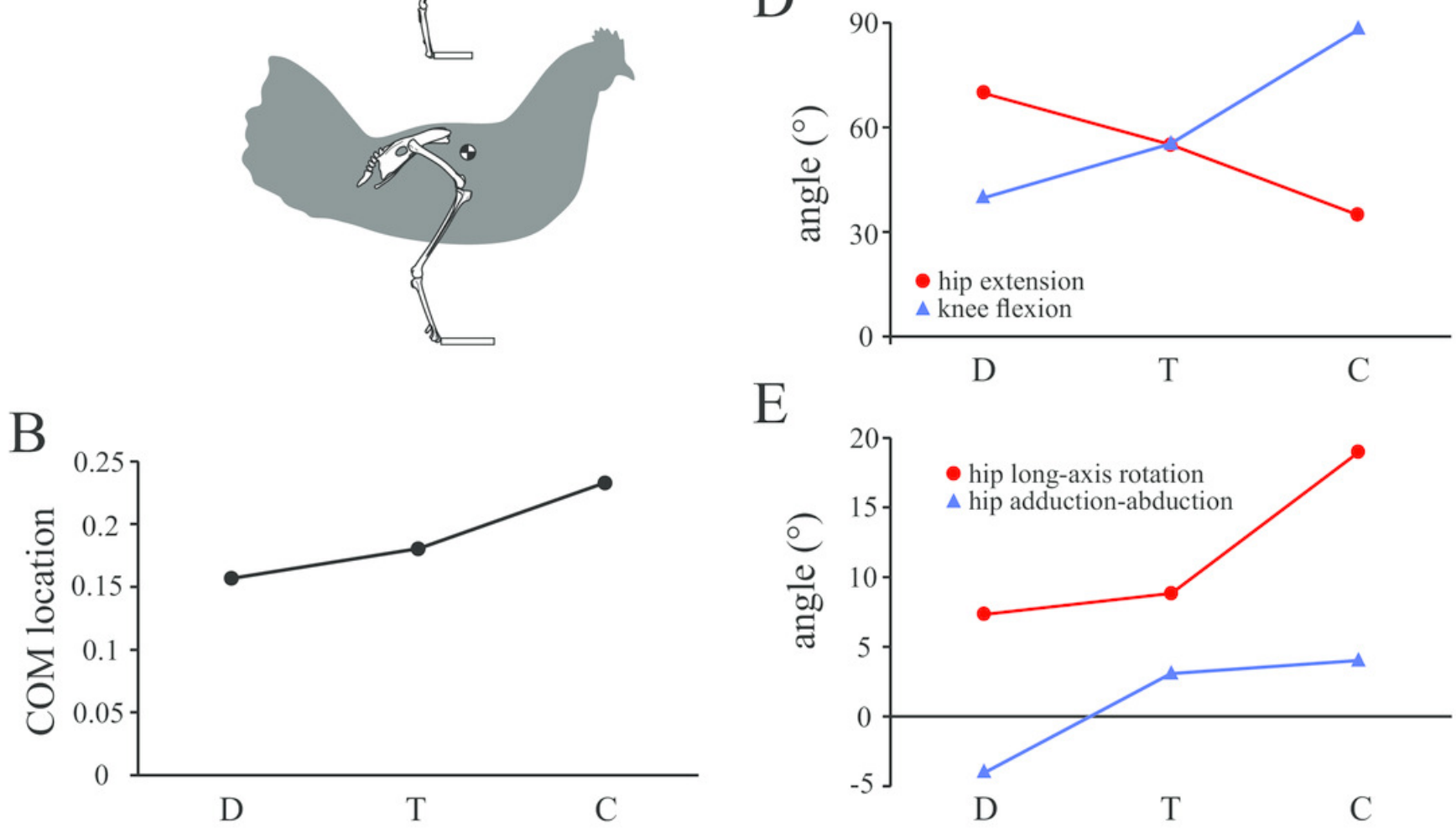

E

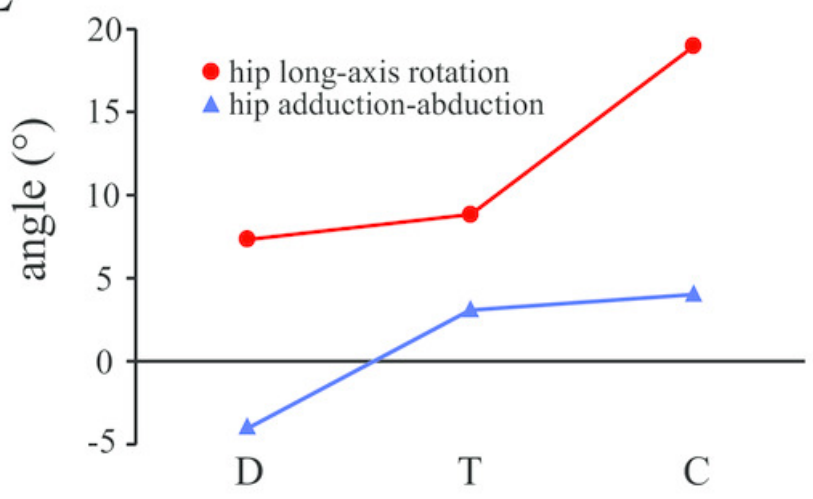




\section{Figure 14}

Comparison of parameters related to bone loading mechanics and muscular support, extracted from the solution postures of the three species modelled: Daspletosaurus ('D'), ' Troodon ' (' $T$ ') and the chicken (' $C$ ').

(A) Orientation of the neutral surface of bending and the orientation of principal stresses ( $\boldsymbol{\sigma}$ ${ }_{1}$ and $\boldsymbol{\sigma}_{3}$ ) relative to the femur long-axis, both measured at mid-shaft. Insets show the neutral surface with respect to the mid-shaft cross-section, as well as anatomical directions (' $A$ ', anterior; ' $P$ ', posterior; ' $M$ ', medial; ' $L$ ', lateral). (B) Ratio of maximum shear to bending stress in the femoral mid-shaft. (C) Normallized moments of hip abductor and medial rotator muscles. The hip abductor for all species is the iliofemoralis externus (activation set to zero in the chicken; see Part II). In Daspletosaurus and 'Troodon ', the medial rotators are the iliotrochantericus caudalis and puboischiofemorales internus 1 et 2 ; in the chicken, they are the iliotrochanterici caudalis et medius. (D) Oblique anterolateral view of the hip of Daspletosaurus, showing the abductor and medial rotator muscles (colours as in C). 
A

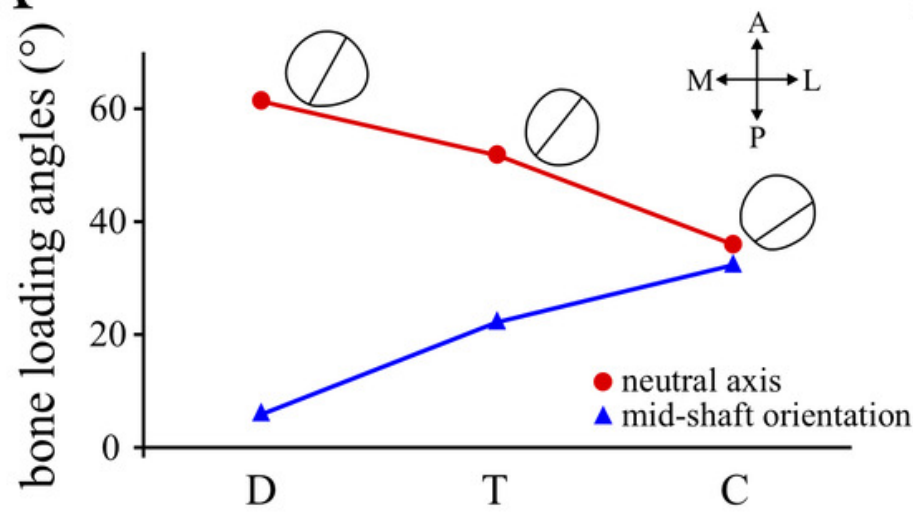

C

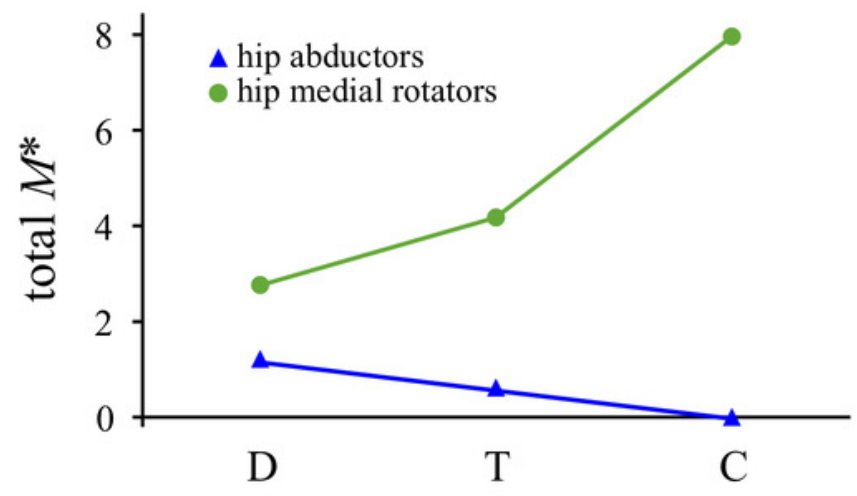

B

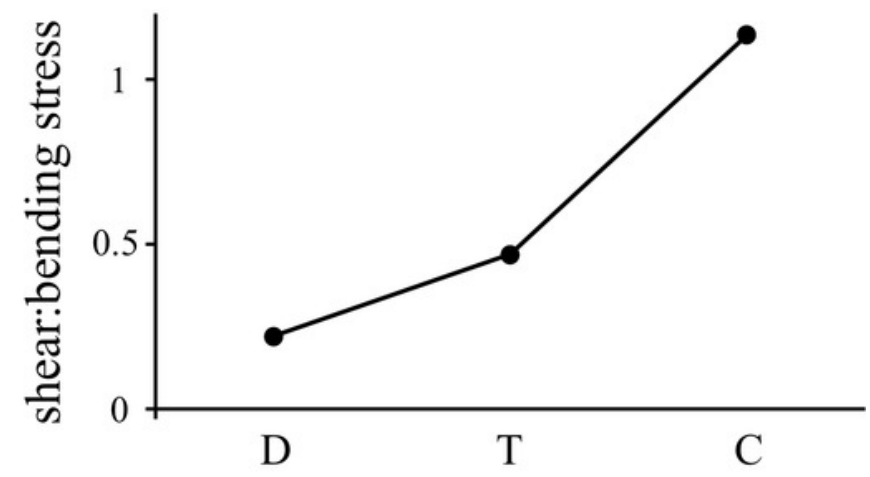

$\mathrm{D}$

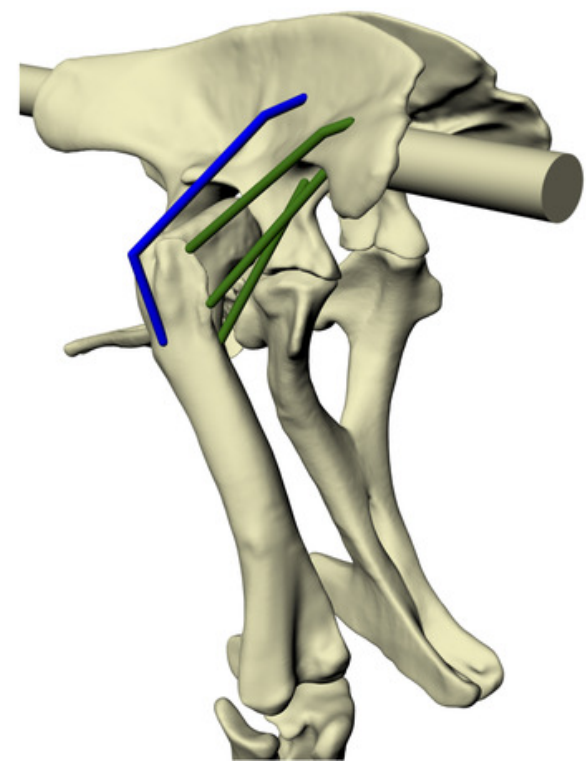

